\title{
Wegner Estimates and Localization for Gaussian Random Potentials
}

\author{
By \\ Naomasa UEKI*
}

\begin{abstract}
A Wegner estimate is proven for a Schrödinger operator with a bounded random vector potential and a Gaussian random scalar potential. The estimate is used to prove the strong dynamical localization and the exponential decay of the eigenfunctions. For the proof, Klopp's method using a vector field on a probability space and Germinet and Klein's bootstrap multiscale analysis are applied. Moreover Germinet and Klein's characterization of the Anderson metal-insulator transport transition is extended to the above operator.
\end{abstract}

\section{$\S 1 . \quad$ Introduction}

In this paper we give a Wegner estimate for a random Schrödinger operator

$$
\sum_{j=1}^{d}\left(i \partial_{j}+\mathcal{A}_{j}^{\omega}\right)^{2}+\mathcal{V}^{\omega}
$$

on $L^{2}\left(\mathbb{R}^{d}\right)$ and apply the estimate to study the spectral structure, where $i=$ $\sqrt{-1}, \partial_{j}=\partial /\left(\partial x_{j}\right)$ and $\left\{\left(\mathcal{A}_{1}^{\omega}(x), \ldots, \mathcal{A}_{d}^{\omega}(x), \mathcal{V}^{\omega}(x)\right), x \in \mathbb{R}^{d}, \omega \in \Omega\right\}$ are random fields on $\mathbb{R}^{d}$. For this subject, we have many works in the case that the vector potential $\mathcal{A}^{\omega}(x)=\left(\mathcal{A}_{1}^{\omega}(x), \ldots, \mathcal{A}_{d}^{\omega}(x)\right)$ is not random and the scalar potential $\mathcal{V}^{\omega}(x)$ is of the alloy-type: $\sum_{m \in \mathbb{Z}^{d}} \lambda_{m}^{\omega} u_{m}(x)$, where $\left\{\lambda_{m}^{\omega}, m \in \mathbb{Z}^{d}, \omega \in \Omega\right\}$

Communicated by Y. Takahashi. Received October 8, 2002.

2000 Mathematics Subject Classification(s): Primary, 82B44; Secondary 35J10, 35R60, $60 \mathrm{H} 25$

This work was supported by Grant-in-Aid for Scientific Research, The Ministry of Education, Sports, Science, Culture and Technology, Japan.

* Graduate School of Human and Environmental Studies, Kyoto University, Kyoto 6068501, Japan.

e-mail: ueki@math.h.kyoto-u.ac.jp 
is a family of independently and identically distributed real random variables and $u_{m}(x)$ are real deterministic functions on $\mathbb{R}^{d}[1],[5],[6],[7],[20],[22]$, [24], [25], [36], [39]. For the case that the vector potential $\mathcal{A}^{\omega}(x)$ is also random, Hislop and Klopp [20] recently give a Wegner estimate in the case that $\mathcal{A}_{j}^{\omega}(x), j=1, \ldots, d$, are alloy-type bounded random fields and $\mathcal{V}^{\omega}(x)=0$. More recently Klopp, Nakamura, Nakano and Nomura [23] showed Anderson localization for the corresponding discrete model. Apart from the alloy-type potential, Fischer, Hupfer, Leschke and Müller [11] and Hupfer, Leschke, Müller and Warzel [21] give a Wegner estimate for the case that the vector potential $\mathcal{A}^{\omega}(x)$ is not random and the scalar potential $\mathcal{V}^{\omega}(x)$ is a random field which is stationary in $x \in \mathbb{R}^{d}$. Their most fundamental example of the random field is a Gaussian random field. For other type of potentials, see [26]. The random potential $\left(\mathcal{A}^{\omega}(x), \mathcal{V}^{\omega}(x)\right)$ treated in this paper is a combination of a deterministic function and a Gaussian random field which is stationary in $x \in \mathbb{R}^{d}$. For this we extend Klopp's method [22] used in [20] so that his method can be applied to the Gaussian random potentials. The main idea is to represent the random field as a random Fourier series by referring the spectral representation (cf. [9]) of stationary random fields (see Lemma 2.2 below). This representation has the same form with the alloy-type potential such that the support of each single site potential $u_{m}(x)$ is noncompact. Because of technical reasons, we give only a weak form of the Wegner estimate only on a low energy interval under the condition that the covariance function of the Gaussian random field is compact. Under these restrictions, our estimate is a generalization of the estimates in [11] and [21] to the case that the vector potential and the scalar potential are correlated.

One difficulty of the random vector potential is same as that of the alloy type scalar potential $\sum_{m \in \mathbb{Z}^{d}} \lambda_{m}^{\omega} u_{m}(x)$ with nonsign definite single site potentials $\left\{u_{m}(x)\right\}_{m}$ treated in [6], [20], [22] and [39]: the quadratic form associated to the Schrödinger operator is not monotone as a function of random variables. Then a standard method extending a parameter to the complex space used in [5] and [27], which is used also for the Gaussian scalar potential in [11] and [21], seems to be not applicable. For many of such cases, Klopp's method [22] using a vector field on a probability space is the only effective method at present. This vector field is a number operator, whose eigenfunctions are homogeneous polynomials. Therefore his method is effective when the main part of the Schrödinger operator to give the Wegner estimate is a homogeneous polynomial on the probability space. In [20] Hislop and Klopp applies Klopp's method [22] to the random vector potential by using only the effect from the 
linear part $\sum_{j}\left(i \partial_{j} \mathcal{A}_{j}^{\omega}(x)+\mathcal{A}_{j}^{\omega}(x) i \partial_{j}\right)$ and neglecting the effect from the square part $\sum_{j}\left(\mathcal{A}_{j}^{\omega}(x)\right)^{2}$ under the condition that the vector potential $\mathcal{A}^{\omega}$ is small enough. The supremum norm of the vector potential $\mathcal{A}^{\omega}$ affects the energy interval where we can show the Wegner estimate. In this paper we assume that the vector potential is bounded and the scalar potential is the $n$-th power $W_{\omega}^{0}(x)^{n}$ of a Gaussian random field $W_{\omega}^{0}(x)$. We use only the effect from the scalar potential to give the result. As $n$ becomes larger, the effect from the scalar potential becomes stronger and the energy interval where we can show the Wegner estimate holds becomes wider (see (2.3) below). To clarify this situation, we use a Birman-Schwinger type operator whose parts of the square root are random (see (2.6) below). To treat this operator we need extra works not needed in [20]: we should consider the differentiation of the square root of the resolvent operator with respect to a parameter in the potential (see Lemma 2.3 below).

Our Wegner estimate can be used to prove the localization by the multiscale analysis. Since the pioneer work by Fröhlich and Spencer [13], this proof of localization has been extended, improved and simplified by many works. For this aspect, see [37] and the references therein. In particular Germinet and Klein give an effective multiscale analysis to deduce the strong dynamical localization from a weak initial estimate for a wide class of operators with short correlated potentials [14], [17]. In this paper we show that their methods are applicable to our case. The only task is to extend their theory to the operators unbounded below (see Corollary of Theorem 3.2 below). For this it is enough to use a control of Gaussian random fields in Lemma 5.3 in Fischer, Leschke and Müller [12] based on Fernique's theory [10].

Moreover Germinet and Klein [16], [18] recently show that the strong dynamical localization is equivalent with the initial estimate under some conditions. From this result, they show that some exponent $\beta^{-}(E)$, which is defined as a function of the energy parameter $E$ in (4.9) below, jumps from zero to a positive value as the energy parameter changes from an insulator region to a metallic region. Therefore this exponent gives a characterization of a transport property. In this paper we show that this theory is also extended to our case (see Theorem 4.1 and Proposition 4.3 below). For this we use again the estimate of the scalar potential based on Fernique's theory [10]. Since our Wegner estimate is weaker than the estimate used in [16], the lower bound of the size of the jump of the exponent $\beta^{-}(E)$ is different from that in [16]. We give a lower bound of the size in terms of the exponents appeared in the Wegner estimate (cf. Proposition 4.3 below). 
The organization of this paper is as follows. In Section 2 we give a Wegner estimate. In Section 3 we modify Germinet and Klein's theory on the multiscale analysis [14] to show the strong dynamical localization. In Section 4 we modify Germinet and Klein's theory on a characterization of the transport transition [16] so that their theory can be applied to the operators in this paper.

\section{$\S 2 . \quad$ Wegner Estimate}

\section{§2.1. Main theorem}

Let $\left\{\left(W_{\omega}^{j}(x)\right)_{j=0,1, \ldots, g}, x \in \mathbb{R}^{d}, \omega \in \Omega\right\}$ be an $\mathbb{R}^{g+1}$-valued stationary ergodic Gaussian random field with mean zero and the covariance $R^{j k}(x)=$ $E\left[W_{\omega}^{j}(x) W_{\omega}^{k}(0)\right]$ represented as

$$
R^{j k}(x)=\sum_{\iota=1}^{r} \int \sigma_{\iota}^{j}(x+y) \overline{\sigma_{\iota}^{k}(y)} d y
$$

for some complex valued $C^{s}$ functions $\sigma_{\iota}^{j}(x), 0 \leq j \leq g, 1 \leq \iota \leq r$, on $\mathbb{R}^{d}$ with compact support and $s \geq d+4$. Let $A(x, w)$ be an $\mathbb{R}^{d}$ valued $C^{1}$ function of $(x, w) \in \mathbb{R}^{d} \times \mathbb{R}^{g}$ such that $\nabla_{w} A(x, w)$ is a $C^{2}$ function of $(x, w)$ and $w \cdot \nabla_{w} A$ and $\nabla_{w} A$ are bounded. For each $L>0$, let $\Lambda_{L}:=(-L / 2, L / 2)^{d}$ be an open box and, for $L>3$, let $\widetilde{\chi_{L}}$ be a smooth function on $\mathbb{R}^{d}$ such that $0 \leq \widetilde{\chi_{L}} \leq 1$, $\widetilde{\chi_{L}}=1$ on $\Lambda_{L-3}, \widetilde{\chi_{L}}=0$ on $\Lambda_{L-1}^{c}$ and $\left|\nabla \widetilde{\chi_{L}}\right| \leq 2$. We consider a self-adjoint operator on $L^{2}\left(\mathbb{R}^{d}\right)$ defined by

$$
\widetilde{H_{L}^{\omega}}:=\sum_{j=1}^{d}\left(i \partial_{j}+\widetilde{\chi_{L}} A_{j}\left(x, \overline{W_{\omega}}(x)\right)\right)^{2}-\widetilde{\chi_{L}} W_{\omega}^{0}(x)^{n},
$$

where $\overline{W_{\omega}}(x)={ }^{t}\left(W_{\omega}^{1}(x), \ldots, W_{\omega}^{g}(x)\right)$. If we define a positive operator by

$$
\widehat{H_{L}^{\omega}}:=\sum_{j=1}^{d}\left(i \partial_{j}+\widetilde{\chi_{L}} A_{j}\left(x, \overline{W_{\omega}}(x)\right)\right)^{2}
$$

then we see that $\widetilde{\chi_{L}} W_{\omega}^{0}(x)^{n}$ is $\widehat{H_{L}^{\omega}}$-compact. Therefore the spectrum in $(-\infty, 0)$ of the operator $\widetilde{H_{L}^{\omega}}$ consists only of its discrete spectrum. For the spectrum, we will prove the following Wegner estimate in this section:

Theorem 2.1. For any

$$
E_{0}<-\left(2\left\|w \cdot \nabla_{w} A\right\|_{\infty} / n\right)^{2},
$$


there exists a finite constant $Q\left(E_{0}\right)$ such that

$$
P\left(\operatorname{dist}\left(E, \sigma\left(\widetilde{H_{L}^{\omega}}\right)\right) \leq \eta\right) \leq Q\left(E_{0}\right) \eta^{1-d / s} L^{d(2-d /(2 s))}
$$

for any $E \leq E_{0}, L \geq 4$ and $\eta>0$, where, for any self-adjoint operator $A, \sigma(A)$ is the spectral set of $A$.

This estimate can be used to show results on the spectral localization of the self-adjoint operator

$$
H^{\omega}:=\sum_{j=1}^{d}\left(i \partial_{j}+A_{j}\left(x, \overline{W_{\omega}}(x)\right)\right)^{2}-W_{\omega}^{0}(x)^{n},
$$

on $L^{2}\left(\mathbb{R}^{d}\right)$. This is the subject of the next section.

Remark 2.1. In the case that $A$ is independent of $w$ and $n=1$, Fisher, Hupfer, Leschke and Müller [11] and Hupfer, Leschke, Müller and Warzel [21] give a strong form of the Wegner estimate under more general conditions, where the compactness of the covariance function is not assumed. Their estimate is as follows: let $H_{L, X}^{\omega}$ be the restriction of the operator $\sum_{j=1}^{d}\left(i \partial_{j}+A_{j}(x)\right)^{2}-W_{\omega}^{0}(x)$ to $L^{2}\left(\Lambda_{L}\right)$ by the Dirichlet or Neumann boundary conditions. Then there exists an explicitly written function $C_{W}(E)$ such that

$$
E\left[\sharp\left(\sigma\left(H_{L, X}^{\omega}\right) \cap[E-\eta, E+\eta]\right)\right] \leq C_{W}(E+\eta) \eta L^{d}
$$

for all $E \in \mathbb{R}$ and $\eta \geq 0$. From this estimate, we see that the function $C_{W}(E)$ is an upper bound of the density of states. This estimate is the first motivation of Wegner's estimate [40].

Remark 2.2. $\quad$ (i) Theorem 2.1 is extended to the operator

$$
\widetilde{H_{L, 1}^{\omega}}:=\widetilde{H_{L}^{\omega}}+\widetilde{\chi_{L}} V\left(x, \overline{W_{\omega}}(x)\right)
$$

where $V(x, w)$ be a real bounded continuous function of $(x, w) \in \mathbb{R}^{d} \times \mathbb{R}^{g}$ such that $V(x, w)$ is $C^{1}$ in $w$ and $\nabla_{w} V$ and $w \cdot \nabla_{w} V$ are bounded. For this operator, (2.4) holds only for $E_{0}<\mathfrak{E}_{0}$, where

$$
\mathfrak{E}_{0}:=\left\{\begin{array}{l}
-(2 \mathfrak{A} / n)^{2} \text { if } \mathfrak{V} \leq 0, \\
-\left\{\mathfrak{V} /\left(\sqrt{\mathfrak{A}^{2}+n \mathfrak{V}}-\mathfrak{A}\right)\right\}^{2} \text { if } \mathfrak{V}>0,
\end{array}\right.
$$

$\mathfrak{A}:=\left\|w \cdot \nabla_{w} A\right\|_{\infty}$ and $\mathfrak{V}:=\sup \left(w \cdot \nabla_{w} V-n V\right)$. 
(ii) Theorem 2.1 is extended to the operator

$$
\widetilde{H_{L, 2}^{\omega}}:=\widehat{H_{L}^{\omega}}+\widetilde{\chi_{L}} p_{n}\left(\overline{W_{\omega}^{0}}(x)\right)
$$

without changing the statement, where $p_{n}\left(t_{1}, \ldots, t_{h}\right)$ is a real homogeneous polynomial of degree $n$ and $\left\{\overline{W_{\omega}^{0}}=\left(W_{\omega}^{0, k}(x)\right)_{k=1,2, \ldots, h}: x \in \mathbb{R}^{d}, \omega \in \Omega\right\}$ is an $\mathbb{R}^{h}$ valued random field such that $\left\{\left(\overline{W_{\omega}^{0}}(x), \overline{W_{\omega}}(x)\right): x \in \mathbb{R}^{d}, \omega \in \Omega\right\}$ is an $\mathbb{R}^{g+h}$ valued random field satisfying the same conditions for the random field $\left\{\left(W_{\omega}^{0}(x), \overline{W_{\omega}}(x)\right): x \in \mathbb{R}^{d}, \omega \in \Omega\right\}$ stated at the front of this section.

(iii) Theorem 2.1 is extended to the operator

$$
\widetilde{H_{L, 3}^{\omega}}:=\widehat{H_{L}^{\omega}}-\widetilde{\chi_{L}} q_{n}\left(W_{\omega}^{0}(x)\right)
$$

without changing the statement, where $q_{n}(t)$ is a polynomial such that $t q_{n}^{\prime}(t) \geq$ $n q_{n}(t)$. For example, we can take as $q_{2 m-1}(t)=t^{2 m}+a t^{2 m-1}$.

\section{§2.2. Proof of the Wegner estimate}

For the proof of Theorem 2.1, we introduce a Birman-Schwinger type operator by

$$
\Gamma(\omega, L, E)=\left(\widehat{H_{L}^{\omega}}-E\right)^{-1 / 2} \widetilde{\chi_{L}} W_{\omega}^{0}(x)^{n}\left(\widehat{H_{L}^{\omega}}-E\right)^{-1 / 2}
$$

It is a different point from [6], [20] and [22] that the operator $\left(\widehat{H_{L}^{\omega}}-E\right)^{-1 / 2}$ depends on $\omega$. We deduce $(2.4)$ only by the effect from the part $\widetilde{\chi_{L}} W_{\omega}^{0}(x)^{n}$.

For any $p>0$, let $\mathcal{I}_{p}$ be the trace or super-trace class: a continuous linear operator $A$ on $L^{2}\left(\mathbb{R}^{d}\right)$ is said to belong to $\mathcal{I}_{p}$ if $\||A|||_{p}:=\left(\operatorname{Tr}\left[|A|^{p}\right]\right)^{1 / p}<\infty$, where $|A|:=\left(A^{*} A\right)^{1 / 2}$ (cf. [6], [7], [20], [33]). We use $\|\cdot\|_{p}$ for the norm on $L^{p}\left(\mathbb{R}^{d}\right)$ and ||$\cdot \mid \|$ for the operator norm.

Then we have the following:

Lemma 2.1. Let $q \geq \min \{q \in 4 \mathbb{N}: q>d\}$. Let $A \in L_{\text {loc }}^{4}\left(\mathbb{R}^{d} \rightarrow \mathbb{R}^{d}\right)$ such that $\nabla \cdot A \in L_{\text {loc }}^{2}\left(\mathbb{R}^{d}\right)$. We set $H(A)=\sum_{j=1}^{d}\left(i \partial_{j}+A_{j}\right)^{2}$. Then we have the following:

(i) There exists a finite constant $c_{1}$ depending only on $q$ and $d$ such that

$$
\left\|\left|(H(A)-E)^{-1 / 2} k\right|\right\|_{q} \leq c_{1}\|k\|_{q} /|E|^{(q-d) /(2 q)}
$$

for any $E<0$ and $k \in L^{q}\left(\mathbb{R}^{d}\right)$. 
(ii) There exists a finite constant $c_{2}$ depending only on $q$ and $d$ such that

$$
\left\|\left|(H(A)-E)^{-1 / 2} k(H(A)-E)^{-1 / 2}\right|\right\|_{q / 2} \leq c_{2}\|k\|_{q / 2} /|E|^{(q-d) / q}
$$

for any $E<0$ and $k \in L^{q / 2}\left(\mathbb{R}^{d}\right)$.

(iii) There exists a finite constant $c_{3}$ depending only on $q$ and $d$ such that

$$
\left\|\left.\left|(H(A)-E)^{-1 / 2} h \cdot(i \nabla+A)(H(A)-E)^{-1 / 2}\|\|_{q} \leq c_{3}\|h\|_{q} /\right| E\right|^{(q-d) /(2 q)}\right.
$$

for any $E<0$ and $\mathbb{C}^{d}$ valued $L^{q}$ function $h$ on $\mathbb{R}^{d}$.

(iv) There exists a finite constant $c_{4}$ depending only on $q$ and $d$ such that

$$
\left\|\left|(H(A)-E)^{-1} k\right|\right\|_{q / 2} \leq c_{4}\|k\|_{q / 2} /|E|^{(q-d) / q}
$$

for any $E<0$ and $k \in L^{q / 2}\left(\mathbb{R}^{d}\right)$.

Proof. (i) By the diamagnetic inequality, we have

$$
\left|(H(A)-E)^{-1 / 2} \varphi\right| \leq(H(0)-E)^{-1 / 2}|\varphi|
$$

for any $\varphi \in C_{0}^{\infty}\left(\mathbb{R}^{d}\right)$ (see (4.9) in [29] and (A.23) in [21]). We now assume that $q \in 2 \mathbb{N}$. By using (2.7) successively, we have

$$
\|\left.(H(A)-E)^{-1 / 2} k\right|^{q} \varphi|\leq|(H(0)-E)^{-1 / 2}|k|^{q}|\varphi|,
$$

where, for any operator $L,|L|:=\sqrt{L^{*} L}$. From this, Lemma 15.11 in [34] and Theorem 4.1 in [33], we have

$$
\left\|\left|(H(A)-E)^{-1 / 2} k\|\|_{q} \leq\right|\right\|(H(0)-E)^{-1 / 2}|k|\|\|_{q} \leq c_{1}\|k\|_{q} /|E|^{(q-d) /(2 q)} .
$$

For $q \notin 2 \mathbb{N}$, we use the Stein interpolation theorem (cf. [32] Theorem IX.21).

(ii) Since

$$
\begin{aligned}
& \|\left.\left|(H(A)-E)^{-1 / 2} k(H(A)-E)^{-1 / 2}\right|\right|_{q / 2} \\
& \leq\left\|\left|(H(A)-E)^{-1 / 2} \sqrt{|k|}\right|||_{q}||\left|\sqrt{|k|}(H(A)-E)^{-1 / 2}\right|\right\|_{q},
\end{aligned}
$$

(i) implies (ii).

(iii) Since

$$
\left\|\left(i \partial_{j}+A_{j}\right)(H(A)-E)^{-1 / 2}\right\|^{2} \leq 1
$$

for any $j$, we have

$$
\begin{aligned}
& \|\|(H(A)-E)^{-1 / 2} h \cdot(i \nabla+A)(H(A)-E)^{-1 / 2} \|_{q} \\
& \leq \sum_{j=1}^{d}\left\|(H(A)-E)^{-1 / 2} h_{j}\right\| \|_{q} .
\end{aligned}
$$


Therefore (i) implies (iii).

(iv) For $q \in 4 \mathbb{N}$, as in (i), we have

$$
\|\left.\left.(H(A)-E)^{-1} k\right|^{q / 2} \varphi|\leq|(H(0)-E)^{-1}|k|\right|^{q / 2}|\varphi|
$$

and

$$
\left\|\left|(H(A)-E)^{-1 / 2} k\right|\right\|_{q / 2} \leq\left.\left|\left\|(H(0)-E)^{-1 / 2}|k|\right\|\left\|_{q / 2} \leq c_{4}\right\| k \|_{q / 2} /\right| E\right|^{(q-d) / q} .
$$

For $q \notin 4 \mathbb{N}$, we use the interpolation theorem.

In the following we fix $q \in \mathbb{N}$ such that $q \geq \min \{q \in 4 \mathbb{N}: q>d\}$. Lemma 2.1 (ii) implies $\Gamma(\omega, L, E) \in \mathcal{I}_{q / 2}$. We take a smooth function $\rho$ having the form $\rho=\sigma^{q}$, where $\sigma$ is a smooth function on $\mathbb{R}$ such that $0 \leq \sigma \leq 1$ on $\mathbb{R}, \sigma=1$ on $(-\infty,-1 / 2]$ and $\sigma=0$ on $[1 / 2, \infty)$. For any self-adjoint operator $A$, let $E(\cdot ; A)$ be the spectral projection of $A$. As in [6], [20] and [22], we have

$$
\begin{aligned}
& P\left(\operatorname{dist}\left(E, \sigma\left(\widetilde{H_{L}^{\omega}}\right)\right) \leq \eta\right) \\
& \leq E[\operatorname{Tr}[E([1-\kappa, 1+\kappa]: \Gamma(\omega, L, E))]] \\
& \leq E\left[\operatorname{Tr}\left[\rho_{\kappa}(\Gamma(\omega, L, E)-1-3 \kappa / 2)-\rho_{\kappa}(\Gamma(\omega, L, E)-1+3 \kappa / 2)\right]\right] \\
& =\int_{-3 \kappa / 2}^{3 \kappa / 2} d t E\left[\operatorname{Tr}\left[\left(-\rho_{\kappa}\right)^{\prime}(\Gamma(\omega, L, E)-1+t)\right]\right]
\end{aligned}
$$

where $\kappa=\eta /|E|$ and $\rho_{\kappa}(\mu)=\rho(\mu / \kappa)$.

To consider an associated vector field used in [6], [20] and [22], we refer the theory of the spectral representation of stationary field (cf. [9]) to give the following representation:

Lemma 2.2. The random field $W_{\omega}(x)=\left(W_{\omega}^{j}(x)\right)_{j=0}^{g}$ is represented as

$$
W_{\omega}^{j}(x)=\sum_{\iota=1}^{2 r} \sum_{m \in \mathbb{Z}^{d}} W_{\omega}^{\iota, m} e_{\iota, m}^{j}(x)
$$

in $L^{p}\left(\Lambda_{L} \times \Omega\right)$ for any $1 \leq p<\infty$, where $\left\{W_{\omega}^{\iota, m}\right\}_{1 \leq \iota \leq 2 r, m \in \mathbb{Z}^{d}}$ is a family of independently and identically distributed random variables with the standard normal distribution and $e_{\iota, m}^{j}, 1 \leq \iota \leq 2 r, m \in \mathbb{Z}^{d}, 0 \leq j \leq g$, are $C^{s}$ functions such that, for any $0 \leq \ell \leq s$,

$$
\sup _{x \in \Lambda_{L}}\left|\nabla^{\ell} e_{\iota, m}^{j}(x)\right| \leq c L^{s-\ell-d / 2}|m|_{\infty}^{\ell-s}
$$

where $c$ is some finite constant depending only on the covariance $R=\left(R^{j k}(x)\right)$. 
Proof. Let $\left\{W_{\iota}(h): h \in L^{2}\left(\mathbb{R}^{d}\right)\right\}_{1 \leq \iota \leq 2 r}$ be $2 r$ independent copies of the isonormal Gaussian random field: for each $h_{1}, h_{2}, \ldots, h_{m} \in L^{2}\left(\mathbb{R}^{d}\right), W_{\iota}\left(h_{1}\right)$, $W_{\iota}\left(h_{2}\right), \ldots, W_{\iota}\left(h_{m}\right)$ are Gaussian random variables with mean zero and

$$
E\left[W_{\iota}\left(h_{1}\right) W_{\iota}\left(h_{2}\right)\right]=\int_{\mathbb{R}^{d}} h_{1}(\xi) h_{2}(\xi) d \xi
$$

(cf. Definition 1.1.1 in Nualart [30]). We define the Fourier transform of $f \in$ $L^{1}\left(\mathbb{R}^{d}\right)$ by

$$
\widehat{f}(\xi)=\int_{\mathbb{R}^{d}} e^{-2 \pi i \xi \cdot x} f(x) d x .
$$

Then the random field $W_{\omega}(x)$ has the following representation:

$$
W_{\omega}^{j}(x)=\sum_{\iota=1}^{r}\left\{W_{\iota}\left(\operatorname{Re}\left(e^{2 \pi i \xi \cdot x} \widehat{\sigma_{\iota}^{j}}(\xi)\right)\right)+W_{r+\iota}\left(\operatorname{Im}\left(e^{2 \pi i \xi \cdot x} \widehat{\sigma_{\iota}^{j}}(\xi)\right)\right)\right\} .
$$

We here note that

$$
e^{2 \pi i \xi \cdot x} \widehat{\sigma_{\iota}^{j}}(\xi)=\widehat{\sigma_{\iota}(\cdot+x)}(\xi) .
$$

If $\operatorname{supp} \sigma_{\iota}^{j} \subset \Lambda_{R}$ and $x \in \Lambda_{L}$, then $\operatorname{supp} \sigma_{\iota}^{j}(\cdot+x) \subset \Lambda_{R+L}$. Then we take a complete orthonormal basis $\left\{h_{m}(y):=\prod_{k=1}^{d} k_{m_{k}}\left(y_{k}\right): m \in \mathbb{Z}^{d}\right\}$ of $L^{2}\left(\Lambda_{R+L}\right)$, where $y=\left(y_{1}, y_{2}, \ldots, y_{d}\right), k_{0}\left(y_{1}\right)=1 / \sqrt{R+L}$, and, for $m_{1}>0$,

$$
k_{-m_{1}}\left(y_{1}\right)=\sqrt{\frac{2}{R+L}} \sin \frac{2 m_{1} \pi y_{1}}{R+L} \quad \text { and } \quad k_{m_{1}}\left(y_{1}\right)=\sqrt{\frac{2}{R+L}} \cos \frac{2 m_{1} \pi y_{1}}{R+L} .
$$

We extend these functions to $\mathbb{R}^{d}$ by $h_{m}(y)=0$ on $\Lambda_{R+L}^{c}$. Then, for each $x \in \Lambda_{L}$, we have

$$
\sigma_{\iota}^{j}(y+x)=\sum_{m \in \mathbb{Z}^{d}} h_{m}(y) \int h_{m}(z) \sigma_{\iota}^{j}(z+x) d z
$$

and

$$
e^{2 \pi i \xi \cdot x} \widehat{\sigma_{\iota}^{j}}(\xi)=\sum_{m \in \mathbb{Z}^{d}} \widehat{h_{m}}(\xi) \int h_{m}(y) \sigma_{\iota}^{j}(y+x) d y
$$

in $L^{2}\left(\mathbb{R}^{d}\right)$. Let $\left(\mathbb{Z}^{d}\right)_{e}:=\left\{m=\left(m_{1}, m_{2}, \ldots, m_{d}\right) \in \mathbb{Z}^{d}: \sharp\left\{j: m_{j}<0\right\}\right.$ is even $\}$ and $\left(\mathbb{Z}^{d}\right)_{o}:=\left\{m=\left(m_{1}, m_{2}, \ldots, m_{d}\right) \in \mathbb{Z}^{d}: \sharp\left\{j: m_{j}<0\right\}\right.$ is odd $\}$. Then $\widehat{h_{m}}(\xi)$ is real valued for $m \in\left(\mathbb{Z}^{d}\right)_{e}$ and is purely imaginary valued for $m \in\left(\mathbb{Z}^{d}\right)_{o}$. Thus we have

$$
\operatorname{Re}\left(e^{2 \pi i \xi \cdot x} \widehat{\sigma_{\iota}^{j}}(\xi)\right)=\sum_{m \in\left(\mathbb{Z}^{d}\right)_{e}} \widehat{h_{m}}(\xi) e_{\iota, m}^{j}(x)+\sum_{m \in\left(\mathbb{Z}^{d}\right)_{o}} i \widehat{h_{m}}(\xi) e_{\iota, m}^{j}(x),
$$


where

$$
e_{\iota, m}^{j}(x):=\left\{\begin{array}{l}
\int h_{m}(y) \operatorname{Re} \sigma_{\iota}^{j}(y+x) d y \text { for } m \in\left(\mathbb{Z}^{d}\right)_{e}, \\
\int h_{m}(y) \operatorname{Im} \sigma_{\iota}^{j}(y+x) d y \text { for } m \in\left(\mathbb{Z}^{d}\right)_{o} .
\end{array}\right.
$$

Similarly we have

$$
\operatorname{Im}\left(e^{2 \pi i \xi \cdot x} \widehat{\sigma_{\iota}^{j}}(\xi)\right)=\sum_{m \in\left(\mathbb{Z}^{d}\right)_{e}} \widehat{h_{m}}(\xi) e_{r+\iota, m}^{j}(x)-\sum_{m \in\left(\mathbb{Z}^{d}\right)_{o}} i \widehat{h_{m}}(\xi) e_{r+\iota, m}^{j}(x),
$$

where

$$
e_{r+\iota, m}^{j}(x):=\left\{\begin{array}{l}
\int h_{m}(y) \operatorname{Im} \sigma_{\iota}^{j}(y+x) d y \text { for } m \in\left(\mathbb{Z}^{d}\right)_{e}, \\
\int h_{m}(y) \operatorname{Re} \sigma_{\iota}^{j}(y+x) d y \text { for } m \in\left(\mathbb{Z}^{d}\right)_{o} .
\end{array}\right.
$$

Then we have (2.8) for each $x \in \Lambda_{L}$ in $L^{2}(\Omega)$, where

$$
W_{\omega}^{\iota, m}:=\left\{\begin{array}{l}
W_{\iota}\left(\widehat{h_{m}}\right) \text { for } m \in\left(\mathbb{Z}^{d}\right)_{e} \\
W_{\iota}\left(i \widehat{h_{m}}\right) \text { for } m \in\left(\mathbb{Z}^{d}\right)_{o}
\end{array}\right.
$$

and

$$
W_{\omega}^{r+\iota, m}:=\left\{\begin{array}{l}
W_{r+\iota}\left(\widehat{h_{m}}\right) \text { for } m \in\left(\mathbb{Z}^{d}\right)_{e} \\
W_{r+\iota}\left(-i \widehat{h_{m}}\right) \text { for } m \in\left(\mathbb{Z}^{d}\right)_{o} .
\end{array}\right.
$$

By the integration by parts, for each $0 \neq m \in \mathbb{Z}^{d}$ and $0 \leq \ell<s$, there exist $m^{\prime} \in \mathbb{Z}^{d}$ and $k \in\{1,2, \ldots, d\}$, such that

$$
\begin{aligned}
& \left|\int h_{m}(y) \nabla^{\ell} \sigma_{\iota}^{j}(y+x) d y\right| \\
& =\left(\frac{R+L}{2 \pi|m|_{\infty}}\right)^{s-\ell}\left|\int h_{m^{\prime}}(y)\left(\frac{\partial}{\partial y^{k}}\right)^{s-\ell} \nabla^{\ell} \sigma_{\iota}^{j}(y+x) d y\right|,
\end{aligned}
$$

where $|m|_{\infty}$ is the maximum norm on $\mathbb{Z}^{d}$. Since $\left|h_{m^{\prime}}(y)\right| \leq \sqrt{2 /(R+L)}^{d}$, we obtain (2.9). Then we see that $\left\{\sum_{\iota=1}^{2 r} \sum_{|m|_{\infty} \leq N} W_{\omega}^{\iota, m} e_{\iota, m}^{j}\right\}_{N}$ is a Cauchy sequence in $L^{p}\left(\Lambda_{L} \times \Omega\right)$. Therefore (2.8) holds in $L^{p}\left(\Lambda_{L} \times \Omega\right)$.

Remark 2.3. If $0 \leq \ell<s-d$, we see that $\left\{\sum_{\iota=1}^{2 r} \sum_{|m|_{\infty} \leq N} W_{\omega}^{\iota, m}\right.$ $\left.\nabla^{\ell} e_{\iota, m}^{j}\right\}_{N}$ is a Cauchy sequence in $L^{p}\left(\Lambda_{L} \times \Omega\right)$ by (2.9). Therefore, for each $k \in \mathbb{Z}_{+}$, if $s \geq d+2+k$, then we see that $\left\{\sum_{\iota=1}^{2 r} \sum_{|m|_{\infty} \leq N} W_{\omega}^{\iota, m} \nabla^{k} e_{\iota, m}^{j}(x)\right\}_{N}$ converges to $\nabla^{k} W_{\omega}^{j}(x)$ uniformly on $\Lambda_{L}$ and $W_{\omega}^{j}(x)$ is a $C^{k}$ function of $x$ almost surely. Since we assume $s \geq d+4, W_{\omega}^{j}(x)$ is a $C^{2}$ function of $x$. 
We use the following lemma on differentiations in random variables:

Lemma 2.3. For any $\varepsilon>0,1 \leq \iota \leq 2 r$ and $m \in \mathbb{Z}^{d}$, we set

$$
\begin{aligned}
W_{\omega}^{j}(x, \varepsilon ; \iota, m) & :=W_{\omega}^{j}(x)+\varepsilon e_{\iota, m}^{j}(x), \\
\widehat{H_{L}^{\omega}}(\varepsilon ; \iota, m) & :=\sum_{j=1}^{d}\left(i \partial_{j}+\widetilde{\chi_{L}} A_{j}\left(x, \overline{W_{\omega}}(x, \varepsilon ; \iota, m)\right)\right)^{2}
\end{aligned}
$$

and

$$
\begin{aligned}
\Gamma(\omega, L, E, \varepsilon ; \iota, m):=\left(\widehat{H_{L}^{\omega}}(\varepsilon ; \iota, m)-E\right)^{-1 / 2} \widetilde{\chi_{L}} W_{\omega}^{0}(x, \varepsilon ; \iota, m)^{n} \\
\times\left(\widehat{H_{L}^{\omega}}(\varepsilon ; \iota, m)-E\right)^{-1 / 2} .
\end{aligned}
$$

Then we have the following:

(i) In the weak sense, we have

$$
\left\{\frac{\partial}{\partial \varepsilon}\left(\widehat{H_{L}^{\omega}}(\varepsilon ; \iota, m)-E\right)^{-1 / 2}\right\}_{\varepsilon=0}=\Xi(\omega, L, E ; \iota, m),
$$

where $\Xi(\omega, L, E ; \iota, m)$ is a bounded operator defined by the following integral in the weak sense:

$$
\begin{aligned}
& \Xi(\omega, L, E ; \iota, m):=\frac{-1}{\sqrt{\pi}} \int_{0}^{\infty} d t \sqrt{t} e^{t E} \int_{0}^{1} d u \exp \left(-u t \widehat{H_{L}^{\omega}}\right) \\
& \quad \times \sum_{j=1}^{d}\left[\widetilde{\chi_{L}} \overline{e_{\iota, m}}(x) \cdot \nabla_{w} A_{j}\left(x, \overline{W_{\omega}}(x)\right), i \partial_{j}+\widetilde{\chi_{L}} A_{j}\left(x, \overline{W_{\omega}}(x)\right)\right]_{+} \\
& \quad \times \exp \left(-(1-u) t \widehat{t H_{L}^{\omega}}\right),
\end{aligned}
$$

where $\overline{e_{\iota, m}}(x):={ }^{t}\left(e_{\iota, m}^{1}(x), \ldots, e_{\iota, m}^{g}(x)\right)$ and $[A, B]_{+}:=A B+B A$ for any operators $A$ and $B$.

(ii) The operator $\Xi(\omega, L, E ; \iota, m)$ defined in (i) maps the elements of $\bigcup_{a>0} \operatorname{Ran} E([a, \infty): \Gamma(\omega, L, E))$ to the domain of $\widehat{H_{L}^{\omega}}$, where, for any opera$\operatorname{tor} A, \operatorname{Ran} A$ is the range of $A$.

(iii) On $\bigcup_{a>0} \operatorname{Ran} E([a, \infty): \Gamma(\omega, L, E))$, it holds that

$$
\begin{aligned}
{\left[\left(\widehat{H_{L}^{\omega}}-E\right)^{1 / 2}, \Xi(\omega, L, E ; \iota, m)\right]_{+} } \\
=-\left(\widehat{H_{L}^{\omega}}-E\right)^{-1 / 2} \sum_{j=1}^{d}\left[\widetilde{\chi_{L}} \overline{e_{\iota, m}}(x) \cdot \nabla_{w} A_{j}\left(x, \overline{W_{\omega}}(x)\right),\right. \\
\left.\quad i \partial_{j}+\widetilde{\chi_{L}} A_{j}\left(x, \overline{W_{\omega}}(x)\right)\right]_{+}\left(\widehat{H_{L}^{\omega}}-E\right)^{-1 / 2} .
\end{aligned}
$$


(iv) For any $f \in C_{0}^{1}((0, \infty))$, we have

$$
\begin{aligned}
& \left\{\frac{\partial}{\partial \varepsilon} \operatorname{Tr}\left[f^{q}(\Gamma(\omega, L, E, \varepsilon ; \iota, m))\right]\right\}_{\varepsilon=0} \\
& =\operatorname{Tr}\left[\left(f^{q}\right)^{\prime}(\Gamma(\omega, L, E)) \Gamma(\omega, L, E ; \iota, m)\right],
\end{aligned}
$$

where

$$
\begin{aligned}
& \Gamma(\omega, L, E ; \iota, m) \\
& :=\left(\widehat{H_{L}^{\omega}}-E\right)^{-1 / 2} \widetilde{\chi_{L}} n e_{\iota, m}^{0}(x) W_{\omega}^{0}(x)^{n-1}\left(\widehat{H_{L}^{\omega}}-E\right)^{-1 / 2} \\
& \quad-\Gamma(\omega, L, E)\left(\widehat{H_{L}^{\omega}}-E\right)^{-1 / 2} \sum_{j=1}^{d}\left[\widetilde{\chi_{L}} \overline{e_{\iota, m}}(x) \cdot \nabla_{w} A_{j}\left(x, \overline{W_{\omega}}(x)\right),\right. \\
& \left.\quad i \partial_{j}+\widetilde{\chi_{L}} A_{j}\left(x, \overline{W_{\omega}}(x)\right)\right]_{+}\left(\widehat{H_{L}^{\omega}}-E\right)^{-1 / 2} .
\end{aligned}
$$

We postpone the proof of this lemma to the next subsection.

We use a vector field $\mathbb{A}$ on the space of sample paths defined by

$$
\mathbb{A} F\left(W_{\omega}(\cdot)\right)=\sum_{\iota=1}^{2 r} \sum_{m \in \mathbb{Z}^{d}} W_{\omega}^{\iota, m}\left(\frac{\partial}{\partial \varepsilon} F\left(W_{\omega}(\cdot, \varepsilon ; \iota, m)\right)\right)_{\varepsilon=0}
$$

in $L^{1}(\Omega)$, for any functional $F$ of the sample path $W_{\omega}(\cdot)$, when the limit exists. For this vector field, we have the following:

Lemma 2.4. For any $f \in C_{0}^{1}((0, \infty))$, we have

$$
\mathbb{A} \operatorname{Tr}\left[f^{q}(\Gamma(\omega, L, E))\right]=\operatorname{Tr}\left[\left(f^{q}\right)^{\prime}(\Gamma(\omega, L, E)) \Gamma^{(1)}(\omega, L, E)\right],
$$

where

$$
\begin{aligned}
& \Gamma^{(1)}(\omega, L, E) \\
& :=\Gamma(\omega, L, E)\left\{n-\left(\widehat{H_{L}^{\omega}}-E\right)^{-1 / 2} \sum_{j=1}^{d}\left[\widetilde{\chi_{L}} \overline{W_{\omega}}(x) \cdot \nabla_{w} A_{j}\left(x, \overline{W_{\omega}}(x)\right),\right.\right. \\
& \left.\left.\quad i \partial_{j}+\widetilde{\chi_{L}} A_{j}\left(x, \overline{W_{\omega}}(x)\right)\right]_{+}\left(\widehat{H_{L}^{\omega}}-E\right)^{-1 / 2}\right\} .
\end{aligned}
$$


Proof. By Lemma 2.3 (iv), we have

$$
\begin{aligned}
\mid \sum_{\iota=1}^{2 r} \sum_{|m|_{\infty} \leq M} W_{\omega}^{\iota, m}\left(\frac{\partial}{\partial \varepsilon} \operatorname{Tr}\left[f^{q}(\Gamma(\omega, L, E, \varepsilon ; \iota, m))\right]\right)_{\varepsilon=0} \\
-\operatorname{Tr}\left[\left(f^{q}\right)^{\prime}(\Gamma(\omega, L, E)) \Gamma^{(1)}(\omega, L, E)\right] \mid \\
\leq \mid \operatorname{Tr}\left[\left(f^{q}\right)^{\prime}(\Gamma(\omega, L, E))\left(\widehat{H_{L}^{\omega}}-E\right)^{-1 / 2} \widetilde{\chi_{L}} n W_{\omega}^{0}(x)^{n-1}\right. \\
\left.\quad \times R_{\omega}^{0}(x, M)\left(\widehat{H_{L}^{\omega}}-E\right)^{-1 / 2}\right] \mid \\
+\mid \operatorname{Tr}\left[\left(f^{q}\right)^{\prime}(\Gamma(\omega, L, E)) \Gamma(\omega, L, E)\left(\widehat{H_{L}^{\omega}}-E\right)^{-1 / 2}\right. \\
\quad \times \sum_{j=1}^{d}\left[\widetilde{\chi_{L}} \overline{R_{\omega}}(x, M) \cdot \nabla_{w} A_{j}\left(x, \overline{W_{\omega}}(x)\right),\right. \\
\left.\left.\quad i \partial_{j}+\widetilde{\chi_{L}} A_{j}\left(x, \overline{W_{\omega}}(x)\right)\right]_{+}\left(\widehat{H_{L}^{\omega}}-E\right)^{-1 / 2}\right] \mid,
\end{aligned}
$$

where

$$
R_{\omega}^{j}(x, M):=\sum_{\iota=1}^{2 r} \sum_{|m|_{\infty} \leq M} W_{\omega}^{\iota, m} e_{\iota, m}^{j}(x)-W_{\omega}^{j}(x)
$$

and $\overline{R_{\omega}}(x, M):={ }^{t}\left(R_{\omega}^{1}(x, M), \ldots, R_{\omega}^{d}(x, M)\right)$. By the Hölder inequality for the norms $\left|\|\cdot \mid\|_{p}\right.$ (cf. Theorem 2.8 in [33]), this is less than or equal to

$$
\begin{aligned}
& \|\|\left(f^{q}\right)^{\prime}(\Gamma(\omega, L, E))||_{q /(q-2)} \mid \|\left(\widehat{H_{L}^{\omega}}-E\right)^{-1 / 2} \widetilde{\chi_{L}} n W_{\omega}^{0}(x)^{n-1} \\
& \quad \times R_{\omega}^{0}(x, M)\left(\widehat{H_{L}^{\omega}}-E\right)^{-1 / 2} \|\left.\right|_{q / 2} \\
& +\|\|\left(f^{q}\right)^{\prime}(\Gamma(\omega, L, E)) \Gamma(\omega, L, E) \|\left.\right|_{q /(q-1)} \\
& \left.\times \| \mid \widehat{\mid\left(H_{L}^{\omega}\right.}-E\right)^{-1 / 2} \sum_{j=1}^{d}\left[\widetilde{\chi_{L}} \overline{R_{\omega}}(x, M) \cdot \nabla_{w} A_{j}\left(x, \overline{W_{\omega}}(x)\right),\right. \\
& \left.\quad i \partial_{j}+\widetilde{\chi_{L}} A_{j}\left(x, \overline{W_{\omega}}(x)\right)\right]_{+}\left(\widehat{H_{L}^{\omega}}-E\right)^{-1 / 2} \|\left.\right|_{q} .
\end{aligned}
$$

For any $p>1$, we have

$$
\begin{aligned}
\left\|\left(f^{q}\right)^{\prime}(\Gamma(\omega, L, E))\right\| \|_{p} & \leq c \operatorname{Tr}[E(\operatorname{supp} f: \Gamma(\omega, L, E))]^{1 / p} \\
& \leq c\||| \Gamma(\omega, L, E)\| \|_{q / 2}^{q /(2 p)}
\end{aligned}
$$

Similarly we have

$$
\|\|\left(f^{q}\right)^{\prime}(\Gamma(\omega, L, E)) \Gamma(\omega, L, E)\left|\left\|_{p} \leq c\left|\|\Gamma(\omega, L, E) \mid\|_{q / 2}^{q /(2 p)}\right.\right.\right.
$$


Thus, by Lemma 2.1, the quantity in (2.10) is dominated by

$$
\begin{aligned}
& \left\|\widetilde{\chi_{L}} W_{\omega}^{0}(x)^{n}\right\|_{q / 2}^{(q-2) / 2}\left\|\widetilde{\chi_{L}} n W_{\omega}^{0}(x)^{n-1} R_{\omega}^{0}(x, M)\right\|_{q / 2} \\
& +\left\|\widetilde{\chi_{L}} W_{\omega}^{0}(x)^{n}\right\|_{q / 2}^{(q-1) / 2} \sum_{j=1}^{d}\left\|\widetilde{\chi_{L}} \overline{R_{\omega}}(x, M) \cdot \nabla_{w} A_{j}\left(x, W_{\omega}^{j}(x)\right)\right\|_{q} .
\end{aligned}
$$

By the Hölder inequality on the probability space, we have

$$
\begin{aligned}
& E\left[\mid \sum_{\iota=1}^{2 r} \sum_{|m|_{\infty} \leq M} W_{\omega}^{\iota, m}\left(\frac{\partial}{\partial \varepsilon} \operatorname{Tr}\left[f^{q}(\Gamma(\omega, L, E, \varepsilon ; \iota, m))\right]\right)_{\varepsilon=0}\right. \\
& \left.\quad-\operatorname{Tr}\left[\left(f^{q}\right)^{\prime}(\Gamma(\omega, L, E)) \Gamma^{(1)}(\omega, L, E)\right] \mid\right] \\
& \leq c\left(E\left[\left\|\widetilde{\chi_{L}} W_{\omega}^{0}(x)^{n}\right\|_{q / 2}^{q / 2}\right]^{(q-2) / q} E\left[\left\|\widetilde{\chi_{L}} n W_{\omega}^{0}(x)^{n-1} R_{\omega}^{0}(x, M)\right\|_{q / 2}^{q / 2}\right]^{2 / q}\right. \\
& \left.\quad+E\left[\left\|\widetilde{\chi_{L}} W_{\omega}^{0}(x)^{n}\right\|_{q / 2}^{q / 2}\right]^{(q-1) / q} \sum_{j=1}^{d} E\left[\left\|\widetilde{\chi_{L}} \overline{R_{\omega}}(x, M) \cdot \nabla_{w} A_{j}\left(x, \overline{W_{\omega}}(x)\right)\right\|_{q}^{q}\right]^{1 / q}\right) .
\end{aligned}
$$

This converges to 0 as $M \rightarrow \infty$ since, for any $p>1, W_{\omega}^{0}(x) \in L^{p}\left(\Lambda_{L} \times \Omega\right)$ and $R_{\omega}(x, M)$ converges to 0 in $L^{p}\left(\Lambda_{L} \times \Omega\right)$ by Lemma 2.2 .

We now obtain

$$
\begin{aligned}
& E\left[\operatorname{Tr}\left[\left(-\rho_{\kappa}\right)^{\prime}(\Gamma(\omega, L, E)-1+t) \Gamma^{(1)}(\omega, L, E)\right]\right] \\
& =\sum_{\iota=1}^{2 r} \sum_{m \in \mathbb{Z}^{d}} I(L, E, t ; \iota, m)
\end{aligned}
$$

where

$$
\begin{aligned}
& I(L, E, t ; \iota, m) \\
& :=E\left[W_{\omega}^{\iota, m} \operatorname{Tr}\left[\left(-\rho_{\kappa}\right)^{\prime}(\Gamma(\omega, L, E)-1+t) \Gamma(\omega, L, E ; \iota, m)\right]\right] .
\end{aligned}
$$

Since

$$
\begin{aligned}
& \left(\varphi,\left(\widehat{H_{L}^{\omega}}-E\right)^{-1 / 2} \sum_{j=1}^{d}\left[\widetilde{\chi_{L}} W_{\omega}^{j}(x) \cdot \nabla_{w} A_{j}\left(x, \overline{W_{\omega}}(x)\right)\right.\right. \\
& \left.\left.i \partial_{j}+\widetilde{\chi_{L}} A_{j}\left(x, \overline{W_{\omega}}(x)\right)\right]_{+}\left(\widehat{H_{L}^{\omega}}-E\right)^{-1 / 2} \varphi\right) \\
& \leq 2\left\|w \cdot \nabla_{w} A\right\|_{\infty} / \sqrt{|E|}
\end{aligned}
$$


for any $\varphi \in L^{2}\left(\mathbb{R}^{d}\right)$ such that $\|\varphi\|_{2}=1$, we obtain

$$
\sum_{\iota=1}^{2 r} \sum_{m \in \mathbb{Z}^{d}} I(L, E, t ; \iota, m) \geq c(n, \kappa, E) E\left[\operatorname{Tr}\left[\left(-\rho_{\kappa}\right)^{\prime}(\Gamma(\omega, L, E)-1+t)\right]\right]
$$

for $-3 \kappa / 2 \leq t \leq 3 \kappa / 2$, where

$$
c(n, \kappa, E)=(1-2 \kappa)\left(n-2\left\|w \cdot \nabla_{w} A\right\|_{\infty} / \sqrt{|E|}\right)_{+} .
$$

If $E$ is less than $-\left(2\left\|w \cdot \nabla_{w} A\right\|_{\infty} / n\right)^{2}$ defined in (2.4), then $c(n, \kappa, E)$ does not vanish for small enough $\kappa$ and we have the following bound:

$$
\begin{aligned}
& P\left(\operatorname{dist}\left(E, \sigma\left(\widetilde{H_{L}^{\omega}}\right)\right) \leq \eta\right) \\
& \leq \frac{1}{c(n, \kappa, E)} \int_{-3 \kappa / 2}^{3 \kappa / 2} d t \sum_{\iota=1}^{2 r} \sum_{m \in \mathbb{Z}^{d}} I(L, E, t ; \iota, m) .
\end{aligned}
$$

We estimate the each term in the summation:

Lemma 2.5. $\quad$ There exists a finite constant $c$ independent of $L, E, m$ and $\kappa$ such that

$$
\sup _{-3 \kappa / 2 \leq t \leq 3 \kappa / 2}|I(L, E, t ; \iota, m)| \leq c L^{d} /|E|^{(q-d) / 2}
$$

for any $1 \leq \iota \leq 2 r, m \in \mathbb{Z}^{d}$ and $0<\kappa<1 / 8$.

Proof. Since $\Gamma(\omega, L, E)$ should be greater than $1-2 \kappa$ for $\rho_{\kappa}^{\prime}(\Gamma(\omega, L, E)-$ $1+t)$ to be nonzero, we have

$$
\begin{aligned}
I(L, E, t ; \iota, m)=E & {\left[W _ { \omega } ^ { \iota , m } \operatorname { T r } \left[\left(-\rho_{\kappa}\right)^{\prime}(\Gamma(\omega, L, E)-1+t)\right.\right.} \\
& \times \tau(\Gamma(\omega, L, E)+2 \kappa) \Gamma(\omega, L, E ; \iota, m)]],
\end{aligned}
$$

where $\tau(\mu)=v(\mu)^{q}$ and $v$ is a smooth function on $\mathbb{R}$ such that $0 \leq v \leq 1$, $0 \leq v^{\prime} \leq 3$ on $\mathbb{R}, v=0$ on $(-\infty, 1 / 2]$ and $v=1$ on $[1, \infty)$. This is rewritten as

$$
I(L, E, t ; \iota, m)=\sum_{j=1}^{2} I_{j}(L, E, t ; \iota, m),
$$

where

$$
\begin{aligned}
I_{1}(L, E, t ; \iota, m)= & E\left[W _ { \omega } ^ { \iota , m } \operatorname { T r } \left[\left\{-\rho_{\kappa}(\cdot-1+t) \tau(\cdot+2 \kappa)\right\}^{\prime}(\Gamma(\omega, L, E))\right.\right. \\
& \times \Gamma(\omega, L, E ; \iota, m)]]
\end{aligned}
$$


and

$$
\begin{aligned}
I_{2}(L, E, t ; \iota, m)= & E\left[W _ { \omega } ^ { \iota , m } \operatorname { T r } \left[\rho_{\kappa}(\Gamma(\omega, L, E)-1+t)\right.\right. \\
& \left.\left.\times \tau^{\prime}(\Gamma(\omega, L, E)+2 \kappa) \Gamma(\omega, L, E ; \iota, m)\right]\right] .
\end{aligned}
$$

By Lemma 2.3 (iv), we have

$$
\begin{aligned}
& I_{1}(L, E, t ; \iota, m) \\
& =E\left[\left(1-\left(W_{\omega}^{\iota, m}\right)^{2}\right) \operatorname{Tr}\left[\rho_{\kappa}(\Gamma(\omega, L, E)-1+t) \tau(\Gamma(\omega, L, E)+2 \kappa)\right]\right] .
\end{aligned}
$$

In fact, if we denote $\Gamma_{\iota, m, \lambda}(\omega, L, E)$ the operator obtained by replacing $W_{\omega}^{\iota, m}$ by $\lambda$ in $\Gamma(\omega, L, E)$, then $(2.14)$ is rewritten as

$$
\begin{aligned}
& E\left[\int _ { \mathbb { R } } \frac { d \lambda } { \sqrt { 2 \pi } } e ^ { - \lambda ^ { 2 } / 2 } \lambda \frac { \partial } { \partial \lambda } \operatorname { T r } \left[-\rho_{\kappa}\left(\Gamma_{\iota, m, \lambda}(\omega, L, E)-1+t\right)\right.\right. \\
& \left.\left.\quad \times \tau\left(\Gamma_{\iota, m, \lambda}(\omega, L, E)+2 \kappa\right)\right]\right] \\
& =E\left[\int _ { \mathbb { R } } \frac { d \lambda } { \sqrt { 2 \pi } } e ^ { - \lambda ^ { 2 } / 2 } ( 1 - \lambda ^ { 2 } ) \operatorname { T r } \left[\rho_{\kappa}\left(\Gamma_{\iota, m, \lambda}(\omega, L, E)-1+t\right)\right.\right. \\
& \left.\left.\quad \times \tau\left(\Gamma_{\iota, m, \lambda}(\omega, L, E)+2 \kappa\right)\right]\right]
\end{aligned}
$$

We here simply estimate as

$$
\begin{aligned}
& \left|\operatorname{Tr}\left[\rho_{\kappa}(\Gamma(\omega, L, E)-1+t) \tau(\Gamma(\omega, L, E)+2 \kappa)\right]\right| \\
& \leq \operatorname{Tr}[E((1 / 2-2 \kappa, \infty): \Gamma(\omega, L, E))] \\
& \leq(1 / 2-2 \kappa)^{-q / 2} \operatorname{Tr}\left[E((0, \infty): \Gamma(\omega, L, E)) \Gamma(\omega, L, E)^{q / 2}\right] \\
& \leq(1 / 2-2 \kappa)^{-q / 2} \mid\|\Gamma(\omega, L, E)\| \|_{q / 2}^{q / 2} \\
& \leq(1 / 2-2 \kappa)^{-q / 2} c_{d, q}\left\|\widetilde{\chi_{L}} W_{\omega}^{0}(x)^{n}\right\|_{q / 2}^{q / 2} /|E|^{(q-d) / 2}
\end{aligned}
$$

by Lemma 2.1 (ii). Then we obtain

$$
\begin{aligned}
\mid & I_{1}(L, E, t ; \iota, m) \mid \\
\leq & (1 / 2-2 \kappa)^{-q / 2} c_{d, q} \int_{\mathbb{R}^{d}} d x\left(\widetilde{\chi_{L}}(x)\right)^{q / 2} \\
& \times E\left[\left|1-\left(W_{\omega}^{\iota, m}\right)^{2}\right|\left|W_{\omega}^{0}(x)\right|^{n q / 2}\right] /|E|^{(q-d) / 2} \\
\leq & (1 / 2-2 \kappa)^{-q / 2} c_{d, q} L^{d} E\left[\left|1-\left(W_{\omega}^{\iota, m}\right)^{2}\right|^{2}\right]^{1 / 2} \\
& \times E\left[\left|W_{\omega}^{0}(0)\right|^{n q}\right]^{1 / 2} /|E|^{(q-d) / 2} .
\end{aligned}
$$


For $I_{2}(L, E, t ; \iota, m)$, we use Lemma 2.1 (ii) and (iii) to estimate parts of $\Gamma(\omega, L, E ; \iota, m)$ as

$$
\begin{aligned}
& \left\|\left(\widetilde{H_{L}^{\omega}}-E\right)^{-1 / 2} \widetilde{\chi_{L}} W_{\omega}^{0}(x)^{n}\left(\widetilde{H_{L}^{\omega}}-E\right)^{-1 / 2} \mid\right\|_{q / 2} \\
& \leq c_{d, q}\left\|\widetilde{\chi_{L}} W_{\omega}^{0}(x)^{n}\right\|_{q / 2} /|E|^{(q-d) / q}
\end{aligned}
$$

and

$$
\begin{gathered}
\|\|\left(\widetilde{H_{L}^{\omega}}-E\right)^{-1 / 2} \sum_{j=1}^{d}\left[\widetilde{\chi_{L}} \overline{e_{\iota, m}}(x) \cdot \nabla_{w} A_{j}\left(x, \overline{W_{\omega}}(x)\right),\right. \\
\left.i \partial_{j}+\widetilde{\chi_{L}} A_{j}\left(x, \overline{W_{\omega}}(x)\right)\right]_{+}\left(\widetilde{H_{L}^{\omega}}-E\right)^{-1 / 2}\|\|_{q} \\
\leq c_{d, q}\left\|\widetilde{\chi_{L}} \overline{e_{\iota, m}}(x) \cdot \nabla_{w} A_{j}\left(x, \overline{W_{\omega}}(x)\right)\right\|_{q} /|E|^{(q-d) /(2 q)} .
\end{gathered}
$$

On the other hand, as in (2.15), we have

$$
\begin{aligned}
& \left.\left\|\left|\rho_{\kappa}(\Gamma(\omega, L, E)-1+t) \tau^{\prime}(\Gamma(\omega, L, E)+2 \kappa)\right|\right\|\right|_{q /(q-2)} \\
& \leq 3(1 / 2-2 \kappa)^{-(q-2) / 2}\||\Gamma(\omega, L, E)|\|_{q / 2}^{(q-2) / 2}
\end{aligned}
$$

and

$$
\begin{aligned}
& \left\|\mid \rho_{\kappa}(\Gamma(\omega, L, E)-1+t) \tau^{\prime}(\Gamma(\omega, L, E)+2 \kappa) \Gamma(\omega, L, E)\right\| \|_{q /(q-1)} \\
& \leq 3(1 / 2-2 \kappa)^{-(q-3) / 2} \mid\|\Gamma(\omega, L, E)\|_{q / 2}^{(q-1) / 2} .
\end{aligned}
$$

Then, as in (2.16), we obtain

$$
\left|I_{2}(L, E, t ; \iota, m)\right| \leq(1 / 2-2 \kappa)^{-(q-2) / 2} c_{d, q} c_{R} L^{d} /|E|^{(q-d) / 2},
$$

where $c_{R}$ is a constant depending only on the covariance of $W_{\omega}^{0}(0)$.

This bound is simple and the growth as $L \rightarrow \infty$ is not big. However this does not decay as $|m|_{\infty} \rightarrow \infty$. To obtain the decay, we give another bound by using the spectral shift function as in [6], [7] and [20]:

Lemma 2.6. For any $\ell \in \mathbb{N}$ such that $\ell>q / 2$, there exists a finite constant $c$ independent of $L, m$ and $\kappa$ such that

$$
\begin{aligned}
& \sup _{-3 \kappa / 2 \leq t \leq 3 \kappa / 2}|I(L, E, t ; \iota, m)| \\
& \leq c|E|^{-(q-d) / 2}\left(|E|^{-q /(4 \ell)}+1\right)\left(\frac{L^{s-d / 2}}{\kappa|m|_{\infty}^{s}}\right)^{q /(2 \ell)} L^{d}
\end{aligned}
$$

for any $1 \leq \iota \leq 2 r, m \in \mathbb{Z}^{d}$ and $0<\kappa<1 / 2$. 
Proof. (2.14) is rewritten as

$$
\begin{aligned}
& I(L, E, t ; \iota, m) \\
& =E\left[( 1 - ( W _ { \omega } ^ { \iota , m } ) ^ { 2 } ) \operatorname { T r } \left[\rho_{\kappa}(\Gamma(\omega, L, E)-1+t)\right.\right. \\
& \left.\left.\quad-\rho_{\kappa}\left(\Gamma_{\iota, m}(\omega, L, E)-1+t\right)\right]\right],
\end{aligned}
$$

where $\Gamma_{\iota, m}(\omega, L, E)$ is the operator obtained by replacing $W_{\omega}^{\iota, m}$ by 0 in $\Gamma(\omega, L, E)$. In fact, $E\left[1-\left(W_{\omega}^{\iota, m}\right)^{2}\right]=0$ and $\Gamma_{\iota, m}(\omega, L, E)$ is statistically independent of $W_{\omega}^{\iota, m}$. By Lemma 2.7 below and Theorem 2.1 in [6], [7] and [20], we have the spectral shift function $\xi\left(\lambda: \Gamma(\omega, L, E)^{\ell}, \Gamma_{\iota, m}(\omega, L, E)^{\ell}\right)$ for the pair $\left(\Gamma(\omega, L, E)^{\ell}, \Gamma_{\iota, m}(\omega, L, E)^{\ell}\right)$ such that

$$
\begin{aligned}
& \left\|\xi\left(\cdot: \Gamma(\omega, L, E)^{\ell}, \Gamma_{\iota, m}(\omega, L, E)^{\ell}\right)\right\|_{2 \ell / q} \\
& \leq\|\| \Gamma(\omega, L, E)^{\ell}-\Gamma_{\iota, m}(\omega, L, E)^{\ell} \mid \|_{q /(2 \ell)}^{q /(2 \ell)} \\
& \leq\left\{c_{1}|E|^{-\ell(q-d) / q}\left(|E|^{-1 / 2}+1\right) Q(\omega, L, \iota, m)\right\}^{q /(2 \ell)} .
\end{aligned}
$$

As in [6], [7] and [20], we apply the Birman-Krein identity [2], [41] as follows:

$$
\begin{aligned}
& \operatorname{Tr}\left[\rho_{\kappa}(\Gamma(\omega, L, E)-1+t)-\rho_{\kappa}\left(\Gamma_{\iota, m}(\omega, L, E)-1+t\right)\right] \\
& =\int_{\mathbb{R}}\left\{\frac{\partial}{\partial \lambda} \rho_{\kappa}\left(\lambda^{1 / \ell}-1+t\right)\right\} \xi\left(\lambda: \Gamma(\omega, L, E)^{\ell}, \Gamma_{\iota, m}(\omega, L, E)^{\ell}\right) d \lambda .
\end{aligned}
$$

This is estimated as

$$
\begin{aligned}
& \left|\operatorname{Tr}\left[\rho_{\kappa}(\Gamma(\omega, L, E)-1+t)-\rho_{\kappa}\left(\Gamma_{\iota, m}(\omega, L, E)-1+t\right)\right]\right| \\
& \leq\left\{c_{1}|E|^{-\ell(q-d) / q}\left(|E|^{-1 / 2}+1\right) Q(\omega, L, \iota, m)\right\}^{q /(2 \ell)} \\
& \quad \times\left(\int_{\mathbb{R}}\left|\frac{\partial}{\partial \lambda} \rho_{\kappa}\left(\lambda^{1 / \ell}-1+t\right)\right|^{2 \ell /(2 \ell-q)} d \lambda\right)^{(2 \ell-q) /(2 \ell)}
\end{aligned}
$$

Since

$$
\begin{aligned}
& \left(\int_{\mathbb{R}}\left|\frac{\partial}{\partial \lambda} \rho_{\kappa}\left(\lambda^{1 / \ell}-1+t\right)\right|^{2 \ell /(2 \ell-q)} d \lambda\right)^{(2 \ell-q) /(2 \ell)} \\
& \leq c_{2}\left(\int_{\mathbb{R}}\left|\frac{\partial}{\partial \lambda} \rho_{\kappa}(\lambda-1+t)\right|^{2 \ell /(2 \ell-q)} d \lambda\right)^{(2 \ell-q) /(2 \ell)} \\
& \leq \frac{c_{3}}{\kappa^{q /(2 \ell)}}\left(\int_{\mathbb{R}}\left(-\frac{\partial}{\partial \lambda} \rho_{\kappa}\right)(\lambda-1+t) d \lambda\right)^{(2 \ell-q) /(2 \ell)} \\
& =c_{3} / \kappa^{q /(2 \ell)}
\end{aligned}
$$


and

$$
E\left[\left|1-\left(W_{\omega}^{\iota, m}\right)^{2}\right| Q(\omega, L, \iota, m)^{q /(2 \ell)}\right] \leq c_{4}\left(\frac{L^{s-d / 2}}{\kappa|m|_{\infty}^{s}}\right)^{q /(2 \ell)}
$$

we obtain $(2.17)$.

We next show the estimate of the difference of the operators used in the proof of the preceding lemma:

Lemma 2.7. For any $\ell \in \mathbb{N}$, the operator $\Gamma(\omega, L, E)^{\ell}-\Gamma_{\iota, m}(\omega, L, E)^{\ell}$ belongs to the super trace class $\mathcal{I}_{q /(2 \ell)}$ and satisfies

$$
\begin{aligned}
& \left\|\left|\Gamma(\omega, L, E)^{\ell}-\Gamma_{\iota, m}(\omega, L, E)^{\ell}\right|\right\|_{q /(2 \ell)} \\
& \leq c|E|^{-\ell(q-d) / q}\left(|E|^{-1 / 2}+1\right) Q(\omega, L, \iota, m),
\end{aligned}
$$

where $c$ is a finite constant,

$$
\begin{aligned}
& Q(\omega, L, \iota, m) \\
& :=\frac{L^{s-d / 2}}{|m|_{\infty}^{s}}\left|W_{\omega}^{\iota, m}\right| L^{d / q}\left(\left\|\widetilde{\chi_{L}} W_{\omega}^{0}(x)^{n}\right\|_{q}+\left\|\widetilde{\chi_{L}} W_{\omega, \iota, m}^{0}(x)^{n}\right\|_{q}+L^{d / q}\right) \\
& \quad \times\left(\left\|\widetilde{\chi_{L}} W_{\omega}^{0}(x)^{n}\right\|_{q / 2}+\left\|\widetilde{\chi_{L}} W_{\omega, \iota, m}^{0}(x)^{n}\right\|_{q / 2}\right. \\
& \left.\quad+\left\|\widetilde{\chi_{L}} \sum_{k=0}^{n-1} W_{\omega}^{0}(x)^{n-1+k} W_{\omega, \iota, m}^{0}(x)^{k}\right\|_{q / 2}+L^{2 d / q}\right) \\
& \quad \times\left(\left\|\widetilde{\chi_{L}} W_{\omega}^{0}(x)^{n}\right\|_{q / 2}+\left\|\widetilde{\chi_{L}} W_{\omega, \iota, m}^{0}(x)^{n}\right\|_{q / 2}+L^{2 d / q}\right)^{\ell-2}
\end{aligned}
$$

and $W_{\omega, \iota, m}^{0}(x)$ is the random field obtained by replacing $W_{\omega}^{\iota, m}$ by 0 in $W_{\omega}^{0}(x)$.

Proof. For simplicity, we treat only the case that $V(x, w)=0$. We divide the operator as

$$
\Gamma(\omega, L, E)^{\ell}-\Gamma_{\iota, m}(\omega, L, E)^{\ell}=\sum_{j=0}^{\ell} I_{j}+\sum_{j=1}^{\ell} J_{j}
$$


where

$$
\begin{aligned}
I_{0}:= & \left(\widehat{H_{L}^{\omega}}-E\right)^{-1 / 2} \widetilde{\chi_{L}} W_{\omega}^{0}(x)^{n}\left\{\left(\widehat{H_{L}^{\omega}}-E\right)^{-1} \widetilde{\chi_{L}} W_{\omega}^{0}(x)^{n}\right\}^{\ell-1} \\
& \times\left(\left(\widehat{H_{L}^{\omega}}-E\right)^{-1 / 2}-\left(\widehat{H_{L, \iota, m}^{\omega}}-E\right)^{-1 / 2}\right), \\
I_{j}:= & \left(\widehat{H_{L}^{\omega}}-E\right)^{-1 / 2}\left\{\widetilde{\chi_{L}} W_{\omega}^{0}(x)^{n}\left(\widehat{H_{L}^{\omega}}-E\right)^{-1}\right\}^{\ell-1-j} \widetilde{\chi_{L}} W_{\omega}^{0}(x)^{n} \\
& \times\left(\left(\widehat{H_{L}^{\omega}}-E\right)^{-1}-\left(\widehat{H_{L, \iota, m}^{\omega}}-E\right)^{-1}\right) \widetilde{\chi_{L}} W_{\omega, \iota, m}^{0}(x)^{n} \\
& \times\left\{\left(\widehat{H_{L, \iota, m}^{\omega}}-E\right)^{-1} \widetilde{\chi_{L}} W_{\omega, \iota, m}^{0}(x)^{n}\right\}^{j-1}\left(\widehat{H_{L, \iota, m}^{\omega}}-E\right)^{-1 / 2}
\end{aligned}
$$

for $1 \leq j \leq \ell-1$,

$$
\begin{aligned}
I_{\ell}:= & \left.\left(\widehat{H_{L}^{\omega}}-E\right)^{-1 / 2}-\left(\widehat{H_{L, \iota, m}^{\omega}}-E\right)^{-1 / 2}\right)\left\{\widetilde{\chi_{L}} W_{\omega, \iota, m}^{0}(x)^{n}\right. \\
& \left.\times\left(\widehat{H_{L, \iota, m}^{\omega}}-E\right)^{-1}\right\}^{\ell-1} \widetilde{\chi_{L}} W_{\omega, \iota, m}^{0}(x)^{n}\left(\widehat{H_{L, \iota, m}^{\omega}}-E\right)^{-1 / 2} \\
J_{j}:= & \left(\widehat{H_{L}^{\omega}}-E\right)^{-1 / 2}\left\{\widetilde{\chi_{L}} W_{\omega}^{0}(x)^{n}\left(\widehat{H_{L}^{\omega}}-E\right)^{-1}\right\}^{\ell-j} \\
& \times \widetilde{\chi_{L}}\left(W_{\omega}^{0}(x)^{n}-W_{\omega, \iota, m}^{0}(x)^{n}\right)\left\{\left(\widehat{H_{L, \iota, m}^{\omega}}-E\right)^{-1} \widetilde{\chi_{L}} W_{\omega, \iota, m}^{0}(x)^{n}\right\}^{j-1} \\
& \times\left(\widehat{H_{L, \iota, m}^{\omega}}-E\right)^{-1 / 2} \text { for } 1 \leq j \leq \ell
\end{aligned}
$$

and $\widehat{H_{L, \iota, m}^{\omega}}$ is the operator obtained by replacing $W_{\omega}^{\iota, m}$ by 0 in $\widehat{H_{L}^{\omega}}$. By the Hölder inequality for the norms $\|\mid \cdot\| \|_{p}$ (cf. Theorem 2.8 in [33]), we have

$$
\begin{aligned}
& \left\||| I_{0} \mid\right\|_{q /(2 \ell)} \\
& \leq||\left|\left(\widehat{H_{L}^{\omega}}-E\right)^{-1 / 2} \widetilde{\chi_{L}} W_{\omega}^{0}(x)^{n}\right|||_{q} \times \|\left.\left(\widehat{H_{L}^{\omega}}-E\right)^{-1} \widetilde{\chi_{L}} W_{\omega}^{0}(x)^{n}||\right|_{q / 2} ^{\ell-1} \\
& \quad \times\left.\left\|\left(\mid \widehat{H_{L}^{\omega}}-E\right)^{-1 / 2}-\left(\widehat{H_{L, \iota, m}^{\omega}}-E\right)^{-1 / 2}\right\|\right|_{q} .
\end{aligned}
$$

By Lemma 2.1 (i) and (iv), we have

$$
\left\|\left|\left(\widehat{H_{L}^{\omega}}-E\right)^{-1 / 2} \widetilde{\chi_{L}} W_{\omega}^{0}(x)^{n}\right|\right\|_{q} \leq c_{1}\left\|\widetilde{\chi_{L}} W_{\omega}^{0}(x)^{n}\right\|_{q} /|E|^{(q-d) /(2 q)}
$$

and

$$
\left\|\left.\left(\widehat{H_{L}^{\omega}}-E\right)^{-1} \widetilde{\chi_{L}} W_{\omega}^{0}(x)^{n}\left|\left\|_{q / 2} \leq c_{2}\right\| \widetilde{\chi_{L}} W_{\omega}^{0}(x)^{n} \|_{q / 2} /\right| E\right|^{(q-d) / q} .\right.
$$

By the expression

$$
\left(\widehat{H_{L}^{\omega}}-E\right)^{-1 / 2}=\frac{1}{\sqrt{\pi}} \int_{0}^{\infty} \frac{d t}{\sqrt{t}} e^{t E} \exp \left(-t \widehat{H_{L}^{\omega}}\right)
$$


((A.22) in [21]) and the Du Hamel formula (cf. [4]), we have

$$
\begin{aligned}
& \left(\widehat{H_{L}^{\omega}}-E\right)^{-1 / 2}-\left(\widehat{H_{L, \iota, m}^{\omega}}-E\right)^{-1 / 2} \\
& =\frac{1}{\sqrt{\pi}} \int_{0}^{\infty} d t \sqrt{t} e^{t E} \int_{0}^{1} d u \exp \left(-u t \widehat{H_{L}^{\omega}}\right) \\
& \quad \times \sum_{k=1}^{d}\left\{\left(i \partial_{k}+\widetilde{\chi_{L}} A_{k}\left(x, \overline{W_{\omega}}(x)\right)\right)\right. \\
& \quad \times \widetilde{\chi_{L}}\left(A_{k}\left(x, \overline{W_{\omega, \iota, m}}(x)\right)-A_{k}\left(x, \overline{W_{\omega}}(x)\right)\right) \\
& \quad+\widetilde{\chi_{L}}\left(A_{k}\left(x, \overline{W_{\omega, \iota, m}}(x)\right)-A_{k}\left(x, \overline{W_{\omega}}(x)\right)\right) \\
& \left.\quad \times\left(i \partial_{k}+\widetilde{\chi_{L}} A_{k}\left(x, \overline{W_{\omega, L, m}}(x)\right)\right)\right\} \exp \left(-(1-u) t \widehat{H_{L}^{\omega, L, m}}\right) .
\end{aligned}
$$

Since $\left\|\nabla_{w} A(x, w)\right\|_{\infty}<\infty$, this is estimated as

$$
\begin{aligned}
& \|\left.\left|\left(\widehat{H_{L}^{\omega}}-E\right)^{-1 / 2}-\left(\widehat{H_{L, \iota, m}^{\omega}}-E\right)^{-1 / 2}\right|\right|_{q} \\
& \leq c_{3}\left|W_{\omega}^{\iota, m}\right| \frac{L^{s-d / 2}}{|m|_{\infty}^{s}} \int_{0}^{\infty} d t \sqrt{t} e^{t E} \int_{0}^{1} d u \\
& \times \sum_{k=1}^{d}\left(||\left|\exp \left(-u t \widehat{H_{L}^{\omega}}\right)\left(i \partial_{k}+\widetilde{\chi_{L}} A_{k}\left(x, \overline{W_{\omega}}(x)\right)\right)\right| \|\right. \\
& \quad \times \| \widetilde{\chi_{L}} \exp \left(-(1-u) t \widehat{H_{L, \iota, m}^{\omega}}\right)||_{q} \\
&+\left\|\widetilde{\chi_{L}} \exp \left(-u t \widehat{H_{L}^{\omega}}\right)\left|\|_{q}\right|||\left(i \partial_{k}+\widetilde{\chi_{L}} A_{k}\left(x, \overline{W_{\omega}}(x)\right)\right)\right. \\
&\left.\times \exp \left(-(1-u) t \widehat{H_{L, \iota, m}^{\omega}}\right)|| \mid\right) .
\end{aligned}
$$

Since

$$
\left\|\mid \exp \left(-u t \widehat{H_{L}^{\omega}}\right)\left(i \partial_{k}+\widetilde{\chi_{L}} A_{k}\left(x, \overline{W_{\omega}}(x)\right)\right)\right\| \| \leq 1 / \sqrt{u t}
$$

and

$$
\begin{aligned}
& \left\|\widetilde{\chi_{L}} \exp \left(-u t \widehat{H_{L}^{\omega}}\right) \mid\right\|_{q} \\
& \leq\left\|\widetilde{\chi_{L}}\left(\widehat{H_{L}^{\omega}}-E\right)^{-1 / 2}\right\|\left\|_{q}\right\|\left|\left(\widehat{H_{L}^{\omega}}-E\right)^{1 / 2} \exp \left(-u t \widehat{H_{L}^{\omega}}\right)\right| \| \\
& \leq c_{4} L^{d / q} \sqrt{-E+1 /(u t)},
\end{aligned}
$$

we have

$$
\begin{aligned}
& ||\left|\left(\widehat{H_{L}^{\omega}}-E\right)^{-1 / 2}-\left(\widehat{H_{L, \iota, m}^{\omega}}-E\right)^{-1 / 2}\right| \|_{q} \\
& \leq c_{5}\left|W_{\omega}^{\iota, m}\right| \frac{L^{s-d / 2}}{|m|_{\infty}^{s}} L^{d / q} /|E|^{1-d /(2 q)}
\end{aligned}
$$


and

$$
\begin{aligned}
& \left\||| I_{0}|\||_{q /(2 \ell)}\right. \\
& \leq c_{6}\left|W_{\omega}^{\iota, m}\right| \frac{L^{s-d / 2}}{|m|_{\infty}^{s}} L^{d / q}\left\|\widetilde{\chi_{L}} W_{\omega}^{0}(x)^{n}\right\|_{q} \\
& \quad \times\left\|\widetilde{\chi_{L}} W_{\omega}^{0}(x)^{n}\right\|_{q / 2}^{\ell-1} /|E|^{\ell(q-d) / q+1 / 2} .
\end{aligned}
$$

$\left\||| I_{\ell} \mid\right\|_{q /(2 \ell)}$ is estimated similarly. By

$$
\begin{aligned}
& \left(\widehat{H_{L}^{\omega}}-E\right)^{-1}-\left(\widehat{H_{L, \iota, m}^{\omega}}-E\right)^{-1} \\
& =\left(\widehat{H_{L}^{\omega}}-E\right)^{-1} \sum_{k=1}^{d}\left\{\left(i \partial_{k}+\widetilde{\chi_{L}} A_{k}\left(x, \overline{W_{\omega}}(x)\right)\right)\right. \\
& \quad \times \widetilde{\chi_{L}}\left(A_{k}\left(x, \overline{W_{\omega, \iota, m}}(x)\right)-A_{k}\left(x, \overline{W_{\omega}}(x)\right)\right) \\
& \quad+\widetilde{\chi_{L}}\left(A_{k}\left(x, \overline{W_{\omega, \iota, m}}(x)\right)-A_{k}\left(x, \overline{W_{\omega}}(x)\right)\right) \\
& \left.\quad \times\left(i \partial_{k}+\widetilde{\chi_{L}} A_{k}\left(x, \overline{W_{\omega, \iota, m}}(x)\right)\right)\right\}\left(\widehat{H_{L, \iota, m}^{\omega}}-E\right)^{-1}
\end{aligned}
$$

and

$$
\left\|\left|\left(\widehat{H_{L}^{\omega}}-E\right)^{-1 / 2}\left(i \partial_{k}+\widetilde{\chi_{L}} A_{k}\left(x, \overline{W_{\omega}}(x)\right)\right)\right|\right\| \leq 1,
$$

we have

$$
\begin{aligned}
& \|\| I_{j}\|\|_{q /(2 \ell)} \\
& \leq c_{7}\left|W_{\omega}^{\iota, m}\right| \frac{L^{s-d / 2}}{|m|_{\infty}^{s}} L^{d / q}\left\{\left\|\widetilde{\chi_{L}} W_{\omega}^{0}(x)^{n}\right\|_{q / 2}^{\ell-1-j}\left\|\widetilde{\chi_{L}} W_{\omega}^{0}(x)^{n}\right\|_{q}\left\|\widetilde{\chi_{L}} W_{\omega, L, m}^{0}(x)^{n}\right\|_{q / 2}^{j}\right. \\
& \left.\quad+\left\|\widetilde{\chi_{L}} W_{\omega}^{0}(x)^{n}\right\|_{q / 2}^{\ell-j}\left\|\widetilde{\chi_{L}} W_{\omega, L, m}^{0}(x)^{n}\right\|_{q}\left\|\widetilde{\chi_{L}} W_{\omega, \iota, m}^{0}(x)^{n}\right\|_{q / 2}^{j-1}\right\} /|E|^{\ell(q-d) / q+1 / 2} .
\end{aligned}
$$

It is also easy to show

$$
\begin{aligned}
& \left\||| J_{j} \mid\right\|_{q /(2 \ell)} \\
& \leq c_{8}\left|X_{\omega}^{\iota, m}\right| \frac{L^{s-d / 2}}{|m|_{\infty}^{s}} L^{d / q}\left\|\widetilde{\chi_{L}} W_{\omega}^{0}(x)^{n}\right\|_{q}\left\|\widetilde{\chi_{L}} W_{\omega}^{0}(x)^{n}\right\|_{q / 2}^{\ell-1-j} \\
& \quad \times\left\|\sum_{k=1}^{n} \widetilde{\chi_{L}} W_{\omega}^{0}(x)^{n-k} W_{\omega, \iota, m}^{0}(x)^{k-1}\right\|_{q / 2}\left\|\widetilde{\chi_{L}} W_{\omega, L, m}^{0}(x)^{n}\right\|_{q / 2}^{j-1} /|E|^{\ell(q-d) / q}
\end{aligned}
$$

for $1 \leq j \leq \ell-1$ and

$$
\begin{aligned}
& \left\||| J_{\ell} \mid\right\|_{q /(2 \ell)} \\
& \leq c_{9}\left|W_{\omega}^{\iota, m}\right| \frac{L^{s-d / 2}}{|m|_{\infty}^{s}} L^{d / q}\left\|\sum_{k=1}^{n} \widetilde{\chi_{L}} W_{\omega}^{0}(x)^{n-k} W_{\omega, \iota, m}^{0}(x)^{k-1}\right\|_{q / 2} \\
& \quad \times\left\|\widetilde{\chi_{L}} W_{\omega, \iota, m}^{0}(x)^{n}\right\|_{q / 2}^{\ell-2}\left\|\widetilde{\chi_{L}} W_{\omega, \iota, m}^{0}(x)^{n}\right\|_{q} /|E|^{\ell(q-d) / q},
\end{aligned}
$$


where $a_{3}(\ell, q, d)$ is a number determined by $\ell, q$ and $d$.

To complete the proof of Theorem 2.1, we apply Lemma 2.5 to the terms with $|m|_{\infty} \leq L^{1-d /(2 s)} / \kappa^{1 / s}$ and Lemma 2.6 to other terms in (2.12). Then we obtain (2.4).

\section{§2.3. Proof of Lemma 2.3}

(i) As in (2.18), we have

$$
\begin{aligned}
& \left(\widehat{H_{L}^{\omega}}(\varepsilon ; \iota, m)-E\right)^{-1 / 2}-\left(\widehat{H_{L}^{\omega}}-E\right)^{-1 / 2} \\
& =\frac{1}{\sqrt{\pi}} \int_{0}^{\infty} d t \sqrt{t} e^{t E} \int_{0}^{1} d u \exp \left(-u t \widehat{H_{L}^{\omega}}(\varepsilon ; \iota, m)\right) \\
& \quad \times \sum_{j=1}^{d}\left\{\left(i \partial_{j}+\widetilde{\chi_{L}} A_{j}\left(x, \overline{W_{\omega}}(x, \varepsilon ; \iota, m)\right)\right)\right. \\
& \quad \times \widetilde{\chi_{L}}\left(A_{j}\left(x, \overline{W_{\omega}}(x)\right)-A_{j}\left(x, \overline{W_{\omega}}(x, \varepsilon ; \iota, m)\right)\right) \\
& \quad+\widetilde{\chi_{L}}\left(A_{j}\left(x, \overline{W_{\omega}}(x)\right)-A_{j}\left(x, \overline{W_{\omega}}(x, \varepsilon ; \iota, m)\right)\right) \\
& \left.\quad \times\left(i \partial_{j}+\widetilde{\chi_{L}} A_{j}\left(x, \overline{W_{\omega}}(x)\right)\right)\right\} \exp \left(-(1-u) t \widehat{H_{L}^{\omega}}\right) .
\end{aligned}
$$

Using

$$
\begin{aligned}
A_{j}\left(x, \overline{W_{\omega}}(x, \varepsilon ; \iota, m)\right)-A_{j}\left(x, \overline{W_{\omega}}(x)\right) \\
=\varepsilon \overline{e_{\iota, m}}(x) \cdot \int_{0}^{1} d v \nabla_{w} A_{j}\left(x, \overline{W_{\omega}}(x, \varepsilon v ; \iota, m)\right) \\
=\varepsilon \overline{e_{\iota, m}}(x) \cdot \nabla_{w} A_{j}\left(x, \overline{W_{\omega}}(x)\right) \\
\quad+\varepsilon \overline{e_{\iota, m}}(x) \cdot \int_{0}^{1} d v\left\{\nabla_{w} A_{j}\left(x, \overline{W_{\omega}}(x, \varepsilon v ; \iota, m)\right)-\nabla_{w} A_{j}\left(x, \overline{W_{\omega}}(x)\right)\right\},
\end{aligned}
$$

we expand as

$$
\begin{aligned}
& \left(\widehat{H_{L}^{\omega}}(\varepsilon ; \iota, m)-E\right)^{-1 / 2}-\left(\widehat{H_{L}^{\omega}}-E\right)^{-1 / 2} \\
& =\varepsilon \Xi(\omega, L, E ; \iota, m)+\sum_{j=1}^{3} \mathcal{R}_{j}(\varepsilon, \omega, L, E ; \iota, m),
\end{aligned}
$$


where

$$
\begin{aligned}
& \mathcal{R}_{1}(\varepsilon, \omega, L, E ; \iota, m):=\frac{-\varepsilon}{\sqrt{\pi}} \int_{0}^{\infty} d t \sqrt{t} e^{t E} \int_{0}^{1} d u \exp \left(-u t \widehat{H_{L}^{\omega}}(\varepsilon ; \iota, m)\right) \\
& \times \sum_{j=1}^{d}\left\{\left(i \partial_{j}+\widetilde{\chi_{L}} A_{j}\left(x, \overline{W_{\omega}}(x, \varepsilon ; \iota, m)\right)\right)\right. \\
& \quad \times \widetilde{\chi_{L}} \overline{e_{\iota, m}}(x) \cdot \int_{0}^{1} d v\left\{\nabla_{w} A_{j}\left(x, \overline{W_{\omega}}(x, \varepsilon v ; \iota, m)\right)-\nabla_{w} A_{j}\left(x, \overline{W_{\omega}}(x)\right)\right\} \\
& \quad+\widetilde{\chi_{L}} \overline{e_{\iota, m}}(x) \cdot \int_{0}^{1} d v\left\{\nabla_{w} A_{j}\left(x, \overline{W_{\omega}}(x, \varepsilon v ; \iota, m)\right)-\nabla_{w} A_{j}\left(x, \overline{W_{\omega}}(x)\right)\right\} \\
& \left.\quad \times\left(i \partial_{j}+\widetilde{\chi_{L}} A_{j}\left(x, \overline{W_{\omega}}(x)\right)\right)\right\} \exp \left(-(1-u) t \widehat{H_{L}^{\omega}}\right), \\
& \mathcal{R}_{2}(\varepsilon, \omega, L, E ; \iota, m):=\frac{-\varepsilon^{2}}{\sqrt{\pi}} \int_{0}^{\infty} d t \sqrt{t} e^{t E} \int_{0}^{1} d u \exp \left(-u t \widehat{H_{L}^{\omega}}(\varepsilon ; \iota, m)\right) \\
& \quad \times \sum_{j=1}^{d} \widetilde{\chi_{L}}{ }^{2} \overline{e_{\iota, m}}(x) \cdot \nabla_{w} A_{j}\left(x, \overline{W_{\omega}}(x)\right) \\
& \quad \times \int_{0}^{1} d v \overline{e_{\iota, m}}(x) \cdot \nabla_{w} A_{j}\left(x, \overline{W_{\omega}}(x, \varepsilon v ; \iota, m)\right) \exp \left(-(1-u) t \widehat{H_{L}^{\omega}}\right)
\end{aligned}
$$

and

$$
\begin{aligned}
& \mathcal{R}_{3}(\varepsilon, \omega, L, E ; \iota, m) \\
& :=\frac{-\varepsilon}{\sqrt{\pi}} \int_{0}^{\infty} d t \sqrt{t} e^{t E} \int_{0}^{1} d u\left(\exp \left(-u t \widehat{H_{L}^{\omega}}(\varepsilon ; \iota, m)\right)-\exp \left(-u t \widehat{H_{L}^{\omega}}\right)\right) \\
& \quad \times \sum_{j=1}^{d}\left[\widetilde{\chi_{L}} \overline{e_{\iota, m}}(x) \cdot \nabla_{w} A_{j}\left(x, \overline{W_{\omega}}(x)\right), i \partial_{j}+\widetilde{\chi_{L}} A_{j}\left(x, \overline{W_{\omega}}(x)\right)\right]_{+} \\
& \quad \times \exp \left(-(1-u) t \widehat{H_{L}^{\omega}}\right) .
\end{aligned}
$$

Since supp $\widetilde{\chi_{L}}$ is compact, for any $\varphi$ and $\psi \in L^{2}\left(\mathbb{R}^{d}\right)$, we have

$$
\begin{aligned}
& \quad\left|\left(\varphi, \mathcal{R}_{1}(\varepsilon, \omega, L, E ; \iota, m) \psi\right)\right| \\
& \leq o(\varepsilon) \int_{0}^{\infty} d t \sqrt{t} e^{t E} \int_{0}^{1} d u\left\{\|\left(i \nabla+\widetilde{\chi_{L}} A\left(x, \overline{W_{\omega}}(x, \varepsilon ; \iota, m)\right)\right)\right. \\
& \quad \times \exp \left(-u t \widehat{H_{L}^{\omega}}(\varepsilon ; \iota, m)\right) \varphi\left\|_{2}\right\| \psi \|_{2} \\
& \left.\quad+\|\varphi\|_{2}\left\|\left(i \nabla+\widetilde{\chi_{L}} A\left(x, \overline{W_{\omega}}(x)\right)\right) \exp \left(-(1-u) t \widehat{H_{L}^{\omega}}\right) \psi\right\|_{2}\right\} .
\end{aligned}
$$


Since

$$
\begin{aligned}
& \left\|\left(i \nabla+\widetilde{\chi_{L}} A\left(x, \overline{W_{\omega}}(x)\right)\right) \exp \left(-t \widehat{H_{L}^{\omega}}\right) \psi\right\|_{2} \\
& =\left(\exp \left(-t \widehat{H_{L}^{\omega}}\right) \psi, \widehat{H_{L}^{\omega}} \exp \left(-t \widehat{H_{L}^{\omega}}\right) \psi\right)^{1 / 2} \\
& \leq(2 t e)^{-1 / 2}\|\psi\|_{2},
\end{aligned}
$$

we have

$$
\left(\varphi, \mathcal{R}_{j}(\varepsilon, \omega, L, E ; \iota, m) \psi\right)=o(\varepsilon)
$$

for $j=1$. It is also easy to show (2.21) for $j=2$. For $\mathcal{R}_{3}(\varepsilon, \omega, L, E ; \iota, m)$, we use the Du Hamel formula again and apply similar estimates. Then we have (2.21) for $j=3$. By the same method, we can show that $\Xi(\omega, L, E ; \iota, m)$ is a bounded operator.

(ii) It is enough to show that $\widehat{H_{L}^{\omega}} \Xi(\omega, L, E ; \iota, m) E((a, \infty): \Gamma(\omega, L, E))$ is a bounded operator for any $a>0$. In this proof, we write $E^{j}:=\widetilde{\chi_{L}} \overline{e_{\iota, m}}(x)$. $\nabla_{w} A_{j}\left(x, \overline{W_{\omega}}(x)\right)$ and $A_{j}:=\widetilde{\chi_{L}} A_{j}\left(x, \overline{W_{\omega}}(x)\right)$, for simplicity. Since $\nabla_{w} A(x, w)$ is a $C^{2}$ function of $(x, w)$ and $\overline{W_{\omega}}(x)$ is a $C^{2}$ function of $x$ by Remark $2.3, E^{j}$ and $A^{j}$ are $C^{2}$ functions of $x$. Therefore, if we write $[A, B]_{-}=A B-B A$ for any operators $A$ and $B$, then we have

$$
\begin{aligned}
& {\left[\widehat{H_{L}^{\omega}}, \sum_{j=1}^{d}\left[E^{j}, i \partial_{j}+A_{j}\right]_{+}\right]_{-}} \\
& =\sum_{j, k=1}^{d}\left(i \partial_{j}+A_{j}\right) E_{(j, k)}\left(i \partial_{k}+A_{k}\right)+\sum_{k=1}^{d} E_{(k)}\left(i \partial_{k}+A_{k}\right)+E_{(0)}
\end{aligned}
$$

where $E_{(j, k)}, E_{(k)}$ and $E_{(0)}$ are continuous functions with compact support. By using this, we divide as follows:

$$
\widehat{H_{L}^{\omega}} \Xi(\omega, L, E ; \iota, m)=\sum_{j=1}^{7} H_{j},
$$


where

$$
\begin{aligned}
H_{1}:= & \frac{-2}{\sqrt{\pi}} \int_{0}^{\infty} d t \sqrt{t} e^{t E} \int_{0}^{1 / 2} d u \exp \left(-u t \widehat{H_{L}^{\omega}}\right) \sum_{j=1}^{d}\left(i \partial_{j}+A_{j}\right) E^{j} \widehat{H_{L}^{\omega}} \\
& \times \exp \left(-(1-u) t \widehat{H_{L}^{\omega}}\right), \\
H_{2}:= & \frac{1}{\sqrt{\pi}} \int_{0}^{\infty} d t \sqrt{t} e^{t E} \int_{0}^{1 / 2} d u \exp \left(-u t \widehat{H_{L}^{\omega}}\right) \sum_{j=1}^{d}\left(i \partial_{j} E^{j}\right) \widehat{H_{L}^{\omega}} \\
& \times \exp \left(-(1-u) t \widehat{H_{L}^{\omega}}\right), \\
H_{3}:= & \frac{-1}{\sqrt{\pi}} \int_{0}^{\infty} d t \sqrt{t} e^{t E} \int_{0}^{1 / 2} d u \exp \left(-u t \widehat{H_{L}^{\omega}}\right) \\
& \times \sum_{j, k=1}^{d}\left(i \partial_{j}+A_{j}\right) E_{(j, k)}\left(i \partial_{k}+A_{k}\right) \exp \left(-(1-u) t \widehat{H_{L}^{\omega}}\right), \\
H_{4}:= & \frac{-1}{\sqrt{\pi}} \int_{0}^{\infty} d t \sqrt{t} e^{t E} \int_{0}^{1 / 2} d u \exp \left(-u t \widehat{H_{L}^{\omega}}\right) \sum_{k=1}^{d} E_{(k)}\left(i \partial_{k}+A_{k}\right) \\
& \times \exp \left(-(1-u) t \widehat{H_{L}^{\omega}}\right), \\
& \frac{-1}{\sqrt{\pi}} \int_{0}^{\infty} d t \sqrt{t} e^{t E} \int_{0}^{1 / 2} d u \exp \left(-u t \widehat{H_{L}^{\omega}}\right) E_{(0)} \exp \left(-(1-u) t \widehat{H_{L}^{\omega}}\right), \\
H_{5}: & -2 \\
H_{6}:= & \sqrt{\pi} \int_{0}^{\infty} d t \sqrt{t} e^{t E} \int_{1 / 2}^{1} d u \widehat{H_{L}^{\omega}} \exp \left(-u t \widehat{H_{L}^{\omega}}\right) \sum_{j=1}^{d} E^{j}\left(i \partial_{j}+A_{j}\right) \\
& \times \exp \left(-(1-u) t \widehat{H_{L}^{\omega}}\right)
\end{aligned}
$$

and

$$
\begin{aligned}
H_{7}:= & \frac{-1}{\sqrt{\pi}} \int_{1 / 2}^{\infty} d t \sqrt{t} e^{t E} \int_{1 / 2}^{1} d u \widehat{H_{L}^{\omega}} \exp \left(-u t \widehat{H_{L}^{\omega}}\right) \\
& \times\left(\sum_{j=1}^{d} i \partial_{j} E^{j}\right) \exp \left(-(1-u) t \widehat{t H_{L}^{\omega}}\right) .
\end{aligned}
$$

As in the proof of (i), it is easy to show that $H_{2}, H_{3}, H_{4}, H_{5}$ and $H_{7}$ are bounded operators.

For $H_{1}$, we estimate as

$$
\begin{aligned}
& \left|\left(\varphi, \exp \left(-u t \widehat{H_{L}^{\omega}}\right) \sum_{j=1}^{d}\left(i \partial_{j}+A_{j}\right) E^{j} \widehat{H_{L}^{\omega}} \exp \left(-(1-u) t \widehat{H_{L}^{\omega}}\right) \psi\right)\right| \\
& \leq\left\|(i \nabla+A) \exp \left(-u t \widehat{H_{L}^{\omega}}\right) \varphi\right\|_{2}\left\|E^{\cdot}\right\|_{\infty}\left\|\widehat{H_{L}^{\omega}} \exp \left(-(1-u) t \widehat{H_{L}^{\omega}}\right) \psi\right\|_{2},
\end{aligned}
$$


and

$$
\left\|(i \nabla+A) \exp \left(-u t \widehat{H_{L}^{\omega}}\right) \varphi\right\|_{2} \leq c_{1}(u t)^{-1 / 2}\|\varphi\|_{2} .
$$

It is also easy to see that

$$
\left\|\widehat{H_{L}^{\omega}} \exp \left(-(1-u) \widehat{t H_{L}^{\omega}}\right) \psi\right\|_{2} \leq c_{2}((1-u) t)^{-1}\|\psi\|_{2} .
$$

However this bound is too big to integrate with respect to the variable $t$. We now take $\varphi$ from the range of $E((a, \infty): \Gamma(\omega, L, E))$. For this we have

$$
\begin{aligned}
& \left\|\left(\widehat{H_{L}^{\omega}}-E\right)^{1 / 2} \psi\right\|_{2}=\left\|\widetilde{\chi_{L}} X_{\omega}^{0}(x)^{n}\left(\widehat{H_{L}^{\omega}}-E\right)^{1 / 2} \Gamma(\omega, L, E)^{-1} \psi\right\|_{2} \\
& \leq c_{3}\left\|\Gamma(\omega, L, E)^{-1} \psi\right\|_{2} \leq c_{3}\|\psi\|_{2} / a .
\end{aligned}
$$

Then we have

$$
\left\|\left(\widehat{H_{L}^{\omega}}-E\right) \exp \left(-(1-u) \widehat{t H_{L}^{\omega}}\right) \psi\right\|_{2} \leq c_{4}\left(((1-u) t)^{-1 / 2}+1\right)\|\psi\|_{2} / a
$$

and

$$
\left\|\widehat{H_{L}^{\omega}} \exp \left(-(1-u) t \widehat{H_{L}^{\omega}}\right) \psi\right\|_{2} \leq c_{4}\left(((1-u) t)^{-1 / 2}+1\right)\|\psi\|_{2} / a+|E|\|\psi\|_{2} .
$$

Therefore $H_{1} E((a, \infty): \Gamma(\omega, L, E))$ is bounded. Similarly $H_{6} E((a, \infty)$ : $\Gamma(\omega, L, E))$ is bounded.

(iii) We take $\psi \in L^{2}\left(\mathbb{R}^{d}\right)$ and an element $\phi$ of the domain of $\widehat{H_{L}^{\omega}}$. We first show that

$$
\left(\psi,\left(\left(\widehat{H_{L}^{\omega}}-E\right)^{1 / 2}-\left(\widehat{H_{L}^{\omega}}(\varepsilon ; \iota, m)-E\right)^{1 / 2}\right) \phi\right)=O(\varepsilon)
$$

as $\varepsilon \rightarrow 0$. For this, we divide the left hand side as

$$
\begin{aligned}
& \left(\psi,\left(\left(\widehat{H_{L}^{\omega}}-E\right)^{-1 / 2}-\left(\widehat{H_{L}^{\omega}}(\varepsilon ; \iota, m)-E\right)^{-1 / 2}\right)\left(\widehat{H_{L}^{\omega}}-E\right) \phi\right) \\
& +\left(\psi,\left(\left(\widehat{H_{L}^{\omega}}(\varepsilon ; \iota, m)-E\right)^{-1 / 2}\left(\left(\widehat{H_{L}^{\omega}}-E\right)-\left(\widehat{H_{L}^{\omega}}(\varepsilon ; \iota, m)-E\right)\right) \phi\right) .\right.
\end{aligned}
$$

By (i), the first term is $O(\varepsilon)$. The second term is dominated by

$$
\varepsilon\|\psi\|_{2}\left(\left\|\left(i \nabla+\widetilde{\chi_{L}} A\left(x, \overline{W_{\omega}}(x)\right)\right) \phi\right\|_{2}+\|\phi\|_{2}\right) .
$$

Therefore we obtain (2.22).

We take $\varphi \in \bigcup_{a>0} \operatorname{Ran} E([a, \infty]: \Gamma(\omega, L, E))$. By (i), (ii) and (2.22), we have

$$
\begin{aligned}
& \left(\psi,\left[\left(\widehat{H_{L}^{\omega}}-E\right)^{1 / 2}, \Xi(\omega, L, E ; \iota, m)\right]_{+} \varphi\right) \\
& =\frac{1}{\varepsilon}\left(\psi,\left(\left(\widehat{H_{L}^{\omega}}(\varepsilon ; \iota, m)-E\right)^{1 / 2}\left(\left(\widehat{H_{L}^{\omega}}(\varepsilon ; \iota, m)-E\right)^{-1 / 2}-\left(\widehat{H_{L}^{\omega}}-E\right)^{-1 / 2}\right)\right.\right. \\
& \left.\left.\quad+\left(\left(\widehat{H_{L}^{\omega}}(\varepsilon ; \iota, m)-E\right)^{-1 / 2}-\left(\widehat{H_{L}^{\omega}}-E\right)^{-1 / 2}\right)\left(\widehat{H_{L}^{\omega}}-E\right)^{1 / 2}\right) \varphi\right)+O(\varepsilon) .
\end{aligned}
$$


Since $\left(\widehat{H_{L}^{\omega}}-E\right)^{1 / 2}\left(\widehat{H_{L}^{\omega}}-E\right)^{-1 / 2}=\left(\widehat{H_{L}^{\omega}}-E\right)^{-1 / 2}\left(\widehat{H_{L}^{\omega}}-E\right)^{1 / 2}=1$, the right hand side is equal to

$$
\begin{aligned}
\frac{-1}{\varepsilon}\left(\psi,\left(\left(\widehat{H_{L}^{\omega}}(\varepsilon ; \iota, m)-E\right)^{1 / 2}-\left(\widehat{H_{L}^{\omega}}-E\right)^{1 / 2}\right)\left(\widehat{H_{L}^{\omega}}(\varepsilon ; \iota, m)-E\right)^{-1 / 2}\right. \\
\left.+\left(\widehat{H_{L}^{\omega}}(\varepsilon ; \iota, m)-E\right)^{-1 / 2}\left(\left(\widehat{H_{L}^{\omega}}(\varepsilon ; \iota, m)-E\right)^{1 / 2}-\left(\widehat{H_{L}^{\omega}}-E\right)^{1 / 2}\right) \varphi\right) \\
+O(\varepsilon) \\
=\frac{-1}{\varepsilon}\left(\psi,\left(\widehat{H_{L}^{\omega}}(\varepsilon ; \iota, m)-E\right)^{-1 / 2}\left(\widehat{H_{L}^{\omega}}(\varepsilon ; \iota, m)-\widehat{H_{L}^{\omega}}\right)\right. \\
\left.\quad \times\left(\widehat{H_{L}^{\omega}}-E\right)^{-1 / 2} \varphi\right)+O(\varepsilon) .
\end{aligned}
$$

By (i) and (ii), we see that this is equal to

$$
\begin{gathered}
-\left(\psi,\left(\widehat{H_{L}^{\omega}}-E\right)^{-1} \sum_{j=1}^{d}\left[\widetilde{\chi_{L}} \overline{e_{\iota, m}}(x) \cdot \nabla_{w} A_{j}\left(x, \overline{W_{\omega}}(x)\right),\right.\right. \\
\left.\left.i \partial_{j}+\widetilde{\chi_{L}} A_{j}\left(x, \overline{W_{\omega}}(x)\right)\right]_{+}\left(\widehat{H_{L}^{\omega}}-E\right)^{-1 / 2} \varphi\right)+o(1) .
\end{gathered}
$$

(iv) As in [35], by the Fourier transform and the Du Hamel formula, we write as

$$
\begin{aligned}
& f(\Gamma(\omega, L, E, \varepsilon ; \iota, m))-f(\Gamma(\omega, L, E)) \\
& =\int_{\mathbb{R}} d \zeta \widehat{f}^{\prime}(\zeta) \int_{0}^{1} d u \exp (u 2 \pi i \zeta \Gamma(\omega, L, E, \varepsilon ; \iota, m)) \\
& \quad \times(\Gamma(\omega, L, E, \varepsilon ; \iota, m)-(\Gamma(\omega, L, E, \varepsilon)) \exp ((1-u) 2 \pi i \zeta \Gamma(\omega, L, E)) .
\end{aligned}
$$

By (i), we have

$$
\Gamma(\omega, L, E, \varepsilon ; \iota, m)-\Gamma(\omega, L, E, \varepsilon)=\varepsilon \Gamma^{\prime}(\omega, L, E ; \iota, m)+o(\varepsilon),
$$

where

$$
\begin{aligned}
\Gamma^{\prime}(\omega, & L, E ; \iota, m):=\left(\widehat{H_{L}^{\omega}}-E\right)^{-1 / 2} \widetilde{\chi_{L}} W_{\omega}^{0}(x)^{n} \Xi(\omega, L, E ; \iota, m) \\
& +\left(\widehat{H_{L}^{\omega}}-E\right)^{-1 / 2} \widetilde{\chi_{L}} n e_{\iota, m}^{0}(x) W_{\omega}^{0}(x)^{n-1}\left(\widehat{H_{L}^{\omega}}-E\right)^{-1 / 2} \\
& +\Xi(\omega, L, E ; \iota, m) \widetilde{\chi_{L}} W_{\omega}^{0}(x)^{n}\left(\widehat{H_{L}^{\omega}}-E\right)^{-1 / 2} .
\end{aligned}
$$

Then we obtain

$$
\begin{aligned}
& f(\Gamma(\omega, L, E, \varepsilon ; \iota, m))-f(\Gamma(\omega, L, E)) \\
& =\varepsilon \int_{\mathbb{R}} d \zeta \widehat{f}^{\prime}(\zeta) \int_{0}^{1} d u \exp (u 2 \pi i \zeta(\Gamma(\omega, L, E)) \\
& \quad \times \Gamma^{\prime}(\omega, L, E ; \iota, m) \exp ((1-u) 2 \pi i \zeta(\Gamma(\omega, L, E))+o(\varepsilon)
\end{aligned}
$$


We expand the trace as

$$
\operatorname{Tr}\left[f^{q}(\Gamma(\omega, L, E, \varepsilon ; \iota, m))\right]-\operatorname{Tr}\left[f^{q}(\Gamma(\omega, L, E))\right]=\sum_{k=1}^{q} I_{k},
$$

where

$$
\begin{aligned}
I_{k}:= & \sum_{1 \leq \ell_{1}<\cdots<\ell_{k} \leq q} \operatorname{Tr}[f(\Gamma(\omega, L, E)) \cdots \\
& \times\left(f(\Gamma(\omega, L, E, \varepsilon ; \iota, m)) \stackrel{\ell_{1}}{-} f(\Gamma(\omega, L, E))\right) \\
& \left.\times \cdots\left(f(\Gamma(\omega, L, E, \varepsilon ; \iota, m)) \stackrel{\ell_{k}}{-} f(\Gamma(\omega, L, E))\right) \cdots f(\Gamma(\omega, L, E))\right]
\end{aligned}
$$

Since supp $f \subset(0, \infty)$, the rank of $f(\Gamma(\omega, L, E))$ is finite. Thus, using (2.24), we have

$$
\begin{aligned}
I_{1} & =q \operatorname{Tr}\left[\{f(\Gamma(\omega, L, E ; \iota, m))-f(\Gamma(\omega, L, E))\} f^{q-1}(\Gamma(\omega, L, E))\right] \\
& =\varepsilon \operatorname{Tr}\left[\left(f^{q}\right)^{\prime}(\Gamma(\omega, L, E)) \Gamma^{\prime}(\omega, L, E ; \iota, m)\right]+o(\varepsilon) .
\end{aligned}
$$

By changing the order of the operators, we have

$$
\begin{aligned}
& \operatorname{Tr}\left[\left(f^{q}\right)^{\prime}(\Gamma(\omega, L, E)) \Gamma^{\prime}(\omega, L, E ; \iota, m)\right] \\
& =\operatorname{Tr}\left[\left(f^{q}\right)^{\prime}(\Gamma(\omega, L, E))\left(\widehat{H_{L}^{\omega}}-E\right)^{-1 / 2} \widetilde{\chi_{L}} n e_{\iota, m}^{0}(x) W_{\omega}^{0}(x)^{n-1}\left(\widehat{H_{L}^{\omega}}-E\right)^{-1 / 2}\right] \\
& \quad+\operatorname{Tr}\left[\left(f^{q}\right)^{\prime}(\Gamma(\omega, L, E)) \Gamma(\omega, L, E)\left[\left(\widehat{H_{L}^{\omega}}-E\right)^{1 / 2}, \Xi(\omega, L, E ; \iota, m)\right]_{+}\right] .
\end{aligned}
$$

By using also (iii), we obtain

$$
I_{1}=\varepsilon \operatorname{Tr}\left[\left(f^{q}\right)^{\prime}(\Gamma(\omega, L, E)) \Gamma(\omega, L, E ; \iota, m)\right]+o(\varepsilon) .
$$

Similarly we obtain

$$
I_{k}=O\left(\varepsilon^{k}\right)
$$

for $2 \leq k \leq q-1$. By (2.23), we have

$$
I_{q} \leq\left\|\widehat{f}^{\prime}\right\|_{1}^{q}\||\Gamma(\omega, L, E, \varepsilon ; \iota, m)-\Gamma(\omega, L, E)|\|_{q}^{q} .
$$

We divide the second factor as

$$
\begin{aligned}
& \|\| \Gamma(\omega, L, E, \varepsilon ; \iota, m)-\Gamma(\omega, L, E) \mid \|_{q} \\
& \leq\left\|\left|\left\|\left(\widehat{H_{L}^{\omega}}-E\right)^{-1 / 2} \widetilde{\chi_{L}} W_{\omega}^{0}(x)^{n} \mid\right\|_{q}\right.\right. \\
& \quad \times\left\|\left(\widehat{H_{L}^{\omega}}-E\right)^{-1 / 2}-\left(\widehat{H_{L}^{\omega}}(\varepsilon ; \iota, m)-E\right)^{-1 / 2} \mid\right\| \\
& \quad+\left\|\left|\left(\widehat{H_{L}^{\omega}}-E\right)^{-1 / 2}-\left(\widehat{H_{L}^{\omega}}(\varepsilon ; \iota, m)-E\right)^{-1 / 2}\right|\right\| \\
& \quad \times\left\|\widetilde{\chi_{L}} W_{\omega}^{0}(x, \varepsilon ; \iota, m)^{n}\left(\widehat{H_{L}^{\omega}}(\varepsilon ; \iota, m)-E\right)^{-1 / 2} \mid\right\|_{q} .
\end{aligned}
$$


By Lemma 2.1 (i), we have

$$
\begin{aligned}
& \left\|\left|\left(\widehat{H_{L}^{\omega}}-E\right)^{-1 / 2} \widetilde{\chi_{L}} W_{\omega}^{0}(x)^{n}\right|\right\|_{q}=O(1), \\
& \|\left.\left|\left(\widehat{H_{L}^{\omega}}-E\right)^{-1 / 2} \widetilde{\chi_{L}}\left(W_{\omega}^{0}(x)^{n}-W_{\omega}^{0}(x, \varepsilon ; \iota, m)^{n}\right)\right|\right|_{q}=O(\varepsilon)
\end{aligned}
$$

and

$$
\left\|\widetilde{\chi_{L}} W_{\omega}^{0}(x, \varepsilon ; \iota, m)^{n}\left(\widehat{H_{L}^{\omega}}(\varepsilon ; \iota, m)-E\right)^{-1 / 2}\right\|_{q}=O(1) .
$$

By (2.19) and (2.20), we have

$$
\left\|\left|\left(\widehat{H_{L}^{\omega}}-E\right)^{-1 / 2}-\left(\widehat{H_{L}^{\omega}}(\varepsilon ; \iota, m)-E\right)^{-1 / 2}\right|\right\|=O(\varepsilon) .
$$

Therefore we obtain

$$
\|\Gamma(\omega, L, E, \varepsilon ; \iota, m)-\Gamma(\omega, L, E)\|_{q}=O(\varepsilon)
$$

and

$$
I_{q}=O\left(\varepsilon^{q}\right)
$$

Lemma 2.3 is now proven.

\section{§3. Bootstrap Multiscale Analysis and Localization}

In this section we modify the theory in Germinet and Klein [14] so that the operator (2.5) can be treated. For this we weaken the assumptions on the Wegner estimate and the semiboundedness of the operator in [14]. In this section we treat a random operator

$$
H^{\omega}:=\sum_{j=1}^{d}\left(i \partial_{j}+\mathcal{A}_{j}^{\omega}(x)\right)^{2}+\mathcal{V}^{\omega}(x)
$$

with a general random field $\left(\mathcal{A}^{\omega}(x), \mathcal{V}^{\omega}(x)\right)$. We do not use the specific form of $\left(\mathcal{A}^{\omega}(x), \mathcal{V}^{\omega}(x)\right)$ in the last section. The application to the operator $(2.5)$ is discussed in Subsection 3.4 below. As in [14], we write $C=C(a, b, \ldots)$ if $C$ is a positive finite constant depending only on the parameters $a, b, \ldots$

\section{§3.1. Main theorems}

We first assume the following regularity and stationarity:

(R) For a.e. $\omega, \mathcal{V}^{\omega} \in L_{l o c}^{2}\left(\mathbb{R}^{d}\right)$ and $\mathcal{A}^{\omega}=\mathcal{A}^{(1), \omega}+\mathcal{A}^{(2), \omega}$, where $\mathcal{A}^{(1), \omega}$ and $\mathcal{A}^{(2), \omega}$ are satisfying the following:

(i) $\mathcal{A}^{(1), \omega} \in L_{l o c}^{4}\left(\mathbb{R}^{d} \rightarrow \mathbb{R}^{d}\right)$ and $\nabla \cdot \mathcal{A}^{(1), \omega} \in L_{l o c}^{2}\left(\mathbb{R}^{d}\right)$; 
(ii) $\mathcal{A}^{(2), \omega} \in C\left(\mathbb{R}^{d} \rightarrow \mathbb{R}^{d}\right), \nabla \cdot \mathcal{A}^{(2), \omega} \in L_{\text {loc }}^{2}\left(\mathbb{R}^{d}\right)$ and $d \mathcal{A}^{(2), \omega} \in C\left(\mathbb{R}^{d} \rightarrow\right.$ $\Lambda^{2}\left(\mathbb{R}^{d}\right)$ ), where $d$ is the exterior derivative of $\mathcal{A}^{(2), \omega}$ regarded as the 1 -form $\sum_{j=1}^{d} \mathcal{A}_{j}^{(2), \omega}(x) d x_{j}$ and $\Lambda^{2}\left(\mathbb{R}^{d}\right)$ is the 2 -fold exterior power of $\mathbb{R}^{d}$.

(S) The random field $\left(\mathcal{A}^{(1), \omega}(x), d \mathcal{A}^{(2), \omega}(x), \mathcal{V}^{\omega}(x)\right)$ is stationary with respect to the shift in the variable $x \in \mathbb{Z}^{d}$.

As in [14], we assume the independence at distance:

(IAD) There exists $\rho>0$ such that for any finite number of bounded subsets $B_{1}, B_{2}, \ldots, B_{m}$ of $\mathbb{R}^{d}$ with $\operatorname{dist}\left(B_{i}, B_{j}\right)>\rho$ for $i \neq j$, the random fields $\left\{\left(\mathcal{A}^{(1), \omega}(x), d \mathcal{A}^{(2), \omega}(x), \mathcal{V}^{\omega}(x)\right): x \in B_{i}\right\}, i=1,2, \ldots, m$, are independent.

Referring Lemma 3.5 below, we assume that a Fernique type estimate holds:

(F) There exist finite positive constants $\beta, \delta_{1}$ and $\delta_{2}$ such that

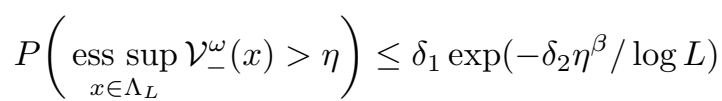

for any $L \geq 2$ and $\eta \geq 0$, where $\mathcal{V}_{-}^{\omega}(x):=\max \left\{-\mathcal{V}^{\omega}(x), 0\right\}$.

Under these assumptions the operator $H^{\omega}$ in (3.1) is essentially self-adjoint on $C_{0}^{\infty}\left(\mathbb{R}^{d}\right)$ by Lemma 3.1 below (cf. Leinfelder and Simader [29]).

Moreover, since the bound in Theorem 2.1 is not linear in the variable of energy, we assume that the Wegner estimate holds in a weaker form than that of [14]. For any $L>0$, let $H_{L}^{\omega}$ be the self-adjoint operator on $L^{2}\left(\Lambda_{L}\right)$ defined by the restriction of $H^{\omega}$ with the Dirichlet boundary condition. In the following we fix an open interval $\widetilde{I_{0}}$ and assume the following:

(W) There exist $1 \leq b<\infty, 0<h \leq 1$ and $0<Q_{\widetilde{I}_{0}}<\infty$ such that

$$
P\left(\operatorname{dist}\left(\sigma\left(H_{L}^{\omega}\right), E\right) \leq \eta\right) \leq Q_{\widetilde{I}_{0}} \eta^{h} L^{b d}
$$

for any $E \in \widetilde{I}_{0}, \eta>0$ and $L \in 2 \mathbb{N}$.

For any $x \in \mathbb{R}^{d}$ and $L>0$, let $\Lambda_{L}(x)$ be the open box of side $L$ centered at $x$. Let $H_{x, L}^{\omega}$ be the self-adjoint operator on $L^{2}\left(\Lambda_{L}(x)\right)$ defined by the restriction of $H^{\omega}$ with the Dirichlet boundary condition. Let $\chi_{x, L}$ and $\Gamma_{x, L}$ be the characteristic functions of $\Lambda_{L}(x)$ and $\overline{\Lambda_{L-1}(x)}-\Lambda_{L-3}(x)$, respectively. We use the following definitions in [14]:

Definition 3.1. Let $\theta>0, E \in \mathbb{R}, x \in \mathbb{Z}^{d}$ and $L \in 6 \mathbb{N}$. A box $\Lambda_{L}(x)$ is called $(\theta, E)$-suitable for $\omega$ if $E \notin \sigma\left(H_{x, L}^{\omega}\right)$ and

$$
\left\|\Gamma_{x, L}\left(H_{x, L}^{\omega}-E\right)^{-1} \chi_{x, L / 3}\right\| \mid \leq L^{-\theta} .
$$

Definition 3.2. $\quad$ Let $m>0, E \in \mathbb{R}, x \in \mathbb{Z}^{d}$ and $L \in 6 \mathbb{N}$. A box $\Lambda_{L}(x)$ is called $(m, E)$-regular for $\omega$ if $E \notin \sigma\left(H_{x, L}^{\omega}\right)$ and

$$
\left\||| \Gamma_{x, L}\left(H_{x, L}^{\omega}-E\right)^{-1} \chi_{x, L / 3}\right\| \mid \leq \exp (-m L / 2) .
$$


We fix a compact interval $I_{0}$ included in $\widetilde{I}_{0}$ and we set $M_{I_{0}}:=\max I_{0}$. Then the bootstrap multiscale analysis in [14] is modified as follows:

Theorem 3.1 (Bootstrap Multiscale Analysis, cf. Theorem 3.4 in [14]). For any $\theta>b d / h$, there exists $\overline{\mathcal{L}_{\theta}}=\overline{\mathcal{L}_{\theta}}\left(d, \rho, M_{I_{0}}, Q_{\widetilde{I_{0}}}, \beta, \delta_{1}, \delta_{2}, b, h, \theta\right) \in 6 \mathbb{N}$ satisfying the following: if there exist $E_{0} \in I_{0}$ and $\overline{\mathcal{L}_{\theta}} \leq \mathcal{L} \in 6 \mathbb{N}$ such that

$$
P\left(\Lambda_{\mathcal{L}} \text { is }\left(\theta, E_{0}\right)-\text { suitable }\right)>1-841^{-d},
$$

then there exists $\delta_{0}=\delta_{0}\left(d, \rho, M_{I_{0}}, Q_{\widetilde{I_{0}}}, \beta, \delta_{1}, \delta_{2}, \theta, \mathcal{L}\right)>0$ such that, for any $0<\zeta<1$ and $1<\alpha<\zeta^{-1}$, there are $L_{0}=L_{0}\left(d, \rho, M_{I_{0}}, Q_{\widetilde{I}_{0}}, \beta, \delta_{1}, \delta_{2}, \theta, \mathcal{L}, \zeta, \alpha\right)$ $\in 6 \mathbb{N}$ and $m_{\zeta}=m_{\zeta}\left(\zeta, L_{0}\right)>0$ satisfying

$$
P\left(R\left(m_{\zeta}, L_{k}, I_{0}\left(E_{0}, \delta_{0}\right), x, y\right)\right)>1-\exp \left(-L_{k}^{\zeta}\right)
$$

for any $k \in \mathbb{Z}_{+}$and $x, y \in \mathbb{Z}^{d}$ with $|x-y|_{\infty}>L_{k}+2 \rho$, where $L_{k+1}=\left[L_{k}^{\alpha}\right]_{6 \mathbb{N}}:=$ $\max \left\{N \in 6 \mathbb{N}: N \leq L_{k}^{\alpha}\right\}, I_{0}\left(E_{0}, \delta_{0}\right):=\left[E_{0}-\delta_{0}, E_{0}+\delta_{0}\right] \cap I_{0}$ and

$$
\begin{aligned}
& R(m, L, I, x, y) \\
& :=\left\{\omega: \text { for every } E \in I, \text { either } \Lambda_{L}(x) \text { or } \Lambda_{L}(y) \text { is }(m, E) \text {-regular }\right\} .
\end{aligned}
$$

As in [14], the following is obtained from Theorem 3.1 (cf. Theorem 3.8 in $[14])$ :

Theorem 3.2 (Decay of the kernel, cf. Theorem 3.8 in [14]). Let $\overline{\mathcal{L}_{\theta}}$ and $I_{0}\left(E_{0}, \delta_{0}\right)$ be the number and the interval given in Theorem 3.1. If there exists $E_{0} \in I_{0}$ such that (3.3) holds for some $\mathcal{L} \geq \overline{\mathcal{L}_{\theta}}$ and $\theta>b d / h$, then, for any $0<\zeta<1$, there exists a finite constant $C_{\zeta}=C_{\zeta}\left(\zeta, d, \rho, M_{I_{0}}, Q_{\widetilde{I}_{0}}, \beta, \delta_{1}, \delta_{2}\right.$, $b, h, \theta)$ such that

$$
\begin{aligned}
& E\left[\sup _{f \in \mathcal{B}_{1}(\mathbb{R})}|| \chi_{x} f\left(H^{\omega}\right) E\left(I_{0}\left(E_{0}, \delta_{0}\right): H^{\omega}\right) \chi_{y}||_{2}^{2}\right] \\
& \leq C_{\zeta} \exp \left(-|x-y|_{\infty}^{\zeta}\right)
\end{aligned}
$$

for any $x, y \in \mathbb{Z}^{d}$, where $|\cdot|_{\infty}$ is the maximal norm, $\chi_{x}$ is the operator of the multiplication with the characteristic function of the open box $\Lambda_{1}(x)$ with side 1 centered at $x$ and $\mathcal{B}_{1}(\mathbb{R})$ is the set of all real valued Borel functions $f$ on $\mathbb{R}$ with $\sup |f| \leq 1$. 
From this theorem, we obtain the strong dynamical localization in the Hilbert-Schmidt norm

$$
E\left[\sup _{t \in \mathbb{R}}\left|\left\|\left.|| X\right|^{n / 2} E\left(I_{0}\left(E_{0}, \delta_{0}\right): H^{\omega}\right) \exp \left(-i t H^{\omega}\right) \chi_{0}\right\|_{2}^{2}\right]<\infty\right.
$$

for any $n \geq 0$ (cf. Corollary 3.10 in [14]) and the strong dynamical localization in the operator norm

$$
E\left[\sup _{t \in \mathbb{R}}\left|\left\|\left.|| X\right|^{n / 2} E\left(I_{0}\left(E_{0}, \delta_{0}\right): H^{\omega}\right) \exp \left(-i t H^{\omega}\right) \chi_{0}\right\|\right|\right]<\infty
$$

for any $n \geq 0$ (cf. (1.8) in [14]), where $|X|^{n / 2}$ is the operators of multiplication with $|x|^{n / 2}$. The equivalence of these two notions shown in [16] holds also in our case as we discuss in Subsection 4.1 below. From these, we obtain the weak dynamical localization,

$$
\sup _{t \in \mathbb{R}}\left\|\left.|| X\right|^{n / 2} E\left(I_{0}\left(E_{0}, \delta_{0}\right): H^{\omega}\right) \exp \left(-i t H^{\omega}\right) \chi_{0} \mid\right\|<\infty \text { for a.e. } \omega
$$

and the absence of the continuous spectrum

$$
\sigma_{c}\left(H^{\omega}\right) \cap I_{0}\left(E_{0}, \delta_{0}\right)=\emptyset \text { for a.e. } \omega
$$

as is explained in [14] (cf. [37]).

On the other hand, we obtain the following from Theorem 3.1 as in [14]:

Theorem 3.3. $\quad$ (Semi Uniformly Localized Eigenfunction, cf. Theorem 3.11 in [14]). Let $\overline{\mathcal{L}_{\theta}}$ and $I_{0}\left(E_{0}, \delta_{0}\right)$ be the number and the interval given in Theorem 3.1. If there exists $E_{0} \in I_{0}$ such that (3.3) holds for some $\mathcal{L} \geq \overline{\mathcal{L}_{\theta}}$ and $\theta>b d / h$, then, for any $\varepsilon>0$, there exists $m_{\varepsilon}>0$ satisfying the following for a.e. $\omega: \sigma_{c}\left(H^{\omega}\right) \cap I_{0}\left(E_{0}, \delta_{0}\right)=\emptyset$ and, if $\left\{\phi_{j, \omega}\right\}_{j \in \mathbb{N}}$ is the normalized eigenfunctions of $H^{\omega}$ with energy $E_{j, \omega}$ in $I_{0}\left(E_{0}, \delta_{0}\right)$, then, for any $\nu>d / 4$, there exist $C_{\varepsilon, \omega}, \widetilde{C_{\omega}} \in(0, \infty)$ and $\left\{x_{j, \omega}\right\}_{j \in \mathbb{N}} \subset \mathbb{Z}^{d}$ such that

$$
\left\|\chi_{x} \phi_{j, \omega}\right\|_{2} \leq C_{\varepsilon, \omega} \exp \left(m_{\varepsilon}\left(\log \left|x_{j, \omega}\right|_{\infty}\right)_{+}^{1+\varepsilon}-m_{\varepsilon}\left|x-x_{j, \omega}\right|_{\infty}\right)
$$

and

$$
\left|x_{j, \omega}\right|_{\infty} \geq \widetilde{C_{\omega}} j^{1 /(4 \nu)}
$$

for any $j \in \mathbb{N}$ and $x \in \mathbb{Z}^{d}$. 
Moreover we assume that the coefficients belongs to the local Kato class $\mathcal{K}_{\text {loc }}\left(\mathbb{R}^{d}\right)$ defined as follows: a real function $f$ on $\mathbb{R}^{d}$ is said to be in the Kato class $\mathcal{K}\left(\mathbb{R}^{d}\right)$ if

$$
\limsup _{\alpha \downarrow 0} \int_{x \in \mathbb{R}} g_{|x-y| \leq \alpha}(x-y)|f(y)| d y=0
$$

and is said to be in the local Kato class $\mathcal{K}_{\text {loc }}\left(\mathbb{R}^{d}\right)$ if $\chi_{K} f \in \mathcal{K}_{\text {loc }}\left(\mathbb{R}^{d}\right)$ for all compact $K \subset \mathbb{R}^{d}$, where

$$
g_{d}(x):=\left\{\begin{array}{l}
-\log |x| \text { if } d=2 \\
|x|^{2-d} \text { if } d \geq 3 .
\end{array}\right.
$$

Then we obtain the following as in Lemma 2.5 in [22]:

Proposition 3.1. We assume

$$
\sum_{j=1}^{d}\left(\mathcal{A}_{j}^{(1), \omega}\right)^{2}, \nabla \cdot \mathcal{A}_{j}^{\omega}, \quad\left|\mathcal{V}^{\omega}\right| \in \mathcal{K}_{l o c}\left(\mathbb{R}^{d}\right) .
$$

Then, in the situation of Theorem 3.3 , the eigenfunction $\phi_{j, \omega}$ is a continuous function satisfying

$$
\left|\phi_{j, \omega}(x)\right| \leq C_{d, \varepsilon, \omega} \exp \left(m_{\varepsilon}^{\prime}\left(\log \left|x_{j, \omega}\right|_{\infty}\right)_{+}^{1+\varepsilon}-m_{\varepsilon}^{\prime}\left|x-x_{j, \omega}\right|_{\infty}\right),
$$

where $C_{d, \varepsilon, \omega}$ and $m_{\varepsilon}^{\prime}$ are finite constants independent of $j$.

\section{§3.2. Verification of Assumptions in [14]}

The theory in Germinet and Klein [14] is discussed under several assumptions in an abstract setting. We have already introduced some of these conditions. In this subsection we show that the other assumptions hold in their forms or in weakened forms.

We first prepare the following proposition, which we often use in the following:

Lemma 3.1. Under the assumption $(\mathrm{F})$, we have the following:

(i) For any $1 \leq p<\infty$, there exists a finite constant $c=c\left(\delta_{1}, \delta_{2}, \beta, p\right)$ such that

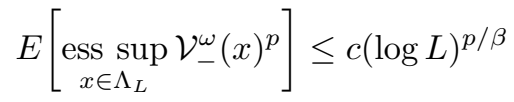

for any $L \geq 2$. 
(ii) For almost all $\omega$, there exists a finite constant $c_{\omega}$ such that

$$
\mathcal{V}_{-}^{\omega}(x) \leq c_{\omega}\left\{\log \left(|x|_{\infty}+2\right)\right\}^{2 / \beta}
$$

for almost every $x \in \mathbb{R}^{d}$.

Proof. (i) By Fubini's theorem, we have

$$
\begin{aligned}
E\left[\underset{x \in \Lambda_{L}}{\operatorname{ess} \sup _{-}} \mathcal{V}_{-}^{\omega}(x)^{p}\right] & =\int_{0}^{\infty} \lambda d_{\lambda} P\left(\underset{x \in \Lambda_{L}}{\operatorname{ess} \sup _{-}} \mathcal{V}_{-}^{\omega}(x)^{p} \leq \lambda\right) \\
& =\int_{0}^{\infty} P\left(\underset{x \in \Lambda_{L}}{\operatorname{ess} \sup _{-}} \mathcal{V}_{-}^{\omega}(x)^{p}>\eta\right) d \eta
\end{aligned}
$$

By applying (3.2) to the right hand side we obtain the result.

(ii) By taking $\delta_{3}>1 / \sqrt{\delta_{2}}$, we have

$$
\sum_{L \in \mathbb{N}} P\left(\operatorname{ess}_{x \in \Lambda_{L}} \operatorname{Vup}_{-}^{\omega}(x)>\left(\delta_{3} \log L\right)^{2 / \beta}\right)<\infty .
$$

By the Borel-Cantelli lemma, we see that, for almost all $\omega$, there exists $L_{\omega} \in \mathbb{N}$ such that

$$
\underset{x \in \Lambda_{L}}{\operatorname{ess} \sup _{-}} \mathcal{V}_{-}^{\omega}(x) \leq\left(\delta_{3} \log L\right)^{2 / \beta}
$$

for any $L \geq L_{\omega}$ Then for any $L \geq L_{\omega}$ and almost every $x \in \Lambda_{L}-\Lambda_{L-1}$, we have

$$
\mathcal{V}_{-}^{\omega}(x) \leq\left(\delta_{3} \log L\right)^{2 / \beta} \leq\left\{\delta_{3} \log \left(2|x|_{\infty}+1\right)\right\}^{2 / \beta} .
$$

Therefore we obtain the result.

We write $\Lambda_{L}(x) \sqsubset \Lambda_{L^{\prime}}\left(x^{\prime}\right)$ if $\Lambda_{L}(x) \subset \Lambda_{L^{\prime}-3}\left(x^{\prime}\right)$. Then the assumption (SLI) in [14] relating the Simon-Lieb inequality is verified in the following weakened form:

Proposition 3.2. There exists a finite constant $c=c\left(d, M_{I_{0}}\right)$ such that, for any $L, \ell^{\prime}, \ell^{\prime \prime} \geq 2, x, y, y^{\prime} \in \mathbb{R}^{d}$ with $\Lambda_{\ell^{\prime \prime}}(y) \sqsubset \Lambda_{\ell^{\prime}}\left(y^{\prime}\right) \sqsubset \Lambda_{L}(x)$ and any $E \in I_{0}-\sigma\left(H_{x, L}\right)-\sigma\left(H_{y^{\prime}, \ell^{\prime}}\right)$,

$$
\begin{aligned}
& \left\|\mid \Gamma_{x, L}\left(H_{x, L}-E\right)^{-1} \chi_{y, \ell^{\prime \prime}}\right\| \\
& \leq\left(\left(\underset{\Gamma_{y^{\prime}, \ell^{\prime}}}{\operatorname{ess} \sup } \mathcal{V}_{-}^{\omega}\right)^{1 / 2}+c\right)\left\||| \Gamma_{y^{\prime}, \ell^{\prime}}\left(H_{y^{\prime}, \ell^{\prime}}-E\right)^{-1} \chi_{y, \ell^{\prime \prime}}\right\| \| \\
& \quad \times\left\|\mid \Gamma_{x, L}\left(H_{x, L}-E\right)^{-1} \Gamma_{y^{\prime}, \ell^{\prime}}\right\| .
\end{aligned}
$$


The assumption (SLI) in [14] requires that the first factor in the right hand side of (3.7) is bounded in $\omega$. Instead of the boundedness, we use the assumption $(\mathrm{F})$.

To prove this proposition, we modify Lemma 2.5.3 in [37] as follows:

Lemma 3.2. There exists a finite constant $c=c(d)$ satisfying the following: let $\widetilde{B}, B$ be bounded open regions in $\mathbb{R}^{d}$ such that $\widetilde{B} \subset B$ and $\delta:=$ $\operatorname{dist}(\partial \widetilde{B}, \partial B)>0$. Let $g \in L^{2}(B)$ and $u \in L^{2}(B)$ such that $\left(i \nabla+\mathcal{A}^{\omega}\right) u \in L^{2}(B)$ and $\left(\left(i \nabla+\mathcal{A}^{\omega}\right) u,\left(i \nabla+\mathcal{A}^{\omega}\right) \varphi\right)+\left(u, \mathcal{V}^{\omega} \varphi\right)=(g, \varphi)$ for any $\varphi \in C_{0}^{\infty}(B)$. Then it holds that

$$
\left\|\left(i \nabla+\mathcal{A}^{\omega}\right) u\right\|_{L^{2}(\widetilde{B})} \leq\left(\left(\underset{B}{\operatorname{ess} \sup \mathcal{V}_{-}^{\omega}}\right)^{1 / 2}+\frac{c}{\delta}\right)\|u\|_{L^{2}(B)}+\|g\|_{L^{2}(B)} .
$$

Then Lemma 2.5.2 in [37] is modified as follows:

Lemma 3.3. There exists a finite constant $c=c\left(d, M_{I_{0}}\right)$ satisfying the following: let $\Lambda_{L^{\prime}}\left(x^{\prime}\right) \subset \Lambda_{L}(x)$ and $A^{\prime} \subset \Lambda_{L^{\prime}}\left(x^{\prime}\right), A \subset \Lambda_{L}(x)$. Let $\phi \in C_{0}^{1}\left(\Lambda_{L}(x)\right)$ and $B$ be an open neighborhood of $\operatorname{supp} \nabla \phi$ such that $\delta_{1}:=$ $\operatorname{dist}(\partial B, \operatorname{supp} \nabla \phi)>0, B \cap A=\emptyset$ and $B \subset \Lambda_{L}(x)$. Then it holds that

$$
\begin{aligned}
& \left\|\left|\chi_{A^{\prime}}\left(\phi\left(H_{x, L}-E\right)^{-1}-\left(H_{x^{\prime}, L^{\prime}}-E\right)^{-1} \phi\right) \chi_{A} \|\right|\right. \\
& \leq\left(\left(\underset{B}{\operatorname{ess} \sup } \mathcal{V}_{-}^{\omega}\right)^{1 / 2}+\frac{c}{\delta_{1}}\right)\left\|\chi_{A^{\prime}}\left(H_{x^{\prime}, L^{\prime}}-E\right)^{-1} \chi_{B}\right\| \mid \\
& \times\left\|\mid \chi_{B}\left(H_{x, L}-E\right)^{-1} \chi_{A}\right\| \|
\end{aligned}
$$

for any $E \in I_{0}-\sigma\left(H_{x, L}\right)-\sigma\left(H_{x^{\prime}, L^{\prime}}\right)$.

Proof of Proposition 3.2. We have only to take $\Lambda_{L^{\prime}}\left(x^{\prime}\right)=\Lambda_{\ell^{\prime}}\left(y^{\prime}\right), A^{\prime}=$ $\Lambda_{\ell^{\prime \prime}}(y), A=\overline{\Lambda_{L-1}(x)}-\Lambda_{L-3}(x), B=\Lambda_{\ell^{\prime}-1}\left(y^{\prime}\right)-\overline{\Lambda_{\ell^{\prime}-3}\left(y^{\prime}\right)}$ and $\phi$ so that $\phi=1$ on $\Lambda_{\ell^{\prime}-5 / 2}\left(y^{\prime}\right)$ and $\phi=0$ on $\Lambda_{\ell^{\prime}-3 / 2}\left(y^{\prime}\right)^{c}$ in Lemma 3.3 .

Let $T$ be the operator of multiplication with the function $\left(1+|x|^{2}\right)^{\nu}$, where $\nu$ is a fixed number greater than $d / 4$. Let $\mathcal{H}_{ \pm}$be the weighted spaces defined by

$$
\mathcal{H}_{ \pm}:=L^{2}\left(\mathbb{R}^{d},\left(1+|x|^{2}\right)^{ \pm 2 \nu} d x\right) .
$$

The set $\mathcal{D}_{+}^{\omega}:=\left\{\phi \in \mathcal{D}\left(H^{\omega}\right) \cap \mathcal{H}_{+}, H^{\omega} \phi \in \mathcal{H}_{+}\right\}$is dense in $\mathcal{H}_{+}$and an operator core for $H^{\omega}$, for a.e. $\omega$, where $\mathcal{D}\left(H^{\omega}\right)$ is the domain of $H^{\omega}$.

$\psi \in \mathcal{H}_{-}$is called a generalized eigenfunction of $H^{\omega}$ with generalized eigenvalue $E$ if

$$
\int \overline{H^{\omega} \varphi} \psi d x=E \int \bar{\varphi} \psi d x
$$


for any $\varphi \in \mathcal{D}_{+}^{\omega}$. Then the assumption (EDI) in [14] on an eigenfunction decay inequality is verified in the following weakened form:

Proposition 3.3. There exists a finite constant $c=c\left(d, M_{I_{0}}\right)$ satisfying the following: for any generalized eigenfunction $\psi$ of $H^{\omega}$ with generalized eigenvalue $E \in I_{0}, x \in \mathbb{R}^{d}$ and $L \geq 4$ such that $E \notin \sigma\left(H_{x, L}^{\omega}\right)$, it holds that

$$
\left\|\chi_{x} \psi\right\|_{2} \leq c\left(\left(\underset{\Lambda_{L}(x)}{\operatorname{ess} \sup _{-} \mathcal{V}_{-}^{\omega}}\right)^{1 / 2}+1\right)\left\|\mid \Gamma_{x, L}\left(H_{x, L}^{\omega}-E\right)^{-1} \chi_{x}\right\|\|\times\| \Gamma_{x, L} \psi \|_{2} .
$$

As in the assumption (SLI), the assumption (EDI) in [14] requires that the second factor in the right hand side of (3.8) is bounded in $\omega$. This proposition is proven by Lemma 3.2.

The assumption (NE) in [14] on the average number of eigenvalues is verified by the following:

Proposition 3.4. For any $a \in \mathbb{R}$, there exists a finite constant $C_{a}=$ $C_{a}\left(a, d, \beta, \delta_{1}, \delta_{2}\right)$ such that

$$
E\left[\sharp\left\{(-\infty, a) \cap \sigma\left(H_{L}^{\omega}\right)\right\}\right] \leq C_{a} L^{d}
$$

for any $L \geq 1$.

Proof. Let $H_{L}^{\omega, a}$ be the operator obtained by replacing $\mathcal{V}^{\omega}$ by $-\left(\mathcal{V}^{\omega}-a-\right.$ 1) _ in the definition of $H_{L}^{\omega}$. Then, since $H_{L}^{\omega}-a-1 \geq H_{L}^{\omega, a}$, we have

$$
\sharp\left\{(-\infty, a) \cap \sigma\left(H_{L}^{\omega}\right)\right\} \leq \sharp\left\{(-\infty,-1) \cap \sigma\left(H_{L}^{\omega, a}\right)\right\} .
$$

Let $\widetilde{H_{L+3}^{\omega, a}}$ be the operator obtained by replacing $A\left(x, \overline{W_{\omega}}(x)\right)$ and $W_{0}^{\omega}(x)^{n}$ by $\mathcal{A}^{\omega}$ and $\left(\mathcal{V}^{\omega}-a-1\right)_{-}$, respectively, in the definition of $\widetilde{H_{L+3}^{\omega}}$ in (2.1). Then, since $\sigma\left(H_{L}^{\omega, a}\right) \subset \sigma\left(\widetilde{H_{L+3}^{\omega, a}}\right)$, we have

$$
\sharp\left\{(-\infty,-1) \cap \sigma\left(H_{L}^{\omega, a}\right)\right\} \leq \sharp\left\{(-\infty,-1) \cap \sigma\left(\widetilde{H_{L+3}^{\omega, a}}\right)\right\} .
$$

Let $\widehat{H_{L+3}^{\omega,-}}$ be the operator obtained by replacing $A\left(x, \overline{W_{\omega}}(x)\right)$ by $\mathcal{A}^{\omega}$ in the definition of $\widehat{H_{L+3}^{\omega}}$ in $(2.2)$, and $\Gamma^{a}(\omega, L+3)$ be the operator obtained by replacing $\widehat{H_{L+3}^{\omega}}, E$ and $W_{0}^{\omega}(x)^{n}$ by $\widehat{H_{L+3}^{\omega,-}},-1$ and $\left(\mathcal{V}^{\omega}-a-1\right)_{-}$, respectively, in the definition of $\Gamma(\omega, L+3, E)$ in (2.6). Then, by the Birman-Schwinger principle (cf. Theorem 8.1 in [34]), we have

$$
\sharp\left\{(-\infty,-1) \cap \sigma\left(\widetilde{H_{L+3}^{\omega, a}}\right)\right\} \leq \sharp\left\{(1, \infty) \cap \sigma\left(\Gamma^{a}(\omega, L+3)\right)\right\} .
$$


This is less than or equal to

$$
\left\|\Gamma^{a}(\omega, L+3)\right\| \|_{q / 2}^{q / 2}
$$

By Lemma 2.1 (ii), we see this is dominated by

$$
\left\|\widetilde{\chi_{L+3}}\left(\mathcal{V}^{\omega}-a-1\right)_{-}\right\|_{q / 2}^{q / 2}
$$

By Lemma 3.1 (i), we have

$$
\begin{aligned}
E\left[\left\|\widetilde{\chi_{L+3}}\left(\mathcal{V}^{\omega}-a-1\right)_{-}\right\|_{q / 2}^{q / 2}\right] & \leq \sum_{b \in \Lambda_{L+3} \cap \mathbb{Z}^{d}} E\left[\operatorname{ess~sup}_{x \in \Lambda_{2}(b)}\left|\left(\mathcal{V}^{\omega}-a-1\right)_{-}\right|^{q / 2}\right] \\
& \leq C\left(\delta_{1}, \delta_{2}, \beta, a, q\right) \sharp\left\{\Lambda_{L+3} \cap \mathbb{Z}^{d}\right\} .
\end{aligned}
$$

Therefore we obtain (3.9).

The following ensures that the assumption (SGEE) in [14] relating a generalized eigenfunction expansion holds:

Proposition 3.5. For any $a<b$ and $p>0$, we have

$$
E\left[\operatorname{Tr}\left[T^{-1} E\left([a, b]: H^{\omega}\right) T^{-1}\right]^{p}\right]<\infty .
$$

The assumption (SGEE) in [14] requires (3.10) only for $p=2$. However we need (3.10) for some $p>2$ since our scalar potential $\mathcal{V}^{\omega}$ is not bounded below.

Proof of Proposition 3.5. By taking a complete orthonormal system $\left\{\varphi_{m}\right\}$ of $L^{2}\left(\mathbb{R}^{d}\right)$, we write the trace as

$$
\operatorname{Tr}\left[T^{-1} E\left([a, b]: H^{\omega}\right) T^{-1}\right]=\sum_{m}\left\|E\left([a, b]: H^{\omega}\right) T^{-1} \varphi_{m}\right\|_{2}^{2} .
$$

Since $\left(H^{\omega}-a+1\right)^{-1} \geq(b-a+1)^{-1}$ on the range of $E\left([a, b]: H^{\omega}\right)$, we have

$$
\begin{aligned}
& \left\|E\left([a, b]: H^{\omega}\right) T^{-1} \varphi_{m}\right\|_{2} \\
& \leq(b-a+1)\left\|\left(H^{\omega}-a+1\right)^{-1} E\left([a, b]: H^{\omega}\right) T^{-1} \varphi_{m}\right\|_{2} .
\end{aligned}
$$

Since

$$
\begin{gathered}
\left(H^{\omega}-a+1\right)^{-1} E\left([a, b]: H^{\omega}\right)=E\left([a, b]: H^{\omega}\right)\left(\widehat{H^{\omega,+}}+1\right)^{-1} \\
+\left(H^{\omega}-a+1\right)^{-1} E\left([a, b]: H^{\omega}\right)\left(a+\mathcal{V}_{-}^{\omega}\right)\left(\widehat{H^{\omega,+}}+1\right)^{-1}
\end{gathered}
$$


we have

$$
\begin{aligned}
& \left\|\left(H^{\omega}-a+1\right)^{-1} E\left([a, b]: H^{\omega}\right) T^{-1} \varphi_{m}\right\|_{2} \\
& \leq\left\|E\left([a, b]: H^{\omega}\right)\left(\widehat{H^{\omega,+}}+1\right)^{-1} T^{-1} \varphi_{m}\right\|_{2} \\
& \quad+\left\|\left(H^{\omega}-a+1\right)^{-1} E\left([a, b]: H^{\omega}\right)\left(a+\mathcal{V}_{-}^{\omega}\right)\left(\widehat{H^{\omega,+}}+1\right)^{-1} T^{-1} \varphi_{m}\right\|_{2},
\end{aligned}
$$

where $\widehat{H^{\omega,+}}=\left(i \nabla+\mathcal{A}^{\omega}\right)^{2}+\mathcal{V}_{+}^{\omega}$. The second term is less than of equal to

$$
\left\|\left(a+\mathcal{V}_{-}^{\omega}\right)\left(\widehat{H^{\omega,+}}+1\right)^{-1} T^{-1} \varphi_{m}\right\|_{2}
$$

since $\left(H^{\omega}-a+1\right)^{-1} \leq 1$ on the range of $E\left([a, b]: H^{\omega}\right)$. Therefore the quantity in (3.11) is dominated by

$$
\operatorname{Tr}\left[T^{-1}\left(\widehat{H^{\omega,+}}+1\right)^{-2} T^{-1}\right]+\operatorname{Tr}\left[T^{-1}\left(\widehat{H^{\omega,+}}+1\right)^{-1}\left(a+\mathcal{V}_{-}^{\omega}\right)^{2}\left(\widehat{H^{\omega,+}}+1\right)^{-1} T^{-1}\right] .
$$

By using the diamagnetic inequality as in the proof of Lemma 2.1, we see that this is less than or equal to

$$
\operatorname{Tr}\left[T^{-1}(-\Delta+1)^{-2} T^{-1}\right]+\operatorname{Tr}\left[T^{-1}(-\Delta+1)^{-1}\left(a+\mathcal{V}_{-}^{\omega}\right)^{2}(-\Delta+1)^{-1} T^{-1}\right] .
$$

By the explicit representation of the resolvent kernel we see that the first term is finite if $d<4$. By using also the boundedness of $E\left[\prod_{\iota=1}^{2 N}\left(a+\mathcal{V}_{-}^{\omega}\left(x_{\iota}\right)\right)^{2}\right]$, we see that

$$
E\left[\operatorname{Tr}\left[T^{-1}(-\Delta+1)^{-1}\left(a+\mathcal{V}_{-}^{\omega}\right)^{2}(-\Delta+1)^{-1} T^{-1}\right]^{2 N}\right]
$$

is finite for any $N \in \mathbb{N}$ if $d<4$. For the higher dimensional case, we take $\ell \in \mathbb{N}$ so that $\ell>d / 4$. By repeating the estimates as in (3.12) and (3.13), we have

$$
\begin{aligned}
& \left\|E\left([a, b]: H^{\omega}\right) T^{-1} \varphi_{m}\right\|_{2} \\
& \leq C \sum_{j_{1}, j_{2}, \ldots, j_{\ell} \in\{1,2\}} \|\left(a+\mathcal{V}_{-}^{\omega}\right)^{j_{1}}\left(\widehat{H^{\omega,+}}+1\right)^{-1}\left(a+\mathcal{V}_{-}^{\omega}\right)^{j_{2}}\left(\widehat{H^{\omega,+}}+1\right)^{-1} \cdots \\
& \quad \times\left(a+\mathcal{V}_{-}^{\omega}\right)^{j_{\ell}}\left(\widehat{H^{\omega,+}}+1\right)^{-1} T^{-1} \varphi_{m} \|_{2} .
\end{aligned}
$$

The rest of the proof is similar.

\section{§3.3. Proof of the main theorems}

Theorem 3.1 is proven by extending the four theorems in Section 5 in [14] and combining them as in [14]. The first one of them is extended as follows: 
Proposition 3.6 (cf. Theorem 5.1 in [14]). For any $E_{0} \in I_{0}, \theta>$ $b d / h$, odd integer $Y$ with $Y \geq 11$ and $0<p<h \theta-b d$, there exists $\mathcal{Z}_{\theta}=$ $\mathcal{Z}_{\theta}\left(d, \rho, M_{I_{0}}, Q_{\widetilde{I}_{0}}, \beta, \delta_{1}, \delta_{2}, b, h, \theta, p, Y\right) \in 6 \mathbb{N}$ satisfying the following: if there exists $\mathcal{Z}_{\theta} \leq L_{0} \in 6 \mathbb{N}$ such that

$$
P\left(\Lambda_{L_{0}} \text { is }\left(\theta, E_{0}\right) \text {-suitable }\right)>1-(3 Y-4)^{-2 d},
$$

then there exists $\kappa=\kappa\left(p, Y, L_{0}\right) \in \mathbb{Z}_{+}$such that

$$
P\left(\Lambda_{L_{k}} \text { is }\left(\theta, E_{0}\right) \text {-suitable }\right)>1-L_{k}^{-p}
$$

for any $k \geq \kappa$, where $L_{k+1}=Y L_{k}$ for any $k \in \mathbb{Z}_{+}$.

Proof. In the proof of Theorem 5.1 in [14], we require $s$ satisfies

$$
(p+b d) / h<s \text { and } s<\theta .
$$

As in [14], we set

$$
\begin{aligned}
& q_{L}=L^{-p}, t_{L}=L^{-s}, u_{L}=L^{-\theta} \\
& p_{L}=P\left(\Lambda_{L} \text { is not }\left(\theta, E_{0}\right) \text {-suitable }\right)
\end{aligned}
$$

Let $\ell \in 6 \mathbb{N}, \ell>6 \rho, Y \in 2 \mathbb{N}+1$ and $L=Y \ell$. We set

$$
\Xi_{L, \ell}=\Lambda_{L} \cap \frac{\ell}{3} \mathbb{Z}^{d}, \quad C_{L, \ell}=\left\{\Lambda_{\ell}(y): y \in \Xi_{L, \ell}, \Lambda_{\ell}(y) \sqsubset \Lambda_{L}\right\} .
$$

In the definition of the event $\mathcal{F}_{L, \ell}$ in [14], we add the conditions on $\mathcal{V}_{-}^{\omega}$ as follows:

$$
\begin{aligned}
\mathcal{F}_{L, \ell}=\{\omega: & \text { there exist two }\left(\theta, E_{0}\right) \text {-nonsuitable boxes } \\
& \Lambda_{\ell_{1}}\left(y_{1}\right), \Lambda_{\ell_{2}}\left(y_{2}\right) \text { in } C_{L, \ell} \text { such that } \\
& \left.\operatorname{dist}\left(\Lambda_{\ell_{1}}\left(y_{1}\right), \Lambda_{\ell_{2}}\left(y_{2}\right)\right)>2 \rho\right\} \\
\cup & \left\{\omega: \operatorname{dist}\left(\sigma\left(H_{x, 3 \ell}^{\omega}\right), E_{0}\right) \leq t_{L} \text { for some } x \in \Xi_{L, \ell}^{\prime}\right\} \\
\cup & \left\{\omega: \operatorname{dist}\left(\sigma\left(H_{0, L}^{\omega}\right), E_{0}\right) \leq t_{L}\right\} \\
\cup & \left\{\omega: \operatorname{ess} \sup \mathcal{V}_{-}^{\omega} \geq\left\{2 p(\log L)^{2} / \delta_{2}\right\}^{1 / \beta}\right\} .
\end{aligned}
$$

By Lemma 3.2, we have

$$
\left\{\omega: \Lambda_{L} \text { is not }\left(\theta, E_{0}\right) \text {-suitable }\right\} \subset \mathcal{F}_{L, \ell}
$$


for $L>\mathcal{Z}_{1}=\mathcal{Z}_{1}\left(d, \beta, \delta_{1}, \delta_{2}, \theta, p, Y, s\right)$. By the assumptions (W), (F) and Lemma 3.4 below, we have

$$
\begin{aligned}
P\left(\mathcal{F}_{L, \ell}\right) & \leq \frac{(3 Y-4)^{2 d}}{2} p_{\ell}^{2}+\left\{(6 Y)^{d}+1\right\} Q_{\widetilde{I}_{0}} L^{b d} t_{L}^{h}+\delta_{1} L^{-2 p} \\
& \leq \frac{(3 Y-4)^{2 d}}{2} p_{\ell}^{2}+\frac{1}{2} q_{L}
\end{aligned}
$$

for $L>\mathcal{Z}_{2}=\mathcal{Z}_{2}\left(d, Q_{\widetilde{I_{0}}}, \beta, \delta_{1}, \delta_{2}, b, h, \theta, p, Y, s\right)$. The rest of the proof is same.

Lemma 3.4. Let $\Lambda_{\ell_{1}}\left(y_{1}\right), \Lambda_{\ell_{2}}\left(y_{2}\right), \ldots, \Lambda_{\ell_{m}}\left(y_{m}\right)$ be finite number of boxes such that $\operatorname{dist}\left(\Lambda_{\ell_{i}}\left(y_{i}\right), \Lambda_{\ell_{j}}\left(y_{j}\right)\right)>2 \rho$ for $i \neq j$. Then, for any $\theta$ and $E$, the events $\left\{\omega: \Lambda_{\ell_{i}}\left(y_{i}\right)\right.$ is $(\theta, E)$-suitable $\}, i=1,2, \ldots, m$, are independent.

Proof. Let $\zeta_{i} \in C_{0}^{\infty}\left(\mathbb{R}^{d} \rightarrow[0,1]\right)$ such that $\zeta_{i} \equiv 1$ on $\Lambda_{\ell_{i}}\left(y_{i}\right)$ and $\zeta_{i} \equiv 0$ on $\Lambda_{\ell_{i}+\rho / 2}\left(y_{i}\right)^{c}$. We define

$$
\mathcal{A}_{j}^{[i], \omega}(x):=\frac{-\Gamma(d / 2)}{2 \pi^{d / 2}} \int \sum_{k \neq j} \frac{x_{k}-y_{k}}{|x-y|^{d}} d\left(\zeta_{i} \mathcal{A}^{(2), \omega}\right)_{j, k}(y) \zeta_{i}(y) d y .
$$

Since $\mathcal{A}^{(2), \omega}$ and $d \mathcal{A}^{(2), \omega}$ are continuous in $x, \mathcal{A}^{[i], \omega}$ is also continuous in $x$ and we can integrate $\mathcal{A}^{(2), \omega}$ and $\mathcal{A}^{[i], \omega}$ along any $C^{1}$ curves. Since $d \mathcal{A}^{[i], \omega}=d \mathcal{A}^{(2), \omega}$ on $\Lambda_{\ell_{i}}\left(y_{i}\right)$, we can construct $\Phi^{[i], \omega} \in C^{1}\left(\Lambda_{\ell_{i}}\left(y_{i}\right)\right)$ such that $d \Phi^{[i], \omega}=\mathcal{A}^{(2), \omega}-$ $\mathcal{A}^{[i], \omega}$ by the Stokes theorem. By the unitary operator $\exp \left(i \Phi^{[i], \omega}\right)$, the operators $H_{\ell_{i}, y_{i}}^{\omega}$ and $H_{\ell_{i}, y_{i}}^{[i], \omega}$ are unitary equivalent, where $H_{\ell_{i}, y_{i}}^{[i], \omega}$ is the operator obtained by replacing $\mathcal{A}^{(2), \omega}$ by $\mathcal{A}^{[i], \omega}$ in the definition of $H_{\ell_{i}, y_{i}}^{\omega}$. Since the coefficients of $H_{\ell_{i}, y_{i}}^{[i], \omega}$ are determined by the random field $\left\{\left(\mathcal{A}^{(1), \omega}, d \mathcal{A}^{(2), \omega}, \mathcal{V}^{\omega}(x)\right)\right.$ : $\left.x \in \Lambda_{\ell_{i}+\rho / 2}\left(y_{i}\right)\right\}$, the desired independence follows from the assumption (IAD).

The rest of the theorems in Section 5 in [14] are also modified by using the assumptions (F) and (IAD) as above. We omit the detail.

Theorems 3.2 and 3.3 are proven similarly as in [14] by using Lemma 3.1 and the propositions in the last subsection.

Proposition 3.1 is proven by using the subsolution estimate (Theorem 8.17 in [19]) as in [28]. For this, we use Kato's inequality (Theorem X.33 in [32]) to show $\left(-\Delta+\left(\mathcal{V}^{\omega}-E_{j, \omega}\right)_{-}\right)\left|\phi_{j, \omega}(x)\right| \leq 0$ in the weak sense. 


\section{§3.4. The case of Section 2}

In the case of Section 2, the assumptions (R), (S) and (IAD) hold. Moreover the assumption $(\mathrm{K})$ in Proposition 3.1 is also satisfied. For any open interval $\widetilde{I_{0}}$ such that $\left.\sup \widetilde{I}_{0}<-\left(2\left\|w \cdot \nabla_{w} A\right\|_{\infty} / n\right)^{2}\right)$, the assumption (W) with $\widetilde{I_{0}}$ is ensured by Theorem 2.1. The assumption $(\mathrm{F})$ is ensured by the following lemma by Fischer, Leschke and Müller [12], which is based on Fernique's theorem [10]:

Lemma 3.5 (Lemma 5.3 in [12]). $\quad$ There exists a positive finite constant $L_{0}=L_{0}\left(R^{00}(0),\left\|\nabla R^{00}\right\|_{\infty}\right)$ such that

$$
P\left(\sup _{x \in \Lambda_{L}}\left|W_{\omega}^{0}(x)\right| \geq \eta\right) \leq 2^{2(d+1)} \exp \left(-\frac{\eta^{2}}{200 R^{00}(0) \log L}\right)
$$

for any $L \geq L_{0}$ and $\eta \geq 0$.

The initial estimate (3.3) is also proven by using this lemma and a CombesThomas estimate, Lemma A.1 in [12] as in Lemma 5.5 in [12]:

Lemma 3.6. There exist positive constants $c_{1}=c_{1}(d), c_{2}=c_{2}(d)$ and a universal finite constant $c_{3}$ such that for any $\theta>0,12 \leq L \in 6 \mathbb{N}$ and $E \leq-\mathfrak{E}(L)$, we have

$$
P\left(\Lambda_{L} \text { is }(\theta, E) \text {-suitable }\right)>1-841^{-d},
$$

where

$$
\mathfrak{E}(L):=\left\{c_{3} L^{-1} \log \left(c_{1} L^{\theta+d+1}\right)\right\}^{2}+\left(c_{2} R^{00}(0) \log L\right)^{n / 2} .
$$

Therefore the results in Subsection 3.1 hold for

$$
E_{0}<\left\{-\left(2\left\|w \cdot \nabla_{w} A\right\|_{\infty} / n\right)^{2}\right\} \wedge\left\{-\inf _{\theta>b d / h} \mathfrak{E}\left(\overline{L_{\theta}}\right)\right\},
$$

where $\overline{L_{\theta}}$ is the constant given in Theorem 3.1 .

These results are meaningful since the interval determined by (3.15) is included in the spectrum as is shown in the following:

Proposition 3.7 (Theorem 5.34 in [31]). We have

$$
\begin{aligned}
\sigma\left(H^{\omega}\right) \supset\left\{\inf \sigma(H(\ell, \bar{w}))-\left(w^{0}\right)^{n}\right. \\
\left.\quad: \ell>0, w^{0} \in \mathbb{R}, \bar{w} \in \operatorname{supp} P\left(\overline{W_{\omega}}(0) \in \cdot \mid W_{\omega}^{0}(0)=w^{0}\right)\right\}
\end{aligned}
$$


for a.e. $\omega$, where $H(\ell, \omega)$ is the self-adjoint operator on $L^{2}\left(\Lambda_{\ell}\right)$ obtained by restricting

$$
\sum_{j=1}^{d}\left(i \partial_{j}+A_{j}(x, \bar{w})\right)^{2}
$$

to $\Lambda_{\ell}$ by the Dirichlet boundary condition. In particular, if

$$
\sup \left\{|A(x, \bar{w})|: x \in \Lambda_{\ell}, \bar{w} \in \mathbb{R}^{g}\right\}<\infty
$$

for any $\ell>0$, then we have

$$
\sigma\left(H^{\omega}\right)=\mathbb{R}
$$

for a.e. $\omega$.

Proof. For any $w^{0}>0, \bar{w} \in \operatorname{supp} P\left(\overline{W_{\omega}}(0) \in \cdot \mid W_{\omega}^{0}(0)=w^{0}\right), \ell>0$ and $\left|w^{0}\right|>\varepsilon>0$, we have

$$
P\left(\sup _{x \in \Lambda_{\ell}}\left|\left(\overline{W_{\omega}}(x), W_{\omega}^{0}(x)\right)-\left(\bar{w}, w^{0}\right)\right|<\varepsilon\right)>0 .
$$

If the event in this probability occurs, then by using

$$
\left|A\left(x, \overline{W_{\omega}}(x)\right)-A(x, \bar{w})\right| \leq \varepsilon\left\|\nabla_{\bar{w}} A\right\|_{\infty},
$$

we have

$$
\inf \sigma\left(H_{0, \ell}^{\omega}\right) \leq(1+\varepsilon) \inf \sigma(H(\bar{w}, \ell))+\varepsilon(1+\varepsilon)\left\|\nabla_{\bar{w}} A\right\|_{\infty}^{2}-\left(w^{0}-\varepsilon\right)^{n}=: \overline{\mathcal{W}}(\varepsilon)
$$

and

$$
\inf \sigma\left(H_{0, \ell}^{\omega}\right) \geq(1-\varepsilon) \inf \sigma(H(\bar{w}, \ell))-\varepsilon(1-\varepsilon)\left\|\nabla_{\bar{w}} A\right\|_{\infty}^{2}-\left(w^{0}+\varepsilon\right)^{n}=: \underline{\mathcal{W}}(\varepsilon)
$$

Since $\inf \sigma\left(H_{0, \ell}^{\omega}\right) \subset \sigma\left(H^{\omega}\right)$, we have

$$
P\left((\underline{\mathcal{W}}(\varepsilon), \overline{\mathcal{W}}(\varepsilon)) \cap \sigma\left(H^{\omega}\right) \neq \emptyset\right)>0 .
$$

By the ergodicity, this probability is one. Therefore we obtain

$$
\begin{aligned}
1 & =P\left((\underline{\mathcal{W}}(1 / n), \overline{\mathcal{W}}(1 / n)) \cap \sigma\left(H^{\omega}\right) \neq \emptyset \text { for any } 1 / w_{0}<n \in \mathbb{N}\right) \\
& =P\left(\inf \sigma(H(\bar{w}, \ell))-\left(w^{0}\right)^{n} \in \sigma\left(H^{\omega}\right)\right) .
\end{aligned}
$$

By the same method we can prove this for $w^{0} \leq 0$. 
We next take an $\mathbb{R}^{g}$-valued continuous function $\bar{w}\left(w^{0}\right)$ of $w^{0} \in \mathbb{R}$ so that $\bar{w}\left(w^{0}\right) \in \operatorname{supp} P\left(\overline{W_{\omega}}(0) \in \cdot \mid W_{\omega}^{0}(0)=w^{0}\right)$ and $\bar{w}(0)=0$. Then $s\left(w^{0}, \ell\right):=$ $\inf \sigma\left(H\left(\bar{w}\left(w^{0}\right), \ell\right)\right)-\left(w^{0}\right)^{n}$ is continuous in $w_{0}$. If

$$
\alpha_{\ell}:=\sup \left\{|A(x, \bar{w})|: x \in \Lambda_{\ell}, \bar{w} \in \mathbb{R}^{g}\right\}<\infty
$$

for any $\ell>0$, then

$$
s\left(w^{0}, \ell\right) \leq 2 \inf \sigma\left(-\Delta_{\ell}\right)+2 \alpha_{\ell}^{2}-\left(w^{0}\right)^{n},
$$

where $\Delta_{\ell}$ is the Dirichlet Laplacian on $\Lambda_{\ell}$. By this we see that $s\left(w^{0}, \ell\right)$ tends to $-\infty$ as $w^{0}$ tends to $\infty$. Therefore we have $(-\infty, s(0, \ell)) \subset \sigma\left(H^{\omega}\right)$. By the diamagnetic inequality we have

$$
s(0, \ell) \geq \inf \sigma\left(-\Delta_{\ell}\right) .
$$

Then we see that $s(0, \ell)$ tends to $\infty$ as $\ell$ tends to 0 . Therefore we have $\sigma\left(H^{\omega}\right)=$ $\mathbb{R}$.

Remark 3.1. In the case that $A_{j}$ is independent of $w$ and $n=1$, Fischer, Leschke and Müller [12] proves the absence of the continuous spectrum in a low energy interval under more general conditions on the Gaussian random field.

Remark 3.2. The results in this subsection are extended to the cases stated in Remark 2.2.

\section{§4. A Characterization of the Anderson Metal-insulator Transport Transition}

In this section we extend the theory in Germinet and Klein [16]. As in the last section, we treat a random operator (3.1) with a general random field $\left(\mathcal{A}^{\omega}(x), \mathcal{V}^{\omega}(x)\right)$. In $[16], \mathcal{A}^{\omega}=0, \mathcal{V}^{\omega}$ is relatively form bounded below with respect to the negative Laplacian and the Wegner estimate (W) holds with $h=b=1$. In this section we remove this restrictions so that the operator in the preceding sections can be treated.

\section{§4.1. Main theorem}

We use the notations in the last section. We assume (R), (IAD), (F) and $(\mathrm{W})$ on an open interval $\widetilde{I_{0}}$. Moreover we assume the ergodicity:

(E) The random field $\left(\mathcal{A}^{(1), \omega}(x), d \mathcal{A}^{(2), \omega}(x), \mathcal{V}^{\omega}(x)\right)$ is stationary and ergodic, with respect to the shift in the variable $x \in \mathbb{R}^{d}$ or $\mathbb{Z}^{d}$. 
Then we have a nonrandom set $\Sigma$ such that $\sigma\left(H^{\omega}\right)=\Sigma$ with probability one (cf. [31], [38]). Referring Theorem 3.1, we modify the definition of the multiscale analysis region $\Sigma_{M S A}$ in [16] as

$$
\begin{aligned}
& \Sigma_{M S A}:=\left\{E \in \Sigma: \text { there exist } \theta>b d / h, \text { a compact interval } I_{0}\right. \\
& \text { and } \mathcal{L} \in 6 \mathbb{N} \text { such that } E \in \stackrel{\circ}{I}_{0} \subset I_{0} \subset \widetilde{I_{0}}, \mathcal{L} \geq \overline{\mathcal{L}_{\theta}} \text { and } \\
& P\left(\Lambda_{L}:(\theta, E) \text { - suitable }\right)>1-841^{-d}, \\
&\text { where } \left.\overline{\mathcal{L}_{\theta}} \text { is the number given in Theorem } 3.1\right\} .
\end{aligned}
$$

As in [16], we consider moments defined as follows: for any $n \geq 0, f \in C_{0}^{\infty}(\mathbb{R} \rightarrow$ $[0, \infty)), t \in \mathbb{R}$ and $T \geq 0$, we set

$$
\begin{aligned}
M_{\omega}(n, f, t) & :=\|\|\langle X\rangle^{n / 2} \exp \left(-i t H^{\omega}\right) f\left(H^{\omega}\right) \chi_{0} \|\left.\right|_{2} ^{2} \\
\mathbb{M}(n, f, t) & :=E\left[M_{\omega}(n, f, t)\right]
\end{aligned}
$$

and

$$
\mathcal{M}(n, f, T):=\frac{2}{T} \int_{0}^{\infty} e^{-2 t / T} \mathbb{M}(n, f, t) d t,
$$

where $\langle X\rangle:=\left(1+|X|^{2}\right)^{1 / 2}$. The strong insulator region $\Sigma_{S I}$ is defined by

$$
\begin{aligned}
& \Sigma_{S I}:=\{E \in \Sigma: \text { there exists an open interval } I \text { including } E \\
& \text { such that } E\left[\sup _{t \in \mathbb{R}} M_{\omega}(n, f, t)\right]<\infty \\
&\text { for any } \left.f \in C_{0}^{\infty}(I \rightarrow[0, \infty)) \text { and } n \geq 0\right\}
\end{aligned}
$$

By Theorem 3.2, we have $\Sigma_{M S A} \cap \widetilde{I}_{0} \subset \Sigma_{S I}$. Moreover, if $E \in \Sigma_{S I}$ and $I$ is an open interval appeared in the definition of $E \in \Sigma_{S I}$, then it is easy to see that

$$
\sup _{T \geq 0} \mathcal{M}(n, f, T)<\infty
$$

for any $n \geq 0$ and $f \in C_{0}^{\infty}(I \rightarrow[0, \infty))$. Conversely, we have the following, which is an extension of Theorem 2.11 in [16]:

Theorem 4.1. Let $f \in C_{0}^{\infty}(\mathbb{R} \rightarrow[0, \infty))$ such that $f \equiv 1$ on some open interval $J \subset \widetilde{I_{0}}, \alpha \geq 0$ and

$$
n>2 b d \alpha / h+(2+9 b / h) d
$$


If

$$
\varliminf_{T \rightarrow \infty} \mathcal{M}(n, f, T) / T^{\alpha}<\infty
$$

then $J \cap \Sigma \subset \Sigma_{M S A}$.

We prove this theorem in the next subsection. In [16], many results are deduced from this result. Some of the results are stated in terms of an exponent defined in (4.9) below. To define the exponent in our case, we extend Proposition 3.1 in [16] as follows:

Proposition 4.1. Let $f \in C_{0}^{\infty}(\mathbb{R} \rightarrow[0, \infty))$ such that $f\left(H^{\omega}\right) \neq 0$ with probability one. Then we have

$$
\begin{aligned}
& 0 \leq M_{\omega}(0, f, 0) \leq M_{\omega}(n, f, t) \leq C(d, \beta, f, n, \omega)\langle t\rangle^{[[n+(5 d+1) / 2]]+2} \\
& 0<\mathbb{M}(0, f, 0) \leq \mathbb{M}(n, f, t) \leq C(d, \beta, f, n)\langle t\rangle^{[[n+(5 d+1) / 2]]+2}
\end{aligned}
$$

and

$$
0<\mathbb{M}(0, f, 0) \leq \mathcal{M}(n, f, T) \leq C^{\prime}(d, \beta, f, n)\langle T\rangle^{[[n+(5 d+1) / 2]]+2}
$$

for any $t \in \mathbb{R}$ and $n, T \geq 0$, where $[[m]]$ is the smallest integer greater than $m$ for any $m \geq 0$.

We prove this proposition in Subsection 4.3 below. In the situation of Proposition 3.1 in [16], the exponents of $\langle t\rangle$ and $\langle T\rangle$ in the right hand sides of $(4.5)-(4.7)$ are taken as $[n+3 d / 2]+3$ and the constant $C(d, \beta, f, n, \omega)$ in (4.5) is independent of $\omega$.

As in [16] we define the $n$-th upper and lower transport exponents as follows: for $f \in C_{0}^{\infty}(\mathbb{R} \rightarrow[0, \infty))$ with $f\left(H^{\omega}\right) \neq 0$ with probability one, we set

$$
\beta^{+}(n, f):=\varlimsup_{T \rightarrow \infty} \frac{\log \mathcal{M}(n, f, T)}{n \log T}
$$

and

$$
\beta^{-}(n, f):=\varliminf_{T \rightarrow \infty} \frac{\log \mathcal{M}(n, f, T)}{n \log T} .
$$

For $f \in C_{0}^{\infty}(\mathbb{R} \rightarrow[0, \infty))$ with $f\left(H^{\omega}\right) \neq 0$ with probability one, we set $\beta^{ \pm}(n, f)=0$. For any open interval $I$ and $E \in \mathbb{R}$, we set

$$
\beta^{ \pm}(n, I):=\sup \left\{\beta^{ \pm}(n, f) \mid f \in C_{0}^{\infty}(I \rightarrow[0, \infty))\right\}
$$

and

$$
\beta^{ \pm}(n, E):=\inf \left\{\beta^{ \pm}(n, I) \mid I \text { is an open interval including } E\right\} .
$$

For these exponents we obtain the following by the entirely same proof of Proposition 3.2 in [16]: 
Proposition 4.2. For any $f \in C_{0}^{\infty}(\mathbb{R} \rightarrow[0, \infty))$, any open interval $I$ and any $E \in \mathbb{R}$, we have the following:

(i) $\beta^{ \pm}(n, f), \beta^{ \pm}(n, I)$ and $\beta^{ \pm}(n, E)$ are monotone increasing in $n>0$.

(ii) $0 \leq \beta^{ \pm}(n, f), \beta^{ \pm}(n, I), \beta^{ \pm}(n, E) \leq 1$.

Therefore, as in [16], we can define

$$
\begin{aligned}
& \beta^{ \pm}(I)=\lim _{n \rightarrow \infty} \beta^{ \pm}(n, I)=\sup _{n} \beta^{ \pm}(n, I) \\
& \beta^{ \pm}(E)=\lim _{n \rightarrow \infty} \beta^{ \pm}(n, E)=\sup _{n} \beta^{ \pm}(n, E)
\end{aligned}
$$

and we have $0 \leq \beta^{ \pm}(I), \beta^{ \pm}(E) \leq 1$ and $\beta^{ \pm}(E)=0$ for $E \notin \Sigma$.

From Theorem 4.1 we have the following as in Theorem 2.10 in [16]:

Proposition 4.3. If $E \in \widetilde{I}_{0}$ and $\beta^{-}(E)>0$, then $\beta^{-}(n, E) \geq h /(2 b d)-$ $(2 h+9 b) /(2 n b)$ for all $n>(2 h+9 b) d / h$ and $\beta^{-}(E) \geq h /(2 b d)$.

Therefore the size of the jump of the exponent $\beta^{-}$at the transition point is greater than or equal to $h /(2 b d)$.

Theorems 2.8 and 4.2 in [16] are also extended appropriately.

\section{§4.2. Proof of Theorem 4.1}

To prove Theorem 4.1, we first modify Theorem A.5 in [16] (Theorem 2 in [15]) as follows:

Proposition 4.4. There exist finite constants $C(d)$ and $C$ such that

$$
\begin{aligned}
& \left\|\chi_{x} f\left(H^{\omega}\right) \chi_{y} \mid\right\| \\
& \leq C(d)\|f\|_{(k+1)}\left(\underset{\substack{\operatorname{ess} \sup \\
\Lambda_{R L}}}{\leq}+1\right)^{(2 k+d-3) / 4} \frac{(C k)^{k}}{\langle x-y\rangle^{(2 k-d-3) / 2}}
\end{aligned}
$$

for any $f \in C^{\infty}(\mathbb{R} \rightarrow \mathbb{C}),(d+5) / 2 \leq k \in \mathbb{N}, L \geq 2$ and $x, y \in \Lambda_{L-1}$, where $R=2 \sqrt{d}+4$ and

$$
\|f\|_{(n)}:=\sum_{r=0}^{n} \int_{\mathbb{R}}\left|f^{(r)}(u)\right|\langle u\rangle^{r-1} d u
$$

for $n \in \mathbb{N}$.

The number $R=2 \sqrt{d}+4$ can be replaced by any number greater than $2 \sqrt{d}+2$ if $L$ is large enough.

To prove this proposition, we first show the following: 
Lemma 4.1. $\quad$ There is a finite constant $C(d)$ such that

$$
\begin{aligned}
& \left\|\left|\chi_{x}\left(H^{\omega}-z\right)^{-1} \chi_{y}\right|\right\| \mid \\
& \leq \frac{C(d)}{\eta_{z}^{R L}}\left\{1+\left(\left(\eta_{z}+|z|+\underset{\Lambda_{R L}}{\operatorname{ess} \sup } \mathcal{V}_{-}^{\omega}+1\right)^{1 / 2} / \eta_{z}\right)\right. \\
& \left.\quad \times\left(\left(\eta_{z}^{R L}+|z|+\underset{\Lambda_{R L}}{\operatorname{ess} \sup } \mathcal{V}_{-}^{\omega}+1\right) / \eta_{z}^{R L}\right)^{(d-1) / 2}\right\} \\
& \quad \times \exp \left(-\frac{m_{z}^{R L}}{2+\sqrt{\eta_{z}^{R L}}}|x-y|\right)
\end{aligned}
$$

for any $z \in \mathbb{C}-\mathbb{R}, L \geq 2$ and $x, y \in \Lambda_{L-1}$, where $\eta_{z}:=\operatorname{dist}\left(z, \sigma\left(H^{\omega}\right)\right)$, $\eta_{z}^{R L}:=\operatorname{dist}\left(z, \sigma\left(H_{R L}^{\omega}\right)\right)$ and

$$
m_{z}^{R L}:=\frac{\eta_{z}^{R L}}{32}\left(\frac{63}{{\operatorname{ess} \sup _{\Lambda_{R L}} \mathcal{V}_{-}^{\omega}+\eta_{z}^{R L}+|z|}^{1 / 2}}\right)^{1}
$$

Proof. We take $\phi \in C_{0}^{\infty}\left(\Lambda_{R L} \rightarrow[0,1]\right)$ so that $\phi \equiv 1$ on $\Lambda_{L}$ and $\operatorname{supp} \nabla \phi$ $\subset \Lambda_{2(\sqrt{d}+1) L+3}-\Lambda_{2(\sqrt{d}+1) L+2}$. Then we have

$$
\begin{aligned}
\phi & \left(H_{R L}^{\omega}-z\right)^{-1}-\left(H^{\omega}-z\right)^{-1} \phi \\
= & 2\left(H^{\omega}-z\right)^{-1}(i \nabla \phi)\left(i \nabla+\mathcal{A}^{\omega}\right)\left(H_{R L}^{\omega}-z\right)^{-1} \\
& -\left(H^{\omega}-z\right)^{-1}(\Delta \phi)\left(H_{R L}^{\omega}-z\right)^{-1}
\end{aligned}
$$

and

$$
\begin{aligned}
& \|\| \chi_{x}\left(H^{\omega}-z\right)^{-1} \chi_{y}\left\|\left|\leq\left\|\mid \chi_{x}\left(H_{R L}^{\omega}-z\right)^{-1} \chi_{y}\right\| \|\right.\right. \\
& +\left(2\left\|\mid \chi_{x}\left(H^{\omega}-z\right)^{-1}(i \nabla \phi)\left(i \nabla+\mathcal{A}^{\omega}\right) \psi\right\| \|\right. \\
& \left.\quad+\|\| \chi_{x}\left(H^{\omega}-z\right)^{-1}(\Delta \phi) \|||\right)\left\|\psi \psi\left(H_{R L}^{\omega}-z\right)^{-1} \chi_{y}\right\| \|,
\end{aligned}
$$

where $\psi \in C_{0}^{\infty}\left(\Lambda_{R L} \rightarrow[0,1]\right)$ such that $\psi \equiv 1$ on $\Lambda_{2(\sqrt{d}+1) L+3}-\Lambda_{2(\sqrt{d}+1) L+2}$ and $\psi \equiv 0$ on $\left(\Lambda_{2(\sqrt{d}+1) L+4}-\Lambda_{2(\sqrt{d}+1) L+1}\right)^{c}$. By the same proof of Corollary 1 of Theorem 1 in [15], we have

$$
||\left|\chi_{x_{0}}\left(H_{R L}^{\omega}-z\right)^{-1} \chi_{y_{0}}\right||| \leq \frac{9}{\eta_{z}^{R L}} \exp \left(C \sqrt{d}-\frac{m_{z}^{R L}}{2+\sqrt{\eta_{z}^{R L}}}\left|x_{0}-y_{0}\right|\right)
$$


for some finite constant $C$ and any $x_{0}, y_{0} \in \Lambda_{R L-1}$. Since $\left|x_{0}-y\right| \geq(\sqrt{d}+1) L$ for any $x_{0} \notin \Lambda_{2(\sqrt{d}+1) L+1}$ and $y \in \Lambda_{L-1}$, we have

$$
\begin{aligned}
& \left\|\left|\psi\left(H_{R L}^{\omega}-z\right)^{-1} \chi_{y}\right|\right\| \mid \\
& \leq\left\{\left.\sum_{x_{0} \in\left(\Lambda_{2(\sqrt{d}+1) L+4}-\Lambda_{2(\sqrt{d}+1) L+1}\right) \cap \mathbb{Z}^{d}}\left\|\chi_{x_{0}}\left(H_{R L}^{\omega}-z\right)^{-1} \chi_{y}\right\|\right|^{2}\right\}^{1 / 2} \\
& \leq \frac{C^{\prime \prime}(d)}{\eta_{z}^{R L}} L^{(d-1) / 2} \exp \left(-\frac{m_{z}^{R L}(\sqrt{d}+1 / 2) L}{2+\sqrt{\eta_{z}^{R L}}}\right) \\
& \leq \frac{C^{\prime}(d)}{\eta_{z}^{R L}}\left(\frac{2+\sqrt{\eta_{z}^{R L}}}{m_{z}^{R L}}\right)^{(d-1) / 2} \exp \left(-\frac{m_{z}^{R L} \sqrt{d} L}{\left.2+\sqrt{\eta_{z}^{R L}}\right)}\right. \\
& \leq \frac{C(d)}{\eta_{z}^{R L}}\left\{\left(\eta_{z}^{R L}+|z|+\operatorname{ess} \sup \mathcal{V}_{-}^{\omega}+1\right) / \eta_{z}^{R L}\right\}^{(d-1) / 2} \\
& \quad \times \exp \left(-\frac{m_{z}^{R L} \sqrt{d} L}{2+\sqrt{\eta_{z}^{R L}}}\right) .
\end{aligned}
$$

Since $|x-y| \leq \sqrt{d} L$ for any $x, y \in \Lambda_{L-1}$, we have

$$
\exp \left(-\frac{m_{z}^{R L} \sqrt{d} L}{2+\sqrt{\eta_{z}^{R L}}}\right) \leq \exp \left(-\frac{m_{z}^{R L}}{2+\sqrt{\eta_{z}^{R L}}}|x-y|\right) .
$$

On the other hand we have

$$
\left\|\left|\psi\left(i \nabla+\mathcal{A}^{\omega}\right)\left(H^{\omega}-z\right)^{-1}\right|\right\| \leq \frac{C}{\eta_{z}}\left(\eta_{z}+|z|+\underset{\Lambda_{R L}}{\operatorname{ess} \sup } \mathcal{V}_{-}^{\omega}+1\right)^{1 / 2}
$$

for some finite constant $C$. In fact, for any $\phi \in C_{0}^{\infty}\left(\mathbb{R}^{d}\right)$, we have

$$
\begin{aligned}
& \left\|\psi\left(i \nabla+\mathcal{A}^{\omega}\right)\left(H^{\omega}-z\right)^{-1} \phi\right\|^{2} \\
& =\left(\left(H^{\omega}-z\right)^{-1} \phi,\left(i \nabla+\mathcal{A}^{\omega}\right) \psi^{2}\left(i \nabla+\mathcal{A}^{\omega}\right)\left(H^{\omega}-z\right)^{-1} \phi\right) .
\end{aligned}
$$

Since

$$
\begin{aligned}
& \left(i \nabla+\mathcal{A}^{\omega}\right) \psi^{2}\left(i \nabla+\mathcal{A}^{\omega}\right) \\
& =\psi^{2}\left(H^{\omega}-z\right)+\psi^{2}\left(z-\mathcal{V}^{\omega}\right)+2 \psi(i \nabla \psi)\left(i \nabla+\mathcal{A}^{\omega}\right),
\end{aligned}
$$

and $\|\left|\left(H^{\omega}-z\right)^{-1}\right| \mid \leq 1 / \eta_{z}$, we have

$$
\begin{aligned}
& \left\|\psi\left(i \nabla+\mathcal{A}^{\omega}\right)\left(H^{\omega}-z\right)^{-1} \phi\right\|^{2} \\
& \leq \frac{1}{\eta_{z}}\left\{1+\frac{1}{\eta_{z}}\left(|z|+\underset{\Lambda_{R L}}{\operatorname{ess} \sup } \mathcal{V}_{-}^{\omega}+\frac{1}{s} \sup |\nabla \psi|^{2}\right)\right\}\|\phi\|^{2} \\
& \quad+s\left\|\psi\left(i \nabla+\mathcal{A}^{\omega}\right)\left(H^{\omega}-z\right)^{-1} \phi\right\|^{2}
\end{aligned}
$$


for any $s>0$. By taking $s<1$, we obtain (4.15).

By applying (4.13), (4.15), (4.15), $\left\|\left|\left(H^{\omega}-z\right)^{-1}\right|\right\| \leq 1 / \eta_{z}$ and $\| \mid\left(H_{R L}^{\omega}-\right.$ $z)^{-1} \mid \| \leq 1 / \eta_{z}^{R L}$ to the right hand side of (4.12), we obtain (4.11).

Proof of Proposition 4.4. As in the proof of Theorem 2 in [15], we use the Helffer-Sjöstrand formula (cf. [8] Section 2.2):

$$
f\left(H^{\omega}\right)=\frac{1}{\pi} \int_{\mathbb{R}^{2}} \frac{\partial \tilde{f}_{k}(z)}{\partial \bar{z}}\left(H^{\omega}-z\right)^{-1} d u d v
$$

where $z=u+i v, k \in \mathbb{N}$,

$$
\widetilde{f}_{k}(z)=\left\{\sum_{r=0}^{k} \frac{1}{r !} f^{(r)}(u)(i v)^{r}\right\} \sigma(u, v),
$$

$\sigma(u, v)=\tau(v /\langle u\rangle)$ and $\tau \in C^{\infty}(\mathbb{R} \rightarrow[0,1])$ such that $\tau(s)=1$ on $|s| \leq 1$ and $\tau(s)=0$ on $|s| \geq 2$. Then we have

$$
\left\|\left|\chi_{x} f\left(H^{\omega}\right) \chi_{y} \|\right| \leq C\left(I_{1}+I_{2}\right),\right.
$$

where

$$
I_{1}:=\sum_{r=0}^{k} \frac{1}{r !} \int_{\langle u\rangle \leq|v| \leq 2\langle u\rangle}\left|f^{(r)}(u)\right| \frac{|v|^{r}}{\langle u\rangle}||\left|\chi_{x}\left(H^{\omega}-z\right)^{-1} \chi_{y}\right||| d u d v
$$

and

$$
I_{2}:=\frac{1}{k !} \int_{|v| \leq 2\langle u\rangle}\left|f^{(k+1)}(u)\right||v|^{k}||\left|\chi_{x}\left(H^{\omega}-z\right)^{-1} \chi_{y}\right| \| d u d v .
$$

As in [15], by (4.11) and $\left|\eta_{z}^{R L}\right|,\left|\eta_{z}\right| \geq|v|$, we have

$$
\begin{aligned}
& \||| \chi_{x}\left(H^{\omega}-z\right)^{-1} \chi_{y}|| \mid \\
& \leq \frac{C(d)}{|v|}\left\{1+\left(\left(|v|+|z|+\underset{\Lambda_{R L}}{\left.\left.\operatorname{ess} \sup _{-} \mathcal{V}_{-}^{\omega}+1\right)^{1 / 2} /|v|\right)}\right.\right.\right. \\
& \left.\quad \times\left(\left(|v|+|z|+\operatorname{ess} \sup \mathcal{V}_{-}^{\omega}+1\right)^{1 / 2} /|v|\right)^{(d-1) / 2}\right\} \\
& \quad \times \exp \left(-C \frac{|v||x-y|}{\sqrt{|v|+\operatorname{ess} \sup _{\Lambda_{R L}} \mathcal{V}_{-}+|z|}(\sqrt{|v|}+2)}\right) .
\end{aligned}
$$

If $\langle u\rangle \leq|v| \leq 2\langle u\rangle$, then we have $|v| \geq 1,|z| \leq \sqrt{2}|v|$ and

$$
\begin{aligned}
& ||\left|\chi_{x}\left(H^{\omega}-z\right)^{-1} \chi_{y}\right|||
\end{aligned}
$$

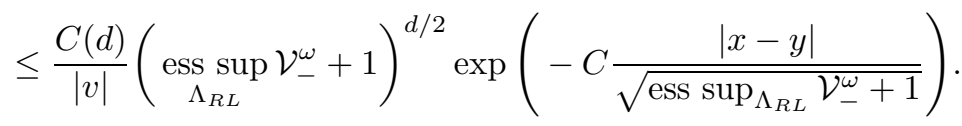


Therefore we have

$$
\begin{aligned}
& I_{1} \leq C(d)\left(\underset{\Lambda_{R L}}{\operatorname{ess} \sup _{-}} \mathcal{V}_{-}^{\omega}+1\right)^{d / 2}\left(\max _{0 \leq r \leq k} \frac{2^{r}}{r \cdot r !}\right)\|f\|_{(k)} \\
& \times \exp \left(-C \frac{|x-y|}{\sqrt{\operatorname{ess} \sup _{\Lambda_{R L}} \mathcal{V}_{-}^{\omega}+1}}\right) \text {. }
\end{aligned}
$$

If $|v| \leq 2\langle u\rangle$, then we have $|z| \leq 3\langle u\rangle$ and

$$
\begin{aligned}
& ||\left|\chi_{x}\left(H^{\omega}-z\right)^{-1} \chi_{y}\right|||
\end{aligned}
$$

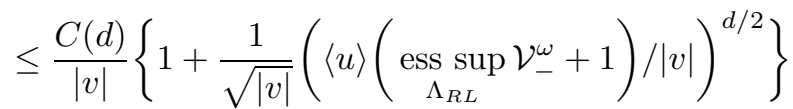

$$
\begin{aligned}
& \times \exp \left(-C \frac{|v||x-y|}{\langle u\rangle \sqrt{\operatorname{ess} \sup _{\Lambda_{R L}} \mathcal{V}_{-}^{\omega}+1}}\right) \text {. }
\end{aligned}
$$

Since

$$
\begin{aligned}
& \int_{0}^{2\langle u\rangle} v^{r} \exp \left(-C \frac{|v||x-y|}{\langle u\rangle \sqrt{\operatorname{ess} \sup _{\Lambda_{R L}} \mathcal{V}_{-}^{\omega}+1}}\right) \\
& \leq(2\langle u\rangle)^{r+1}\left(\frac{C r \sqrt{\operatorname{ess}^{\sup _{\Lambda_{R L}} \mathcal{V}_{-}^{\omega}+1}}}{\langle x-y\rangle}\right)^{r}
\end{aligned}
$$

for any $r \geq 1$, we have

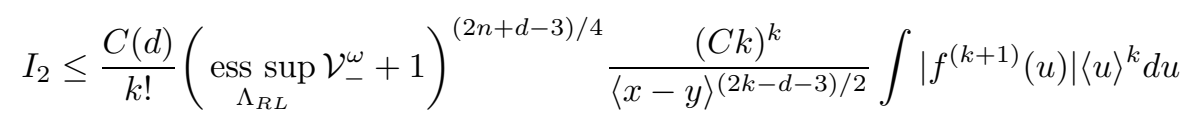

for any $k \geq(d+5) / 2$. Therefore we obtain $(4.9)$.

We should also modify Lemma 6.4 in [16] as follows:

Lemma 4.2. There exists a finite constant $C(d, h)$ satisfying the following: for any $\gamma>b d / h, \epsilon>0, a>0, E \in \widetilde{I}_{0}, 0<\varepsilon \leq 1,4 \leq L \in 2 \mathbb{N}$ and 
$0<b_{1}<b_{2}<b_{3} \leq 1$ such that $b_{2}-b_{1}>1 / 4$, we have

$$
\begin{aligned}
& P\left(\left\|\left|\left(\chi_{0, b_{3} L}-\chi_{0, b_{2} L}\right)\left(H_{L}^{\omega}-E-i \varepsilon\right)^{-1} \chi_{0, b_{1} L}\right|\right\|>a / 4,\right. \\
& \left.\quad \operatorname{ess} \sup _{\Lambda_{R L}} \mathcal{V}_{-}^{\omega} \leq(\log R L)^{(\epsilon+1) / \beta}\right) \\
& \leq \frac{L^{\gamma}}{a} E\left[\left\|\left(\chi_{0, b_{2} L}-\chi_{0, b_{2} L-1}\right)\left(H^{\omega}-E-i \varepsilon\right)^{-1} \chi_{0, b_{1} L}\right\| \mid\right. \\
& \left.\quad: \operatorname{ess} \sup \mathcal{V}_{-}^{\omega} \leq(\log R L)^{(\epsilon+1) / \beta}\right] \\
& \quad \Lambda_{R L} \\
& +Q_{\widetilde{I}_{0}} C(d, h)(1+|E|)^{h / 2}(\log R L)^{(\epsilon+1) h /(2 \beta)} / L^{\gamma h-b d}
\end{aligned}
$$

and

$$
\begin{aligned}
& P\left(E \notin \sigma\left(H_{L}^{\omega}\right),\left|\left\|\left|\left(\chi_{0, b_{3} L}-\chi_{0, b_{2} L}\right)\left(H_{L}^{\omega}-E\right)^{-1} \chi_{0, b_{1} L}\right|\right\|>a / 2,\right.\right. \\
& \left.\underset{\Lambda_{R L}}{\operatorname{ess} \sup } \mathcal{V}_{-}^{\omega} \leq(\log R L)^{(\epsilon+1) / \beta}\right) \\
& \leq \frac{L^{\gamma}}{a} E\left[\|\|\left(\chi_{0, b_{2} L}-\chi_{0, b_{2} L-1}\right)\left(H^{\omega}-E-i \varepsilon\right)^{-1} \chi_{0, b_{1} L} \mid \|\right. \\
& \left.: \underset{\Lambda_{R L}}{\operatorname{ess} \sup } \mathcal{V}_{-}^{\omega} \leq(\log R L)^{(\epsilon+1) / \beta}\right] \\
& +Q_{\widetilde{I_{0}}} C(d, h)(1+|E|)^{h / 2}(\log R L)^{(\epsilon+1) h /(2 \beta)} / L^{\gamma h-b d} \\
& +Q_{\widetilde{I}_{0}}(2 \sqrt{\varepsilon / a})^{h} L^{b d} \text {. }
\end{aligned}
$$

Proof. We take $\phi \in C_{0}^{\infty}\left(\Lambda_{L} \rightarrow[0,1]\right)$ so that $\phi=1$ on $\Lambda_{b_{1} L}, \phi=0$ on $\Lambda_{b_{2} L}^{c}, \operatorname{supp} \nabla \phi \subset \Lambda_{b_{2} L}-\overline{\Lambda_{b_{2} L-1}},|\nabla \phi| \leq 3 \sqrt{d}$ and $\left|\nabla^{2} \phi\right| \leq 7 d$. As in (4.11) and (4.12), we have

$$
\begin{aligned}
\phi & \left(H_{L}^{\omega}-E+i \varepsilon\right)^{-1}-\left(H^{\omega}-E+i \varepsilon\right)^{-1} \phi \\
= & 2\left(H^{\omega}-E+i \varepsilon\right)^{-1}(i \nabla \phi)(i \nabla+\mathcal{A})\left(H_{L}^{\omega}-E+i \varepsilon\right)^{-1} \\
& -\left(H_{L}^{\omega}-E+i \varepsilon\right)^{-1}(\Delta \phi)\left(H_{L}^{\omega}-E+i \varepsilon\right)^{-1}
\end{aligned}
$$

and

$$
\begin{aligned}
& \|\|\left(\chi_{0, b_{3} L}-\chi_{0, b_{2} L}\right)\left(H_{L}^{\omega}-E-i \varepsilon\right)^{-1} \chi_{0, b_{1} L} \mid \| \\
& \leq\left(2 \mid\left\|\left(\chi_{0, b_{3} L}-\chi_{0, b_{2} L}\right)\left(H^{\omega}-E-i \varepsilon\right)^{-1}(i \nabla+\mathcal{A})(\nabla \phi)\right\| \|\right. \\
& \left.\quad+\left\|||\left(\chi_{0, b_{3} L}-\chi_{0, b_{2} L}\right)\left(H_{L}^{\omega}-E-i \varepsilon\right)^{-1}(\Delta \phi) \mid\right\|\right) \\
& \quad \times\left\|\left|\left\|\left(\chi_{0, b_{2} L}-\chi_{0, b_{2} L-1}\right)\left(H^{\omega}-E-i \varepsilon\right)^{-1} \chi_{0, b_{1} L} \mid\right\| .\right.\right.
\end{aligned}
$$


As in (4.15), we have

$$
\begin{aligned}
& \left\|\left|(\nabla \phi)(i \nabla+\mathcal{A})\left(H_{L}^{\omega}-E+i \varepsilon\right)^{-1}\right|\right\| \\
& \leq \frac{C(d)}{\operatorname{dist}\left(\sigma\left(H_{L}^{\omega}\right), E\right)}\left(\operatorname{dist}\left(\sigma\left(H_{L}^{\omega}\right), E\right)+|E+i \varepsilon|+\underset{\Lambda_{L}}{\left.\operatorname{ess} \sup _{L} \mathcal{V}_{-}^{\omega}+1\right)^{1 / 2}} .\right.
\end{aligned}
$$

Therefore we have

$$
\begin{aligned}
& \|\|\left(\chi_{0, b_{3} L}-\chi_{0, b_{2} L}\right)\left(H_{L}^{\omega}-E-i \varepsilon\right)^{-1} \chi_{0, b_{1} L} \mid \| \\
& \leq \frac{C(d)}{\operatorname{dist}\left(\sigma\left(H_{L}^{\omega}\right), E\right)}\left(\operatorname{dist}\left(\sigma\left(H_{L}^{\omega}\right), E\right)+|E+i \varepsilon|+\underset{\Lambda_{L}}{\operatorname{ess} \sup } \mathcal{V}_{-}^{\omega}+1\right)^{1 / 2} \\
& \quad \times\left\|\mid\left(\chi_{0, b_{2} L}-\chi_{0, b_{2} L-1}\right)\left(H^{\omega}-E-i \varepsilon\right)^{-1} \chi_{0, b_{1} L}\right\|
\end{aligned}
$$

and the right hand side of (4.16) is dominated by

$$
\begin{aligned}
& P\left(\left\|\left|\left(\chi_{0, b_{2} L}-\chi_{0, b_{2} L-1}\right)\left(H^{\omega}-E-i \varepsilon\right)^{-1} \chi_{0, b_{1} L} \|\right| \mid>a / L^{\gamma},\right.\right. \\
& \left.\quad \quad \quad \Lambda_{R L} \sup _{-}^{\omega} \leq(\log R L)^{(\epsilon+1) / \beta}\right) \\
& +P\left(\operatorname{dist}\left(\sigma\left(H_{L}^{\omega}\right), E\right) \leq C(d)\left(|E+i \varepsilon|+(\log R L)^{(\epsilon+1) / \beta}+1\right)^{1 / 2} / L^{\gamma}\right) .
\end{aligned}
$$

Then, by the Chebyshev inequality and the Wegner estimate, we obtain (4.16).

If $E \notin \sigma\left(H_{L}^{\omega}\right)$, then

$$
\left(H_{L}^{\omega}-E\right)^{-1}=\left(H_{L}^{\omega}-E-i \varepsilon\right)^{-1}-i \varepsilon\left(H_{L}^{\omega}-E\right)^{-1}\left(H_{L}^{\omega}-E-i \varepsilon\right)^{-1}
$$

and

$$
\begin{aligned}
& \left\|\left|\left(\chi_{0, b_{3} L}-\chi_{0, b_{2} L}\right)\left(H_{L}^{\omega}-E\right)^{-1} \chi_{0, b_{1} L}\right|\right\| \\
& \leq\left\|\left|\left(\chi_{0, b_{3} L}-\chi_{0, b_{2} L}\right)\left(H_{L}^{\omega}-E-i \varepsilon\right)^{-1} \chi_{0, b_{1} L}\right|\right\|+\varepsilon / \operatorname{dist}\left(\sigma\left(H_{L}^{\omega}\right), E\right)^{2} .
\end{aligned}
$$

Therefore the right hand side of (4.18) is dominated by

$$
\begin{aligned}
& P\left(\left\|\left|\left(\chi_{0, b_{3} L}-\chi_{0, b_{2} L}\right)\left(H_{L}^{\omega}-E-i \varepsilon\right)^{-1} \chi_{0, b_{1} L}\|\mid\| a / 4,\right.\right.\right. \\
& \left.\quad \quad \quad \quad \Lambda_{R L} \sup _{-}^{\omega} \leq(\log R L)^{(\epsilon+1) / \beta}\right) \\
& +P\left(\operatorname{dist}\left(\sigma\left(H_{L}^{\omega}\right), E\right) \leq 2 \sqrt{\varepsilon / a}\right) .
\end{aligned}
$$

Then, by (4.16) and the Wegner estimate, we obtain (4.18). 
Proof of Theorem 4.1. Let $I$ be a compact interval included in $J$ and $\theta>b d / h$ specified later. For any $E \in I$ and $36<L \in 6 \mathbb{N}$, we set

$$
\begin{aligned}
P_{E, L}:= & P\left(E \in \sigma\left(H_{L}^{\omega}\right)\right) \\
& +P\left(E \notin \sigma\left(H_{L}^{\omega}\right),||\left|\Gamma_{0, L}\left(H_{L}^{\omega}-E\right)^{-1} \chi_{0, L / 3}\right| \|>1 /\left(2 L^{\theta}\right)\right) .
\end{aligned}
$$

If $E \notin \sigma\left(H_{L}^{\omega}\right)$, then, by using the resolvent identity and $\chi_{0, L}=\chi_{0,2 L / 3}+\left(\chi_{0, L}-\right.$ $\left.\chi_{0,2 L / 3}\right)$ as in [16], we have

$$
\begin{aligned}
& \left\||| \Gamma_{0, L}\left(H_{L}^{\omega}-E\right)^{-1} \chi_{0, L / 3}|\||\right. \\
& \leq\left\|\left|\Gamma_{0, L}\left(H_{L}^{\omega}-E-i \varepsilon\right)^{-1} \chi_{0, L / 3} \|\right|\right. \\
& \quad+\varepsilon\left|\left\|\Gamma_{0, L}\left(H_{L}^{\omega}-E\right)^{-1} \chi_{0,2 L / 3}\right\|\right| / \operatorname{dist}\left(\sigma\left(H_{L}^{\omega}\right), E\right) \\
& \quad+\varepsilon\left\|\left(\chi_{0, L}-\chi_{0,2 L / 3}\right)\left(H_{L}^{\omega}-E-i \varepsilon\right)^{-1} \chi_{0, L / 3}\right\||| \operatorname{dist}\left(\sigma\left(H_{L}^{\omega}\right), E\right) .
\end{aligned}
$$

Therefore we have

$$
P_{E, L} \leq \sum_{j=1}^{5} I_{j},
$$

where

$$
\begin{aligned}
& I_{1}:=P\left(||\left|\Gamma_{0, L}\left(H_{L}^{\omega}-E-i \varepsilon\right)^{-1} \chi_{0, L / 3}\right| \|>1 /\left(4 L^{\theta}\right),\right. \\
& \left.\underset{\Lambda_{R L}}{\operatorname{ess} \sup } \mathcal{V}_{-}^{\omega} \leq(\log R L)^{(\epsilon+1) / \beta}\right), \\
& I_{2}:=P\left(E \notin \sigma\left(H_{L}^{\omega}\right),||\left|\Gamma_{0, L}\left(H_{L}^{\omega}-E\right)^{-1} \chi_{0,2 L / 3}\right|||>1 / 2,\right. \\
& \left.\underset{\Lambda_{R L}}{\operatorname{ess} \sup } \mathcal{V}_{-}^{\omega} \leq(\log R L)^{(\epsilon+1) / \beta}\right), \\
& I_{3}:=P\left(\left\|\left|\left(\chi_{0, L}-\chi_{0,2 L / 3}\right)\left(H_{L}^{\omega}-E-i \varepsilon\right)^{-1} \chi_{0, L / 3} \|\right|>1 / 4,\right.\right. \\
& \text { ess } \left.\sup \mathcal{V}_{-}^{\omega} \leq(\log R L)^{(\epsilon+1) / \beta}\right), \\
& I_{4}:=P\left(\operatorname{dist}\left(\sigma\left(H_{L}^{\omega}\right), E\right) \leq 3 \varepsilon L^{\theta}\right), \\
& I_{5}:=P\left(\underset{\Lambda_{R L}}{\operatorname{ess} \sup } \mathcal{V}_{-}^{\omega}>(\log R L)^{(\epsilon+1) / \beta}\right)
\end{aligned}
$$

and $\epsilon>0$ is taken arbitrarily. By the assumptions (W) and (F), we have

$$
I_{4}:=Q_{\widetilde{I}_{0}}(3 \varepsilon)^{h} L^{\theta h+b d}
$$

and

$$
I_{5}:=\delta_{1} \exp \left(-\delta_{2}(\log R L)^{\epsilon}\right)
$$


By taking $a=L^{-\theta}, b_{1}=1 / 3, b_{2}=(L-3) / L$ and $b_{3}=(L-1) / L$ in Lemma 4.2, we have

$$
I_{1} \leq I_{1}^{\prime}+Q_{\widetilde{I}_{0}} C(I, d, h)(\log L)^{(\epsilon+1) h /(2 \beta)} / L^{\gamma h-b d},
$$

where

$$
\begin{gathered}
I_{1}^{\prime}:=L^{\gamma+\theta} E\left[\left\||| \Gamma_{0, L-2}\left(H^{\omega}-E-i \varepsilon\right)^{-1} \chi_{0, L / 3} \mid\right\|\right. \\
: \underset{\Lambda_{R L}}{\left.\operatorname{ess} \sup _{-} \leq(\log R L)^{(\epsilon+1) / \beta}\right]}
\end{gathered}
$$

and $\gamma>b d / h$ is specified later. This is estimated as

$$
I_{1}^{\prime} \leq I_{11}+I_{12}
$$

where

$$
I_{11}:=L^{\gamma+\theta} E\left[||\left|\Gamma_{0, L-2}\left(H^{\omega}-E-i \varepsilon\right)^{-1} f\left(H^{\omega}\right) \chi_{0, L / 3}\right|||\right]
$$

and

$$
\begin{aligned}
I_{12}:=L^{\gamma+\theta} E & {\left[\left\|\mid \Gamma_{0, L-2}\left(H^{\omega}-E-i \varepsilon\right)^{-1}\left(1-f\left(H^{\omega}\right)\right) \chi_{0, L / 3}\right\| \|\right.} \\
& \left.: \underset{\Lambda_{R L}}{\operatorname{ess} \sup _{-}} \mathcal{V}_{-}^{\omega} \leq(\log R L)^{(\epsilon+1) / \beta}\right] .
\end{aligned}
$$

As in [16], we have

$$
\begin{aligned}
& \left.I_{11} \leq L^{\gamma+\theta} \sum_{y \in \mathbb{Z}^{d} \cap \Lambda_{L / 3}} E\left[\left\||| \Gamma_{0, L-2}\left(H^{\omega}-E-i \varepsilon\right)^{-1} f\left(H^{\omega}\right) \chi_{y}\right\| \mid\right]\right] \\
& =L^{\gamma+\theta} \sum_{y \in \mathbb{Z}^{d} \cap \Lambda_{L / 3}} E\left[\|\left|\Gamma_{-y, L-2}\left(H^{\omega}-E-i \varepsilon\right)^{-1} f\left(H^{\omega}\right) \chi_{0}\right|||\right] \\
& \leq L^{\gamma+\theta}\left(\frac{L}{3}\right)^{d}\left(\frac{L}{3}-\frac{3}{2}\right)^{-n / 2} E\left[\left\|||\langle X\rangle^{n / 2}\left(H^{\omega}-E-i \varepsilon\right)^{-1} f\left(H^{\omega}\right) \chi_{0} \mid\right\|\right] \\
& \leq \frac{12^{n / 2}}{3^{d} L^{(n / 2)-d-\gamma-\theta}} \mathcal{O}_{\varepsilon}(n, f, E)^{1 / 2}
\end{aligned}
$$

where

$$
\mathcal{O}_{\varepsilon}(n, f, E):=E\left[\left\|||\langle X\rangle^{n / 2}\left(H^{\omega}-E-i \varepsilon\right)^{-1} f\left(H^{\omega}\right) \chi_{0}\right\| \|_{2}^{2}\right] .
$$

By using Proposition 4.4, we have

$$
I_{12} \leq C(c, f, k, I, \beta, \epsilon)(\log L)^{(\epsilon+1)(2 k+d-3) /(4 \beta)} / L^{k-1-\gamma-\theta-3 d / 2}
$$

for any $(d+5) / 2 \leq k \in \mathbb{N}$. 
$I_{2}$ and $I_{3}$ are also estimated similarly by using Lemma 4.2 appropriately. Therefore we obtain

$$
P_{E, L} \leq \sum_{j=1}^{3} P_{E, L}^{j}
$$

where

$$
\begin{aligned}
P_{E, L}^{1}:= & \frac{C(n, d)}{L^{(n / 2)-d-\gamma-\theta}} \mathcal{O}_{\varepsilon}(n, f, E)^{1 / 2} \\
P_{E, L}^{2}:= & C(d, f, k, I, \beta, \epsilon)(\log L)^{(\epsilon+1)(2 k+d-3) /(4 \beta)} / L^{k-1-\gamma-\theta-3 d / 2} \\
& +Q_{\widetilde{I}_{0}} C(I, d, h)(\log L)^{(\epsilon+1) h /(2 \beta)} / L^{\gamma h-b d} \\
& +\delta_{1} \exp \left(-\delta_{2}(\log R L)^{\epsilon}\right)
\end{aligned}
$$

and

$$
P_{E, L}^{3}:=Q_{\widetilde{I_{0}}}\left\{2^{h} \sqrt{\varepsilon^{h} L^{2 b d}}+(3 \varepsilon)^{h} L^{\theta h+b d}\right\} .
$$

For any $\varepsilon>0$ and $0<p_{0}<1$, we set

$$
L\left(\varepsilon, p_{0}\right):=\left[\left(\frac{p_{0}}{(3 \varepsilon)^{h}}\right)^{1 /(\theta h+b d)} \wedge\left(\frac{p_{0}}{\varepsilon^{h}}\right)^{1 /(2 b d)}\right]_{6 \mathbb{N}} .
$$

Then, since $(3 \varepsilon)^{h} L(\varepsilon)^{\theta h+b d}, \varepsilon^{h} L^{2 b d} \leq p_{0}$, we have

$$
P_{E, L\left(\varepsilon, p_{0}\right)}^{3} \leq Q_{\widetilde{I_{0}}}\left(2^{h} \sqrt{p_{0}}+p_{0}\right) .
$$

By Proposition 6.1 in [16], we have

$$
\mathcal{O}:=\varliminf_{\varepsilon \downarrow 0} \varepsilon^{1+\alpha} \int_{\mathbb{R}} \mathcal{O}_{\varepsilon}(n, f, E) d E<\infty .
$$

Then there exists a sequence $\left\{\varepsilon_{m}\right\}_{m}$ such that $\varepsilon_{m} \searrow 0$ as $m \rightarrow \infty, L\left(\varepsilon_{m}, p_{0}\right)>$ 36 and

$$
\varepsilon_{m}^{1+\alpha} \int_{\mathbb{R}} \mathcal{O}_{\varepsilon_{m}}(n, f, E) d E \leq 2 \mathcal{O}
$$

For any $m \in \mathbb{N}$ and $M>0$, we set

$$
A_{m . M}:=\left\{E \in I: \varepsilon_{m}^{1+\alpha} \mathcal{O}_{\varepsilon_{m}}(n, f, E) \leq M\right\} .
$$

Then we have $\left|I-A_{m, M}\right| \leq 2 \mathcal{O} / M$. Since

$$
\varepsilon_{m} \geq C(\theta, h, b, d)\left(\frac{p_{0}}{L\left(\varepsilon_{m}, p_{0}\right)^{\theta h+b d}}\right)^{1 / h},
$$


we have

$$
P_{E, L\left(\varepsilon_{m}, p_{0}\right)}^{1} \leq \frac{C(d, n, \theta, h, b) \sqrt{M}}{p_{0}^{(1+\alpha) /(2 h)} L\left(\varepsilon_{m}, p_{0}\right)^{(n / 2)-d-\gamma-\theta-(1+\alpha)(\theta h+b d) /(2 h)}}
$$

for any $E \in A_{m, M}$. Taking $v>0$ arbitrarily, we set $M(m):=2 \mathcal{O} L\left(\varepsilon_{m}, p_{0}\right)^{v}$.

For any $E^{\prime} \in I$, there exists $E \in A_{m, M(m)}$ such that $\left|E-E^{\prime}\right| \leq L\left(\varepsilon_{m}, p_{0}\right)^{-v}$. Then the quantity

$$
\begin{aligned}
& P_{E^{\prime}, L\left(\varepsilon_{m}, p_{0}\right)}^{\prime} \\
& :=P\left(E^{\prime} \in \sigma\left(H_{L\left(\varepsilon_{m}, p_{0}\right)}^{\omega}\right)\right) \\
& \quad+P\left(E^{\prime} \notin \sigma\left(H_{L\left(\varepsilon_{m}, p_{0}\right)}^{\omega}\right),\right. \\
& \left.\quad \quad\left\|\Gamma_{0, L\left(\varepsilon_{m}, p_{0}\right)}\left(H_{L\left(\varepsilon_{m}, p_{0}\right)}^{\omega}-E^{\prime}\right)^{-1} \chi_{0, L\left(\varepsilon_{m}, p_{0}\right) / 3}\right\|>L\left(\varepsilon_{m}, p_{0}\right)^{-\theta}\right)
\end{aligned}
$$

is dominated by

$$
P_{E, L\left(\varepsilon_{m}, p_{0}\right)}+P\left(\operatorname{dist}\left(E, \sigma\left(H_{L\left(\varepsilon_{m}, p_{0}\right)}^{\omega}\right)\right)<C L\left(\varepsilon_{m}, p_{0}\right)^{-(v-\theta) / 2}\right) .
$$

Therefore we obtain

$$
\begin{aligned}
& P_{E^{\prime}, L\left(\varepsilon_{m}, p_{0}\right)}^{\prime} \\
& \leq \frac{C(n, d, \theta, h, b) \sqrt{\mathcal{O}}}{p_{0}^{(1+\alpha) /(2 h)} L\left(\varepsilon_{m}, p_{0}\right)^{(n / 2)-d-\gamma-\theta-(1+\alpha)(\theta h+b d) /(2 h)-v / 2}} \\
& \quad+C(d, f, k, I, \beta, \epsilon)\left(\log L\left(\varepsilon_{m}, p_{0}\right)\right)^{(\epsilon+1)(2 k+d-3) /(4 \beta)} / L\left(\varepsilon_{m}, p_{0}\right)^{k-1-\gamma-\theta-3 d / 2} \\
& \quad+Q_{\widetilde{I_{0}}} C(d, h, I)\left(\log L\left(\varepsilon_{m}, p_{0}\right)\right)^{(\epsilon+1) h /(2 \beta)} / L\left(\varepsilon_{m}, p_{0}\right)^{\gamma h-b d} \\
& \quad+\delta_{1} \exp \left(-\delta_{2}\left(\log R L\left(\varepsilon_{m}, p_{0}\right)\right)^{\epsilon}\right) \\
& \quad+Q_{\widetilde{I}_{0}}\left(2^{h} \sqrt{p_{0}}+p_{0}\right) \\
& \quad+Q_{\widetilde{I_{0}}} C(h) / L\left(\varepsilon_{m}, p_{0}\right)^{h(v-\theta) / 2-b d} .
\end{aligned}
$$

If we can take $n \geq 0, \gamma, \theta>b d / h, v>0$ and $(d+5) / 2 \leq k \in \mathbb{N}$ so that

$$
\begin{aligned}
& n / 2>d+\gamma+\theta+(1+\alpha)(\theta h+b d) /(2 h)+v / 2, \\
& k>\gamma+\theta+3 d / 2-1, \\
& h v / 2>h \theta / 2+b d,
\end{aligned}
$$

then we have

$$
\varlimsup_{m \rightarrow \infty} P_{E^{\prime}, L\left(\varepsilon_{m}, p_{0}\right)}^{\prime} \leq Q_{\widetilde{I}_{0}}\left(2^{h} \sqrt{p_{0}}+p_{0}\right)
$$

and

$$
\varliminf_{L \rightarrow \infty} P\left(E^{\prime} \notin \sigma\left(H_{L}^{\omega}\right),||\left|\Gamma_{0, L}\left(H_{L}^{\omega}-E^{\prime}\right)^{-1} \chi_{0, L / 3}\right||| \leq L^{-\theta}\right)=1
$$


from which we have $E^{\prime} \in \Sigma_{M S A}$. For (4.18) to be satisfied, (4.4) is necessary and sufficient.

\section{§4.3. Proof of Proposition 4.1}

To prove Proposition 4.1, we use the following instead of Lemma A.4 in [16]:

Lemma 4.3. For $L>0$ and a compact set $B$ in $\mathbb{R}$, we set $\Theta^{\omega}:=$ $1-\left(\min B \wedge \operatorname{ess}_{\inf _{\Lambda_{2}}} \mathcal{V}^{\omega}\right)$. Then, for any $d / 2<m \in \mathbb{N}$, there exists a finite constant $C(d, m)$ such that

$$
\operatorname{Tr}\left[\chi_{0, L} E\left(B: H^{\omega}\right)\left(H^{\omega}+\Theta^{\omega}\right)^{-m} \chi_{0, L}\right] \leq C(d, m) L^{d} .
$$

Proof. We take $\left\{\phi_{j}\right\}_{j \in \mathbb{N}} \subset C_{0}^{\infty}\left(\Lambda_{2 L} \rightarrow[0,1]\right)$ so that $\phi_{1} \equiv 1$ on $\Lambda_{L}$ and $\phi_{j+1} \equiv 1$ on $\operatorname{supp} \phi_{j}$ for any $j \in \mathbb{N}$. Then we have

$$
\begin{aligned}
E & \left(B: H^{\omega}\right)\left(H^{\omega}+\Theta^{\omega}\right)^{-1} \phi_{j}=E\left(B: H^{\omega}\right) \phi_{j}\left(H_{2 L}^{\omega}+\Theta^{\omega}\right)^{-1} \\
& +E\left(B: H^{\omega}\right)\left(H^{\omega}+\Theta^{\omega}\right)^{-1}\left(i \nabla+\mathcal{A}_{\omega}\right)\left(2 i \nabla \phi_{j}\right)\left(H_{2 L}^{\omega}+\Theta^{\omega}\right)^{-1} \\
& +E\left(B: H^{\omega}\right)\left(H^{\omega}+\Theta^{\omega}\right)^{-1}\left(\Delta \phi_{j}\right)\left(H_{2 L}^{\omega}+\Theta^{\omega}\right)^{-1}
\end{aligned}
$$

for any $j \in \mathbb{N}$. By this, we have

$$
\begin{aligned}
& \chi_{0, L} E\left(B: H^{\omega}\right)\left(H^{\omega}+\Theta^{\omega}\right)^{-m} \chi_{0, L} \\
& =\chi_{0, L} E\left(B: H^{\omega}\right)\left(H^{\omega}+\Theta^{\omega}\right)^{-m+1} \phi_{1}\left(H_{2 L}^{\omega}+\Theta^{\omega}\right)^{-1} \chi_{0, L} \\
& \quad+\chi_{0, L} E\left(B: H^{\omega}\right)\left(H^{\omega}+\Theta^{\omega}\right)^{-m}\left(i \nabla+\mathcal{A}_{\omega}\right)\left(2 i \nabla \phi_{1}\right) \\
& \quad \times\left(H_{2 L}^{\omega}+\Theta^{\omega}\right)^{-1} \chi_{0, L} \\
& \quad+\chi_{0, L} E\left(B: H^{\omega}\right)\left(H^{\omega}+\Theta^{\omega}\right)^{-m}\left(\Delta \phi_{1}\right)\left(H_{2 L}^{\omega}+\Theta^{\omega}\right)^{-1} \chi_{0, L} .
\end{aligned}
$$

By using (4.19) again, the third term is rewritten as

$$
\begin{aligned}
& \chi_{0, L} E\left(B: H^{\omega}\right)\left(H^{\omega}+\Theta^{\omega}\right)^{-m} \phi_{2}\left(H_{2 L}^{\omega}+\Theta^{\omega}\right)^{-1}\left(\Delta \phi_{1}\right)\left(H_{2 L}^{\omega}+\Theta^{\omega}\right)^{-1} \chi_{0, L} \\
& +\chi_{0, L} E\left(B: H^{\omega}\right)\left(H^{\omega}+\Theta^{\omega}\right)^{-m}\left(i \nabla+\mathcal{A}_{\omega}\right)\left(2 i \nabla \phi_{2}\right)\left(H_{2 L}^{\omega}+\Theta^{\omega}\right)^{-1} \\
& \quad \times\left(\Delta \phi_{1}\right)\left(H_{2 L}^{\omega}+\Theta^{\omega}\right)^{-1} \chi_{0, L} \\
& +\chi_{0, L} E\left(B: H^{\omega}\right)\left(H^{\omega}+\Theta^{\omega}\right)^{-m}\left(\Delta \phi_{2}\right)\left(H_{2 L}^{\omega}+\Theta^{\omega}\right)^{-1} \\
& \quad \times\left(\Delta \phi_{1}\right)\left(H_{2 L}^{\omega}+\Theta^{\omega}\right)^{-1} \chi_{0, L} .
\end{aligned}
$$

We repeat this calculation also for the other terms of the right hand side of (4.20). 
By the Feynman-Kac-Itô formula and the diamagnetic inequality, we have

$$
\begin{gathered}
\operatorname{Tr}\left[\exp \left(-t\left(H_{2 L}^{\omega}+\Theta^{\omega}\right)\right)\right] \leq e^{-t} \operatorname{Tr}\left[\exp \left(t \Delta_{2 L}\right)\right] \\
\leq C(d) L^{d} e^{-t} / t^{d / 2}
\end{gathered}
$$

where $\exp \left(-t\left(H_{2 L}^{\omega}+\Theta^{\omega}\right)\right)$ and $\exp \left(t \Delta_{2 L}\right)$ are the heat semigroups generated by $H_{2 L}^{\omega}+\Theta^{\omega}$ and the negative Dirichlet Laplacian $-\Delta_{2 L}$ on $\Lambda_{2 L}$, respectively (cf. [3]). Thus, for any $p>d$, we have

$$
\|\left(H_{2 L}^{\omega}+\Theta^{\omega}\right)^{-1 / 2}||_{p}^{p} \leq C(d, p) L^{d} .
$$

By applying this and

$$
\|\left|\left(i \nabla+\mathcal{A}_{\omega}\right)\left(H_{2 L}^{\omega}+\Theta^{\omega}\right)^{-1 / 2}\right||| \leq 1
$$

to the expansion of $\chi_{0, L} E\left(B: H^{\omega}\right)\left(H^{\omega}+\Theta^{\omega}\right)^{-m} \chi_{0, L}$, we have

$$
\begin{aligned}
& \operatorname{Tr}\left[\chi_{0, L} E\left(B: H^{\omega}\right)\left(H^{\omega}+\Theta^{\omega}\right)^{-m} \chi_{0, L}\right] \\
& \leq C^{\prime}(d, m)\left|\left\|\left(H_{2 L}^{\omega}+\Theta^{\omega}\right)^{-1 / 2} \mid\right\|_{2 m}^{2 m} \leq C(d, m) L^{d}\right.
\end{aligned}
$$

for $m>d / 2$.

Proof of Proposition 4.1. We can prove (4.7) and the first and the second inequalities in (4.5) and (4.6) by the same argument as in [16].

For the third inequalities of (4.5) and (4.6), we estimate as

$$
M_{\omega}(n, f, t) \leq C(d, n) \sum_{a \in \mathbb{Z}^{d}}\langle a\rangle^{n}\left|\left\|\chi_{a} \mathcal{Y}\left(H^{\omega}\right) \chi_{0} \mid\right\| \times\|\| \chi_{0} \mathcal{Y}\left(H^{\omega}\right) \chi_{a} \|_{1}\right.
$$

as in [16], where $\mathcal{Y}(u)=e^{-i t u} f(u)$ for $u \in \mathbb{R}$. By Proposition 4.4, we have

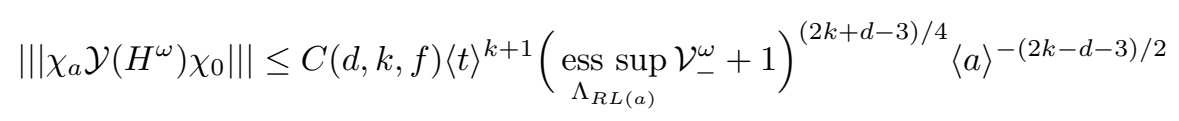

for any $(d+5) / 2 \leq k \in \mathbb{N}$, where $L(a)=2|a|+1$. On the other hand, we have

$$
\begin{aligned}
& \left\||| \chi_{0} \mathcal{Y}\left(H^{\omega}\right) \chi_{a} \mid\right\|_{1} \leq 4 \operatorname{Tr}\left[\chi_{0, L(a)}\left|\mathcal{Y}\left(H^{\omega}\right)\right| \chi_{0, L(a)}\right] \\
& \leq C(f, m)\left|\Theta^{\omega}\right|^{[[d / 2]]} \operatorname{Tr}\left[\chi_{0, L(a)} E\left(\operatorname{supp} f: H^{\omega}\right)\left(H^{\omega}+\Theta^{\omega}\right)^{-[[d / 2]]} \chi_{0, L(a)}\right],
\end{aligned}
$$

where $\Theta^{\omega}:=1-\left(\min \operatorname{supp} f \wedge \operatorname{ess}_{\inf _{\Lambda_{2 L(a)}}} \mathcal{V}^{\omega}\right)$. Then we have

$$
\left\|\left|\chi_{0} \mathcal{Y}\left(H^{\omega}\right) \chi_{a}\right|\right\|_{1} \leq C(f, d)\left(\underset{\Lambda_{2 L(a)}}{\operatorname{ess} \sup _{-}} \mathcal{V}_{-1}^{[[d / 2]]}\langle a\rangle^{d}\right.
$$


by Lemma 4.3. Therefore we obtain

$$
\begin{aligned}
M_{\omega}(n, f, t) \leq & C(d, n, k, f)\langle t\rangle^{k+1} \sum_{a \in \mathbb{Z}^{d}}\langle a\rangle^{(2 n+3 d+3-2 k) / 2} \\
& \times\left(\operatorname{coss}_{\Lambda_{R L(a)}}^{\operatorname{ess} \mathcal{V}_{-}^{\omega}+1}\right)^{(4[[d / 2]]+2 k+d-3) / 4} .
\end{aligned}
$$

For (4.5), we use Lemma 3.2 (ii) to obtain

$$
\underset{\Lambda_{R L(a)}}{\operatorname{ess} \sup _{-}} \mathcal{V}_{-}^{\omega}+1 \leq C(\beta, \omega)(\log \langle a\rangle+1)^{2 / \beta} .
$$

Then we have

$$
\begin{aligned}
M_{\omega}(n, f, t) \leq & C(d, n, k, f, \beta, \omega)\langle t\rangle^{k+1} \sum_{a \in \mathbb{Z}^{d}}\langle a\rangle^{(2 n+3 d+3-2 k) / 2} \\
& \times(\log \langle a\rangle+1)^{(4[[d / 2]]+2 k+d-3) /(2 \beta)}
\end{aligned}
$$

The right hand side is finite if $k>(2 n+5 d+3) / 2$. Therefore, by taking $k=[[n+(5 d+1) / 2]]+1$, we obtain $(4.5)$.

For (4.6), we use Lemma 3.2 (i) to obtain

$$
\begin{aligned}
& E\left[\left(\begin{array}{l}
\left.\operatorname{ess} \sup _{\Lambda_{R L(a)}} \mathcal{V}_{-}^{\omega}+1\right) \\
\leq
\end{array}\right)^{(4[[d / 2]]+2 k+d-3) / 4}\right] \\
& \leq C(d, k, \beta)(\log \langle a\rangle+1)^{(4[[d / 2]]+2 k+d-3) /(4 \beta)} .
\end{aligned}
$$

Then we have

$$
\begin{aligned}
\mathbb{M}(n, f, t) \leq & C(d, n, k, f, \beta)\langle t\rangle^{k+1} \sum_{a \in \mathbb{Z}^{d}}\langle a\rangle^{(2 n+d+3-2 k) / 2} \\
& \times(\log \langle a\rangle+1)^{(4[[d / 2]]+2 k+d-3) /(4 \beta)} .
\end{aligned}
$$

Therefore, by taking $k=[[n+(5 d+1) / 2]]+1$, we obtain $(4.6)$.

\section{References}

[1] Barbaroux, J.-M., Combes, J. M. and Hislop, P. D., Localization near band edges for random Schrödinger operators, Helv. Phys. Acta, 70 (1997), 16-43.

[2] Birman, M. S. and Yafaev, D. R., The spectral shift function. The work of M. G. Krein and its further development, St. Petersburg Math. J., 4 (1992), 833-870.

[3] Broderix, K., Hundertmark, D. and Leschke, H., Continuity properties of Schrödinger semigroups with magnetic fields, Rev. Math. Phys., 12 (2000), 181-225. 
[4] Cycon, H. L., Froese, R. G., Kirsch, W. and Simon, B., Schrödinger operators, Springer, Berlin-Heidelberg-New York, 1987.

[5] Combes, J. M. and Hislop, P. D., Localization for some continuous, random Hamiltonians in $d$-dimensions, J. Funct. Anal., 124 (1994), 149-180.

[6] Combes, J. M., Hislop, P. D., Klopp, F. and Nakamura, S., The Wegner estimate and the integrated density of states for some random operators, Proc. Indian Acad. Sci. Math. Sci., 112 (2002), 31-53.

[7] Combes, J. M., Hislop, P. D. and Nakamura, S., The $L^{p}$-theory of the spectral shift function, the Wegner estimate, and the integrated density of states for some random operators, Comm. Math. Phys., 218 (2001), 113-130.

[8] Davies, E. B., Spectral theory and differential operators, Cambridge University Press, Cambridge, 1995.

[9] Doob, J. L., Stochastic processes, Wiley, New York, 1953.

[10] Fernique, X., Regularité des trajectoires des fonctions aléatoires gaussiennes, In: Hennequin, P.-L., (ed.), École d'Été de Probabilités de Saint-Flour, IV-1974, Lecture Notes in Math., 480 (Springer, Berlin, 1975), 1-96.

[11] Fischer, W., Hupfer, T., Leschke, H. and Müller, P., Existence of the density of states for multi-dimensional continuum Schrödinger operators with Gaussian random potentials, Comm. Math. Phys., 190 (1997), 133-141.

[12] Fischer, W., Leschke, H. and Müller, P., Spectral localization by Gaussian random potentials in multi-dimensional continuous space, J. Statist. Phys., 101 (2000), 935985.

[13] Fröhlich, J. and Spencer, T., Absence of diffusion in the Anderson tight binding model for large disorder or low energy, Comm. Math. Phys., 88 (1983), 151-184.

[14] Germinet, F. and Klein, A., Bootstrap multiscale analysis and localization in random media, Comm. Math. Phys., 222 (2001), 415-448.

[15] Germinet, F. and Klein, A., Operator kernel estimates for functions of generalized Schrödinger operators, Proc. Amer. Math. Soc., 131 (2003), 911-920.

[16] , A characterization of the Anderson metal-insulator transport transition, Preprint.

[17] Explicit finite volume criteria for localization in continuous random media and applications, Preprint.

[18]

[19] Gilbarg, D. and Trudinger, N. S., Elliptic partial differential equations of second order, Second edition, Springer, Berlin, 1983.

[20] Hislop, P. D. and Klopp, F., The integrated density of states for some random operators with non-sign definite potentials, J. Funct. Anal., 195 (2002), 12-47.

[21] Hupfer, T., Leschke, H., Müller, P. and Warzel, S., The absolute continuity of the integrated density of states for magnetic Schrödinger operators with certain unbounded random potentials, Comm. Math. Phys., 221 (2001), 229-254.

[22] Klopp, F., Localization for some continuous random Schrödinger operators, Comm. Math. Phys., 167 (1995), 553-569.

[23] Klopp, F., Nakamura, S., Nakano, F. and Nomura, Y., Anderson localization for 2D discrete Schrödinger operator with random vector potential, Preprint.

[24] Kirsch, W., Wegner estimates and Anderson localization for alloy-type potentials, Math. Z., 221 (1996), 507-512.

[25] Kirsch, W., Stollmann, P. and Stolz, G., Anderson localization for random Schrödinger operators with long range interactions, Comm. Math. Phys., 195 (1998), 495-507.

[26] Kirsch, W. and Veselić, I., Wegner estimate for sparse and other generalized alloy type potentials, Proc. Indian Acad. Sci. (Math. Sci.), 112 (2002), 131-146.

[27] Kotani, S. and Simon, B., Localization in general one-dimensional random systems. II. continuous Schrödinger operators, Comm. Math. Phys., 112 (1987), 103-119.

[28] Kurata, K., Local boundedness and continuity for weak solutions of $-(\nabla-i b)^{2} u+V u=$ 0, Math. Z., 224 (1997), 641-653. 
[29] Leinfelder, H. and Simader, C. G., Schrödinger operators with singular magnetic vector potentials, Math. Ann., 294 (1992), 195-221.

[30] Nualart, D., The Malliavin calculus and related topics, Springer, New York, 1995.

[31] Pastur, L. and Figotin, A., Spectra of random and almost-periodic operators, Springer, Berlin, 1992.

[32] Reed, M. and Simon, B., Methods of modern mathematical physics II Fourier analysis, self-adjointness, Academic Press, New York, 1975.

[33] Simon, B., Trace ideals and their applications, Cambridge University Press, CambridgeNew York, 1979.

[34] _ Functional integration and quantum physics, Academic Press, New YorkLondon, 1979.

[35] , Spectral averaging and the Krein spectral shift, Proc. Amer. Math. Soc., 126 (1998), 1409-1413.

[36] Stollmann, P., Wegner estimates and localization for continuum Anderson models with some singular distributions, Arch. Math., 75 (2000), 307-311.

[37] _ Caught by disorder, Bound states in random media, Birkhäuser, Boston, 2001.

[38] Ueki, N., On spectra of random Schrödinger operators with magnetic fields, Osaka J. Math., 31 (1994), 177-187.

[39] Veselić, I., Wegner estimate and the density of states of some indefinite alloy-type Schrödinger operators, Lett. Math. Phys., 59 (2002), 199-214.

[40] Wegner, F., Bounds on the density of states in disordered systems, Z. Phys. B, 44 (1981), 9-15.

[41] Yafaev, D. R., Mathematical scattering theory, general theory, American Mathematical Society, Providence, RI, 1992. 